Método do averaging para sistemas de Filippov 

SERVIÇO DE PÓS-GRADUAÇÃO DO ICMC-USP

Data de Depósito: 26/02/2015

Assinatura:

\title{
Método do averaging para sistemas de Filippov
}

\author{
Camila Aparecida Benedito Rodrigues ${ }^{1}$
}

Orientadora: Profa. Dra. Regilene Delazari dos Santos Oliveira

Dissertação apresentada ao Instituto de Ciências Matemáticas e de Computação - ICMC-USP, como parte dos requisitos para obtenção do título de Mestre em Ciências - Matemática. VERSÃO REVISADA

USP - São Carlos

Fevereiro de 2015

${ }^{1}$ Suporte financeiro da FAPESP. Processo no 2012/22000-0 
Ficha catalográfica elaborada pela Biblioteca Prof. Achille Bassi e Seção Técnica de Informática, ICMC/USP, com os dados fornecidos pelo(a) autor(a)

Rodrigues, Camila Aparecida Benedito
Método do averaging para sistemas de Filippov /
Camila Aparecida Benedito Rodrigues; orientadora
Regilene Delazari dos Santos Oliveira. -- São
Carlos, 2015.
102 p.
Dissertação (Mestrado - Programa de Pós-Graduação
em Matemática) -- Instituto de Ciências Matemáticas
e de Computação, Universidade de São Paulo, 2015.
1. averaging. 2. órbitas periódicas. 3. sistemas
de Filippov. Iliveira, Regilene Delazari dos
Santos, orient. II. Título.


"Matemático não inverte os papéis, eleva a -1; Matemático não desmente o outro, reduz ao absurdo; Matemático não facilita as coisas, faz mudança de base; Matemático não segue preceitos, parte dos axiomas; Matemático não releva, usa o elemento neutro; Matemático não é bipolar, se comporta como a função modular;

Matemático não se muda, translada; Matemático não faz muitas coisas ao mesmo tempo, usa regra da cadeia; Matemático não mede as consequências, pensa nos corolários;

Matemático não tá em pé e deita, faz a transposta; Matemático não peca, divide por zero."

Autor Desconhecido 


\section{Agradecimentos}

Gostaria de agradecer primeiramente a Deus e a Nossa Senhora Aparecida, pois sem seus auxílios nada disso seria possível.

Agradecer o imensurável amor de meus pais, Valentina e João, e de toda a minha família que sempre me apoiou nos momentos difíceis e me deu forças para seguir em frente com meu objetivo. Eles sempre se fizeram presentes em minha jornada.

Tenho tanta sorte que Deus me proporcionou uma segunda família, a qual me socorreu igualmente quando necessário: um agradecimento à Rosângela e Sebastião, meus pais aqui em São Carlos. Dessa família veio meu namorado Leonardo, a quem devo um obrigado sem tamanho. Quando nos momentos de desespero e nas madrugadas em claro pensei em desistir de tudo, ele me ajudou com palavras de carinho e confiança... "Tudo vai dar certo, eu confio em você!". Agradeço também pelas vezes que me empolguei falando de minhas ferramentas matemáticas e mesmo sem ser de minha área, me deu total atenção.

Não posso deixar de lembrar os amigos que fiz ao longo desses seis anos aqui na Usp entre graduação e mestrado, em especial: Matheus e Alex, sem a ajuda de vocês com o Latex, não teria conseguido sozinha; Heloísa, minha colega de quarto por três anos, que tornou-se mais que uma irmã para mim ; Liliam e Rafael, que sempre podia contar nessa dura jornada; Jean, a quem recorria com dúvidas de análise. Obrigada a todos pela amizade e carinho.

Aos muitos professores que tive durante toda a vida, em especial aos Professores Antônio Poli e Edna, de Boa Esperança do Sul. Ele me fez gostar de ser desafiada em matemática e Professora Edna me fez entender e gostar cada vez mais da Língua 
Portuguesa e Literatura, com suas fantásticas aulas no ensino médio.

A Professora Sandra Godoy do ICMC, que me direcionou para a área que estou hoje, quando me senti perdida nos últimos minutos.

A Professora Ana Mereu, da UFSCar, que se dispôs a trabalhar comigo e com Regilene desde o ínicio do projeto e ao Professor Jaume Llibre, da Universidade Autônoma de Barcelona que me deu o privilégio de trabalhar com ele durante uma visita ao ICMCUSP.

Agradeço a Fundação de Amparo à Pesquisa do Estado de São Paulo (FAPESP) pelo suporte financeiro.

Por fim, mas não menos importante, agradeço à minha orientadora Regilene Oliveira, por toda atenção, disposição e porque não, coragem para orientar uma aluna que mudou de área no último semestre da graduação. Obrigada pela confiança Regilene, você me mostrou como unir análise, geometria, álgebra e o computador e fazer toda essa mistura dar certo. 


\section{Resumo}

Um dos mais investigados problemas na teoria qualitativa dos sistemas dinâmicos no plano é o XVI problema de Hilbert que investiga uma cota superior para o número de ciclos limites em sistemas diferenciais polinomiais e suas posições relativas. Por outro lado, os sistemas diferenciais suaves por partes tem despertado o interesse de muitos pesquisadores recentemente devido a sua estreita relação com outras áreas das ciências como física, biologia, economia e engenharias. Portanto é natural a busca pela extensão das técnicas e ferramentas da teoria qualitativa para essa classe de sistemas. Nessa dissertação apresentamos uma generalização da técnica do averaging para uma classe especial dos sistemas de Filippov, conhecida como sistemas diferenciais contínuos por partes, desenvolvida por Llibre-Novaes-Teixeira e, aplicamos essa técnica na investigação de uma classe particular de sistemas, que chamamos do tipo Kukles generalizado.

Palavras-chave: XVI problema de Hilbert, método do averaging, sistemas de Filippov. 


\section{Abstract}

One of the most investigated problems in the qualitative theory of dynamical systems in the plane is the XVI Hilbert's problem which asks for the maximum number and position of limit cycles for all planar polynomial differential systems of degree $n$. On the other hand, recently piecewise continuous differential systems have attracting the interest of many researches specially because of their close relation with other sciences for instance physics, biology, economy and engineering. These relations motivate extensions of the qualitative tools for this class of systems. In this work we present a generalization of the averaging theory for a class of Filippov systems, namely piecewise continuous differential systems, developed by Llibre-Novaes-Teixeira and, we apply this theory to a particular class of differential systems, which we nominate generalized Kukles type.

Key words: XVI Hilbert's problem, averaging method, Filippov's systems. 


\section{Sumário}

Introdução

1 Aspectos gerais 5

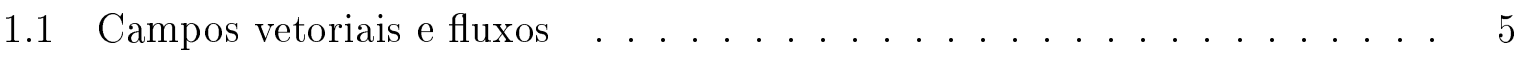

1.2 Retrato de fase de um sistema diferencial . . . . . . . . . . . . . . 7

1.3 Ciclos limites . . . . . . . . . . . . . . . . . . 8

1.3.1 O XVI problema de Hilbert . . . . . . . . . . . . . . . 9

1.3.2 A versão fraca do XVI problema de Hilbert . . . . . . . . . . . . 11

1.4 Integrais Abelianas . . . . . . . . . . . . . . . . . . . . . . 14

1.5 O método do averaging para sistemas diferenciais contínuos . . . . . . . . . 20

1.5.1 Averaging analítico . . . . . . . . . . . . . . . 20

1.5.2 Averaging via grau de Brouwer ... . . . . . . . . . 22

1.5.3 Averaging períodico de segunda ordem via grau de Brouwer . . . . 33

2 Sistemas de Filippov $\quad 35$

2.1 Trajetória local e singularidades . . . . . . . . . . . . . . 36

2.2 Separatrizes, órbitas periódicas e ciclos . . . . . . . . . . . . . . . 44

2.3 Equivalência topológica . . . . . . . . . . . . . . . . . . . . . . . 49

$3 \quad$ O averaging para sistemas diferenciais suaves por partes $\quad 51$

3.1 Soluções de Filippov . . . . . . . . . . . . . . . . . . . . . . . . . 51

3.2 O grau de Brouwer sobre outro ponto de vista . . . . . . . . . . . 55 
3.3 A técnica do averaging . . . . . . . . . . . . . . 56

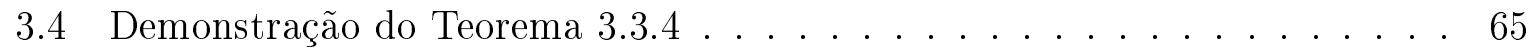

4 Aplicações do averaging para alguns sistemas suaves por partes $\quad 73$

4.1 Ciclos limites que bifurcam de um centro isócrono específico . . . . . . . . 74

4.2 Sistemas diferenciais suaves por partes do tipo Kukles . . . . . . . . . . . . 79

4.2.1 Exemplos ............................ 93

4.2 .2 Conjectura . . . . . . . . . . . . . . . 96

$\begin{array}{lll}5 & \text { Considerações finais } & 97\end{array}$ 


\section{Introdução}

Em 1900, durante o Congresso Internacional de Matemática ocorrido em Paris, David Hilbert $[17,18]$ propôs uma lista com vinte e três problemas matemáticos para serem resolvidos no século XX. Dentre eles, destacamos o décimo sexto. Tal problema é dividido em duas partes, sendo a primeira delas de interesse da geometria algébrica e a segunda parte consiste em determinar qual o número máximo de ciclos limites que um sistema diferencial polinomial planar de grau $n$ pode ter e qual a posição relativa desses ciclos.

A resposta para esse problema ainda não foi alcançada. Écalle [13] e Il'Yashenko [19] mostraram que o número de ciclos limites de um sistema diferencial polinomial planar, usualmente denotado por $H(n)$, é finito (a prova dessa afirmação não é acessível até mesmo para renomados matemáticos).

Dado que a questão inicial se tornou muito difícil de ser investigada, novos enunciados foram dados ao problema. Talvez a versão mais investigada atualmente seja a versão fraca do XVI problema, proposta por Arnol'd [3, 5]. A versão propõe a investigação do número máximo de ciclos limites que bifurcam de um sistema diferencial polinomial planar que possui uma singularidade do tipo centro.

Visando resolver esse problema, muitas técnicas foram desenvolvidas, a maioria delas baseadas na aplicação de primeiro retorno de Poincaré. Dentre elas podemos citar o método da integral de Poincaré-Melnikov, o método das integrais abelianas e a teoria da média ou averaging. Este último foi desenvolvido inicialmente como uma ferramenta analítica, afim de aproximar soluções de dois sistemas diferenciais (ver [35]). O método exibe uma relação quantitativa entre as soluções de um sistema diferencial não autônomo 
e as soluções de sistema diferencial autônomo. Juntamente com o Teorema da Função Implícita, a teoria do averaging passou a ser empregada para detectar a existência de soluções periódicas do sistema autônomo. Os resultados apresentados nessa dissertação seguem nesse sentido.

Detalhes analíticos sobre a teoria do averaging para sistemas diferenciáveis podem ser encontrado em [31]. Em [38], Verhulst apresenta o teorema do averaging sendo usado para encontrar uma estimativa para o número de órbitas periódicas em sistemas diferenciais do tipo $\dot{x}=\varepsilon f^{1}(t, x)+\varepsilon^{2} f^{2}(t, x, \varepsilon)$. O teorema exigia, entre outras hipóteses, que $f^{1}, f^{2}, D_{x} f^{1}, D_{x}^{2} f^{1}$ e $D_{x} f^{2}$ fossem funções bem definidas, contínuas e limitadas por uma constante $M$, independente de $\varepsilon$ em $[0, \infty) \times D,-\varepsilon_{f}<\varepsilon<\varepsilon_{f}$. Em [11], Buica e Llibre demonstraram o mesmo resultado usando o grau de Brouwer e enfraquecendo as hipóteses (as condições citadas acima foram substituídas por $f^{1}$ e $f^{2}$ serem localmente Lipschitz com respeito à variável $x$ ). Existem trabalhos recentes, como o de Llibre, Novaes e Teixeira [27], que generalizam o método, garantindo que sob certas condições ele pode ser aplicado a sistemas diferenciais contínuos do tipo $\dot{x}=f^{0}(x, t)+\varepsilon f^{1}(x, t)+\varepsilon^{2} f^{2}(x, t, \varepsilon)$.

Tudo o que foi descrito até aqui são estudos feitos para sistemas diferenciais contínuos. O objetivo desse trabalho é descrever o uso da teoria do averaging para sistemas diferenciais descontínuos, conhecidos como sistemas de Filippov.

O estudo de sistemas diferenciais suaves por partes tem se desenvolvido muito rápido e despertado o interesse de muitos pesquisadores ultimamente. Este fato se deve principalmente por sua forte relação com outras áreas das ciências como física, biologia [8], economia [16, 20] e engenharias. Certos fenômenos em sistemas de controle [7], impacto e fricção em sistemas mecânicos [9], e oscilações não-lineares [2] são as principais motivações para o estudo e entendimento da dinâmica de tais sistemas. Além das aplicações, a compreensão matemática de tais sistemas é por si só um incentivo para sua análise.

Entretanto existem poucas ferramentas analíticas para o estudo qualitativo de tais sistemas. Atualmente, a teoria do averaging para sistemas suaves por partes, desenvolvida por Llibre, Novaes e Teixeira em [28] provavelmente é uma das técnicas mais investigadas e empregadas no estudo da existência de ciclos limites e órbitas periódicas para essa família de sistemas. A técnica é uma extensão da teoria do averaging para sistemas suaves e 
nos permite relacionar o número de órbitas periódicas de um sistema de Filippov com o número de zeros de uma determinada função, que será chamada nessa dissertação de função promediada.

Essa dissertação está organizada da seguinte forma. No primeiro capítulo, apresentamos uma breve introdução dos conceitos necessários para o estudo proposto, bem como uma análise do método das integrais abelianas e do averaging para sistemas diferenciais contínuos. Em seguida, no Capítulo 2, definimos sistemas de Filippov e discutimos suas propriedades. No Capítulo 3 estudamos o que chamamos de averaging descontínuo, onde apresentamos a demonstração do teorema apresentado em [28]. No quarto capítulo dessa dissertação apresentamos duas aplicações da teoria do averaging descontínuo.

A primeira aplicação apresentada foi desenvolvida por Llibre-Mereu [25] para investigação dos sistemas com centros isócronos de uma dada forma normal. A segunda aplicação é original e foi desenvolvida com base no trabalhos de Llibre-Mereu ([23], [24]). Em ambos trabalhos os autores investigam sobre o número de ciclos limites que bifurcam de um centro linear quando perturbado dentro de classes especificas de sistemas diferenciais planares, em [23] na classe dos sistemas diferenciais planares do tipo Kukles generalizado e em [24], quando o sistema é perturbado na classe dos sistemas suaves por partes do tipo Lienard com $n$ retas de descontinuidades. Nessa dissertação, investigamos o número de ciclos limites que bifurcam de um centro linear quando perturbado na classe dos sistemas por partes do tipo Kukles generalizado, que é uma classe dos sistemas diferenciais descontínuos possuindo $l$ retas de descontinuidades passando pela origem dada por

$$
\dot{X}= \begin{cases}X_{1}(x, y) & \text { se } h(x, y)>0 \\ X_{2}(x, y) & \text { se } h(x, y)<0\end{cases}
$$

onde $X_{j}(x, y)=\left(\begin{array}{c}y, \\ -x-\varepsilon\left(f_{n_{1}}^{j}(x)+g_{n_{2}}^{j}(x) y+h_{n_{3}}^{j}(x) y^{2}+d_{0}^{j} y^{3}\right),\end{array}\right) \operatorname{com} f_{n_{1}}^{j}(x), g_{n_{2}}^{j}(x) \mathrm{e}$ $h_{n_{3}}^{j}(x)$ polinômios de grau $n_{1}, n_{2}$ e $n_{3}$ respectivamente, e $d_{0}^{j}$ é uma constante real não nula 
para $j=1,2$, quando $l \in\{1,2,3\}$. Aqui a função $h: \mathbb{R}^{2} \rightarrow \mathbb{R}$ é dada por

$$
h_{l}(x, y)=\prod_{k=0}^{l-1}\left(y-\tan \left(\alpha+\frac{k \pi}{l}\right) x\right)
$$

O conjunto $h_{l}^{-1}(0)$ é o produto de $l$ retas passando pela origem e divide o plano em $2 l$ setores de ângulo $\frac{\pi}{l}$ quando $\alpha \in\left(-\frac{\pi}{l}, \frac{\pi}{l}\right)$.

Tal estudo proporcionou o seguinte resultado

Teorema 0.0.1. Assuma que para $j=1,2$, os polinômios $f_{n_{1}}^{j}(x), g_{n_{2}}^{j}(x)$ e $h_{n_{3}}^{j}(x)$ têm grau $n_{1} \geq 1, n_{2} \geq 1$ e $n_{3} \geq 1$ respectivamente, $d_{0}^{j}$ é uma constante não nula e $l \in\{1,2,3\}$. Então para $|\varepsilon|$ suficientemente pequeno o número máximo de ciclos limites do sistema diferencial generalizado descontínuo do tipo Kukles (4.2.3) que bifurcam do centro linear $\dot{x}=y, \dot{y}=-x$, pela teoria do averaging, é $m(l)$, onde
i) $m(1)=\max \left\{2\left[\frac{n_{1}}{2}\right], n_{2}+1,2\left[\frac{n_{3}+2}{2}\right], 3\right\}$;
ii) $m(2)=\max \left\{\left[\frac{n_{1}-1}{2}\right],\left[\frac{n_{2}}{2}\right],\left[\frac{n_{3}+1}{2}\right], 1\right\}$;
iii) $m(3)=\max \left\{2\left[\frac{n_{1}}{2}\right], n_{2}+1,2\left[\frac{n_{3}+2}{2}\right], 3\right\}-1$. 


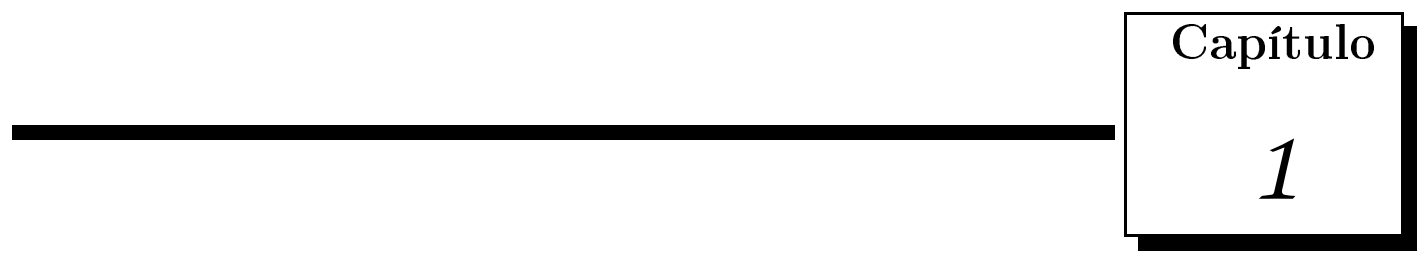

\section{Aspectos gerais}

Neste primeiro capítulo apresentamos definições, resultados e técnicas introdutórias e básicas da teoria qualitativa de equações diferenciais. O objetivo principal desse capítulo é fornecer ferramentas e motivação para o estudo realizado nos demais capítulos dessa dissertação.

Aqui está apresentado o teorema do averaging no caso suave. O resultado conta o número de soluções periódicas em sistemas do tipo $\dot{x}(t)=\varepsilon f^{1}(x, t)+\varepsilon^{2} f^{2}(x, t, \varepsilon)$ quando $f^{1}$ e $f^{2}$ são contínuas.

\subsection{Campos vetoriais e fluxos}

Definição 1.1.1. Um campo vetorial de classe $C^{k}, k=1,2, \ldots$ ou $k=\infty$, é uma aplicação $X: \Omega \rightarrow \mathbb{R}^{n}$ de classe $C^{k}$, onde $\Omega \subset \mathbb{R}^{n}$ é aberto.

Ao campo vetorial $X$ está associado a equação diferencial

$$
\dot{x}=X(x)
$$

onde ponto denota a derivada com respeito ao tempo $t$. 
As aplicações diferenciáveis $\varphi: I \rightarrow \Omega$ onde $I$ é um intervalo da reta, tais que

$$
\frac{d \varphi}{d t}(t)=X(\varphi(t)) \quad \text { para todo } t \in I
$$

são soluções da equação (1.1.1) chamadas trajetórias ou curvas integrais de $X$.

Se $x_{0}$ é um ponto tal que $X\left(x_{0}\right)=0$, então $x_{0}$ é dito ponto singular. Caso contrário é chamado ponto regular.

Uma curva integral $\varphi: I \rightarrow \Omega$ de $X$ é chamada maximal se para toda curva integral $\psi: J \rightarrow \Omega$ tal que $I \subseteq J$ e $\varphi=\psi \mid I$ então $I=J$ e consequentemente $\varphi=\psi$. Nesse caso o intervalo $I$ é também chamado de intervalo maximal.

Segue do Teorema de existência e unicidade de soluções que o sistema (1.1.1) sob a condição $x(0)=x_{0}$ admite unica solução maximal de classe $C^{k}$ passando por $x_{0}$. Denotamos por $I_{x_{0}}$ o intervalo maximal dessa solução.

Definição 1.1.2. Seja $\varphi: I_{x_{0}} \rightarrow \Omega$ uma solução maximal (regular ou constante). A imagem da curva $\gamma_{\varphi}=\left\{\varphi(t) ; t \in I_{x_{0}}\right\} \subset \Omega$ munida da orientação induzida por $\varphi$ é chamada de órbita associada à solução maximal $\varphi$.

De (1.1.2), $\varphi$ é uma curva integral de $X$ se e somente se seu vetor velocidade $\dot{\varphi}(t)$ em $t$ coincide com o valor do campo $X$ em $\varphi(t)$. A interpretação geométrica desse fato é dada na Figura 1.1.

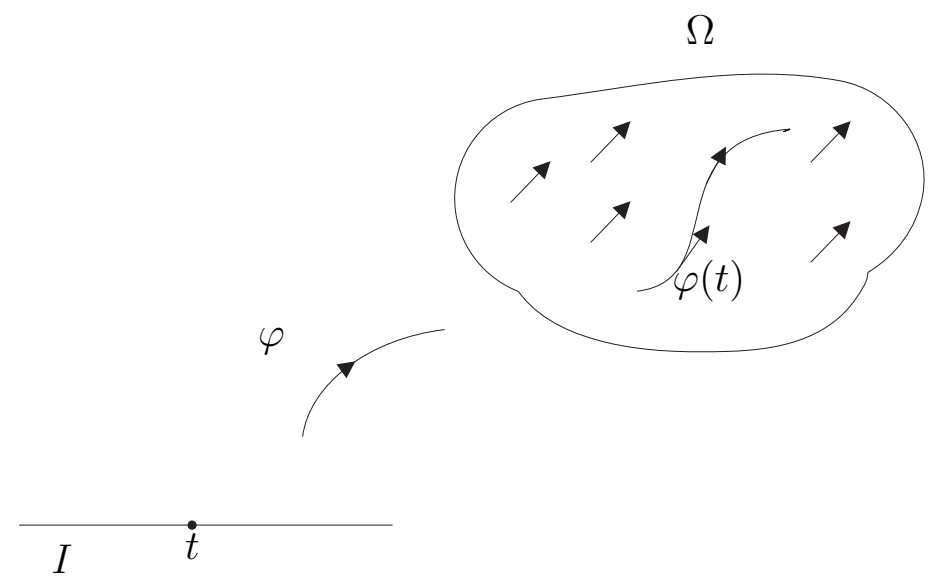

Figura 1.1: Relação entre campo de vetores e sua curva integral 
Definição 1.1.3. Considere o conjunto $D=\left\{(t, x): x \in \Omega, t \in I_{x}\right\}$ que é aberto em $\mathbb{R}^{n+1}$. A aplicação $\varphi: D \rightarrow \Omega$ dada por $\varphi(t, x)=\varphi_{x}(t)$ é chamada de fluxo gerado por $X$.

Proposição 1.1.4. [36] A aplicação $\varphi: D \rightarrow \Omega$ é de classe $C^{k}$.

Definição 1.1.5. Seja $\varphi_{x}(t)$ uma curva integral de $X$. Dizemos que ela é periódica se existe um número real $T>0$ tal que $\varphi_{x}(t+T)=\varphi_{x}(t)$, para todo $t \in \mathbb{R}$.

\subsection{Retrato de fase de um sistema diferencial}

Após algumas definições, apresentaremos uma discussão sobre o retrato de fase de um sistema diferencial planar. Denotaremos por $\gamma_{p}$ a órbita passando pelo ponto $p \in \mathbb{R}^{n}$.

Observação 1.2.1. A órbita passando por qualquer ponto $q \in \gamma_{p}$ coincide com $\gamma_{p}$, i.e., se $q \in \gamma_{p}$, então $\gamma_{q}=\gamma_{p}$.

Pela Observação 1.2.1 segue que se $q \in \gamma_{p}$, então existe $t_{1} \in I_{p}$ tal que

$$
q=\varphi\left(t_{1}, p\right), \quad \varphi(t, q)=\varphi\left(t+t_{1}, p\right) \quad \text { e } \quad I_{p}-t_{1}=I_{q}
$$

Em outras palavras, dadas duas órbitas de $X$ então elas coincidem ou são disjuntas.

O próximo resultado afirma que, dada uma solução $\varphi$ de um campo de vetores $X$, ela é ou um ponto, ou uma linha, ou uma órbita periódica.

Proposição 1.2.2. [6] Se $\varphi$ é uma solução maximal $C^{k}$ do sistema diferencial (1.1.1) com $n=2$, então uma das alternativas é válida.

(i) $\varphi$ é uma bijeção sobre sua imagem;

(ii) $I=\mathbb{R}, \varphi$ é uma função constante e $\gamma_{\varphi}$ é um ponto;

(iii) $I=\mathbb{R}$ e $\varphi$ é uma função periódica de período minimal $T$ (i.e. existe um real $T>0$ tal que $\varphi(t+T)=\varphi(t)$, para todo $t \in \mathbb{R}$, e $\varphi\left(t_{1}\right) \neq \varphi\left(t_{2}\right)$, se $\left.\left|t_{1}-t_{2}\right|<T\right)$.

Observação 1.2.3. Nas afirmações (i) e (iii) da Proposição 1.2.2 podemos adicionar que $\gamma_{\varphi}$ é $C^{k}$-difeomorfa a $\mathbb{R}$ e que $\gamma_{\varphi}$ é $C^{k}$-difeomorfa a $\mathbb{S}^{1}$, respectivamente. 
Definição 1.2.4. O retrato de fase de um campo de vetores $X: \Omega \rightarrow \mathbb{R}^{2}$ é o conjunto das órbitas orientadas de $X$. Ele consiste de singularidades e órbitas regulares, orientadas de acordo com as soluções maximais que as descrevem.

Um tipo especial de sistema diferencial é dado pelo modelo abaixo

$$
\left(\begin{array}{c}
\dot{q}_{i} \\
\dot{p}_{i}
\end{array}\right)=\left(\begin{array}{c}
-\frac{\partial H}{\partial p_{i}} \\
\frac{\partial H}{\partial q_{i}}
\end{array}\right),
$$

onde $\left(q_{1}, \ldots, q_{n}, p_{1}, \ldots, p_{n}\right)$ são as coordenadas em $\mathbb{R}^{2 n}$ e $H: \mathbb{R}^{2 n} \rightarrow \mathbb{R}$ é uma função diferenciável. Tais sistemas recebem o nome de sistemas Hamiltonianos com $n$ graus de liberdade. A função $H$ é chamada de função Hamiltoniana do sistema.

Exemplo 1.2.5. Seja $n=1$ e considere o sistema:

$$
\left\{\begin{array}{l}
\dot{x}=-x \\
\dot{y}=y
\end{array}\right.
$$

Esse é um sistema Hamiltoniano com função Hamiltoniana $H(x, y)=x y$ e grau de liberdade 1.

\subsection{Ciclos limites}

Definição 1.3.1. Dado um campo vetorial $X: \Omega \rightarrow \mathbb{R}^{2}$, onde $\Omega$ é um aberto de $\mathbb{R}^{2}$, entendemos por ciclo limite uma órbita periódica fechada de $X$ que seja isolada. Em outras palavras, uma órbita periódica $\gamma$ é um ciclo limite se existe uma vizinhança $V$ de tal forma que $\gamma$ seja a única órbita periódica contida em $V$.

Considere o seguinte campo:

$$
\left\{\begin{array}{l}
\dot{x}=P(x, y), \\
\dot{y}=Q(x, y),
\end{array}\right.
$$

onde $P, Q$ são polinômios nas variáveis $x$ e $y$. O grau de (1.3.1) é dado pelo máximo entre 
o grau de $P$ e o grau de $Q$. Dizemos que o sistema é não degenerado se m.d.c $(P, Q)=1$. A menos de menção contrária, trabalharemos com esse tipo de sistema.

\subsubsection{O XVI problema de Hilbert}

O XVI problema de Hilbert está entre os poucos problemas que permanecem em aberto da lista proposta por Hilbert em 1900. Ele trata dos ciclos limites em sistemas diferenciais polinomiais e pode ser dividido em duas partes. A segunda parte do problema tem o seguinte enunciado: Qual o número máximo de ciclos limites de um sistema do tipo (1.3.1)? E o que podemos dizer sobre as posições relativas dos ciclos limites?

O número máximo de ciclos limites de um sistema do tipo (1.3.1) é denotamos por $H(n)$, onde $n$ indica o grau do sistema.

Note que se $n=1$, então temos um sistema diferencial planar linear e este por sua vez não possui ciclos limites (de fato, quando temos um sistema diferencial planar linear, as únicas órbitas periódicas possíveis ocorrem quando temos uma singularidade do tipo centro e tais órbitas não são isoladas). Sendo assim este trabalho estará concentrado em sistemas diferenciais polinomiais de grau maior ou igual a 2 .

Écalle [13] e Il'Yashenko [19] mostraram que o número de ciclos limites $H(n)$ de um sistema diferencial polinomial planar é sempre finito, mas não existem resultados que mostrem uma cota uniforme para esse número. Em contrapartida, há um exemplo em [33] que mostra que se o campo não for polinomial, o número de ciclos limites pode ser infinito. Com relação à configuração dos ciclos limites em sistemas diferenciais polinomiais planares, há um resultado geral que pode ser encontrado em [29].

No que segue, daremos uma série de definições que serão úteis para avançarmos na discussão sobre ciclos limites.

Definição 1.3.2. Uma configuração de ciclos limites é um conjunto finito de curvas fechadas simples $C=\left\{\gamma_{1}, \ldots, \gamma_{m}\right\}$ tais que $\gamma_{i} \cap \gamma_{j}=\emptyset, \forall i, j$.

Definição 1.3.3. Uma curva fechada é chamada algébrica se for uma componente conexa do conjunto de zeros de alguma função polinomial. 
O conjunto $C=\left\{\gamma_{1}, \ldots, \gamma_{m}\right\}$ será chamado de configuração de ciclos limites algébricos se as curvas fechadas $\gamma_{i}$ forem ciclos limites algébricos.

Exemplo 1.3.4. Considere o sistema diferencial

$$
\left\{\begin{array}{l}
\dot{x}=y+x-x\left(x^{2}+y^{2}\right), \\
\dot{y}=-x+y-y\left(x^{2}+y^{2}\right),
\end{array}\right.
$$

O seu campo de vetores tem a seguinte forma

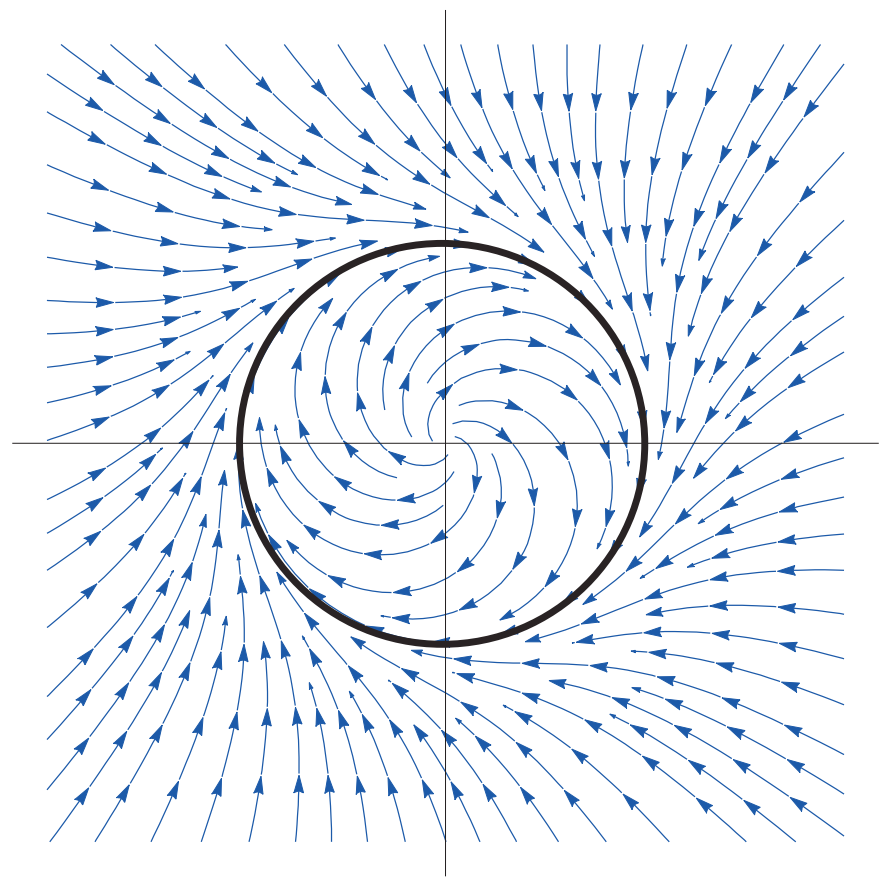

Figura 1.2: Campo vetorial do sistema (1.3.2).

O ciclo limite é formado pelo conjunto de zeros da função polinomial $f(x, y)=x^{2}+$ $y^{2}-1$.

Definição 1.3.5. Dada uma configuração de ciclos limites $C=\left\{\gamma_{1}, \ldots, \gamma_{m}\right\}$, a curva $\gamma_{i}$ será chamada primária se não existir nenhuma outra curva $\gamma_{j}$ de $C$ que esteja contida no interior(no sentido de Jordan) de $\gamma_{i}$.

Definição 1.3.6. Sejam $C=\left\{\gamma_{1}, \ldots, \gamma_{n}\right\}$ e $C^{\prime}=\left\{\gamma_{1}^{\prime}, \ldots, \gamma_{m}^{\prime}\right\}$ duas configurações de ciclos limites. Dizemos que $C$ e $C^{\prime}$ são topologicamente equivalentes, ou simplesmente equiva- 
lentes, se existir um homeomorfismo $h: C \rightarrow C^{\prime}$ tal que

$$
h\left(\bigcup_{i=1}^{n} \gamma_{i}\right)=\bigcup_{j=1}^{m} \gamma_{j}^{\prime}
$$

Como homeomorfismos preservam o número de componentes conexas, temos que se $C$ e $C^{\prime}$ são equivalentes, então $m=n$.

Dada uma configuração de cilos limites $C$, dizemos que um sistema realiza a configuração de ciclos limites $C$ se o conjunto de todos os seus ciclos limites for equivalente a $C$. Nesse sentido, temos um forte resultado:

Teorema 1.3.7. [6] Sejam $C=\left\{\gamma_{1}, \ldots \gamma_{m}\right\}$ uma configuração de ciclos limites e $r$ o número de ciclos limites primários de $C$. Então $C$ é realizável como ciclos limites algébricos por um sistema polinomial planar do tipo (1.3.1) de grau menor ou igual a $2(m+r)-1$.

Exemplo 1.3.8. Se considerarmos a configuração dada pela Figura 1.3, temos $m=6$ e $r=3$ e então pelo Teorema 1.3.7 existe um campo polinomial de grau menor ou igual a 17 cujo conjunto de ciclos limites é equivalente ao dado.
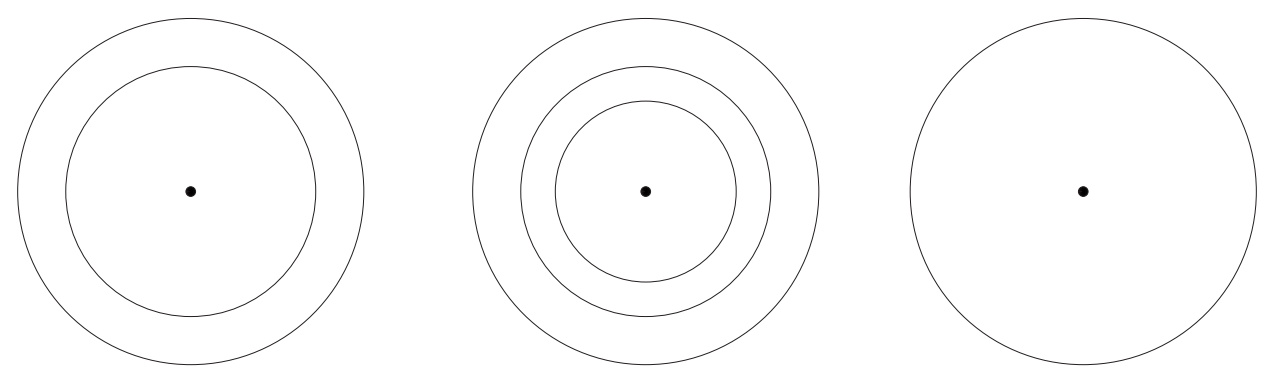

Figura 1.3: Configuração qualquer de ciclos limites

Podemos notar que nesse exemplo simples, a cota superior para o grau do sistema é muito alta. Já existem resultados no sentido de diminuir essa cota no entanto mais hipóteses são necessárias.

\subsubsection{A versão fraca do XVI problema de Hilbert}

É importante que ressaltemos a dificuldade do problema proposto por Hilbert. Apesar de ter sido investigado por centenas de pesquisadores, ele, juntamente com a hipótese de 
Riemann, são os únicos dois problemas abertos dos 23 propostos por Hilbert em 1900. Há uma versão mais fraca desse problema, proposta por Arnol'd [3, 5]: Qual o número máximo de ciclos limites que bifurcam de um centro?

A expressão "bifurca de um centro" significa que o ciclo limite é uma órbita periódica que se manteve estável após uma perturbação de um sistema que possuía uma singularidade do tipo centro. Daremos a definição formal mais a frente.

Um dos métodos usados para solucionar a versão fraca do XVI problema de Hilbert é o método das integrais abelianas. Este método será detalhado na Seção 1.4.

Definição 1.3.9. Seja $U$ um subconjunto aberto e denso de $\mathbb{R}^{2}$. Se uma função diferenciável não constante $H: U \rightarrow \mathbb{R}$ satisfaz $H(x(t), y(t))=$ cte, para todo $t$ tal que $(x(t), y(t))$ é solução de (1.3.1), então $H$ é chamada de integral primeira do sistema em $U$.

Proposição 1.3.10. Uma função $H: U \rightarrow \mathbb{R}$ é integral primeira de um sistema se, $e$ somente se,

$$
P(x, y) \frac{\partial H}{\partial x}(x, y)+Q(x, y) \frac{\partial H}{\partial y}(x, y)=0
$$

para todo $(x, y)$ em $U$.

Demonstração: Seja $(x(t), y(t))$ uma solução para (1.3.1). Por definição, $H$ é integral primeira se, e somente se,

$$
H(x(t), y(t))=c t e .
$$

Derivando $H$ em relação a $t$, obtemos:

$$
\dot{x} \frac{\partial H}{\partial x}+\dot{y} \frac{\partial H}{\partial y}=0
$$

e portanto,

$$
P(x, y) \frac{\partial H}{\partial x}(x, y)+Q(x, y) \frac{\partial H}{\partial y}(x, y)=0
$$

para todo $(x, y) \in U$ que é solução do sistema. Como o sistema está definido em todo o $\mathbb{R}^{2}$ e o Teorema da Existência e Unicidade nos garante que para cada ponto $(x, y)$ existe uma única solução do sistema, segue que a igualdade é válida para todo ponto de $U$. 
Definição 1.3.11. Um sistema planar é dito integrável se admite integral primeira $H$.

Observação 1.3.12. Note que se $H$ é integral primeira do sistema, então $\frac{1}{H}$ (nos pontos onde $H$ não se anula) e $H+C$, onde $C$ é uma constante real, também o são.

De fato, escrevendo $G=\frac{1}{H}$, temos $\frac{\partial G}{\partial x}=-\frac{\partial H}{\partial x} \frac{1}{H^{2}}$ e $\frac{\partial G}{\partial y}=-\frac{\partial H}{\partial y} \frac{1}{H^{2}}$. Logo,

$$
P(x, y) \frac{\partial G}{\partial x}(x, y)+Q(x, y) \frac{\partial G}{\partial y}(x, y)=\frac{1}{H^{2}}\left(-P(x, y) \frac{\partial H}{\partial x}(x, y)-Q(x, y) \frac{\partial H}{\partial y}(x, y)\right)=0
$$

Analogamente para $H+C$.

Definição 1.3.13. Dada $f \in \mathbb{R}[x, y]$, diremos que a curva de nivel $f(x, y)=0$ é uma curva algébrica invariante do sistema se existir uma função $K \in \mathbb{R}[x, y]$ tal que a seguinte igualdade se verifique

$$
P \frac{\partial f}{\partial x}+Q \frac{\partial f}{\partial y}=K f
$$

Observação 1.3.14. Se $f$ é curva algébrica invariante $e\left(x_{0}, y_{0}\right)$ é um ponto tal que $f\left(x_{0}, y_{0}\right)=0$ então $P \frac{\partial f}{\partial x}+Q \frac{\partial f}{\partial y}\left(x_{0}, y_{0}\right)=0$, isto é, se $X=(P, Q)$ temos $\langle X, \nabla f\rangle\left(x_{0}, y_{0}\right)=$ 0 e portanto, $X\left(x_{0}, y_{0}\right) \perp \nabla f\left(x_{0}, y_{0}\right)$. Logo, em cada ponto da curva o campo é paralelo ao vetor tangente de $f$ e se uma órbita intercepta a curva $f=0$, então ela está inteiramente contida na curva. Isso justifica o termo "invariante".

Uma integral primeira $H$ é chamada de integral primeira racional de grau $m$ se ela se escreve como quociente de dois polinômios coprimos cujo maior grau entre eles é $m$.

Observação 1.3.15. Se $H=\frac{H_{1}}{H_{2}}$ é integral primeira racional do sistema, então $H_{1}=0$ e $\mathrm{H}_{2}=0$ são curvas algébricas invariantes do mesmo sistema.

De fato, basta notar que, como $H$ é integral primeira do sistema,

$$
P \frac{\partial H}{\partial x}+Q \frac{\partial H}{\partial y}=0
$$

Por outro lado, $\frac{\partial H}{\partial x}=\left(\frac{\partial H_{1}}{\partial x} H_{2}-\frac{\partial H_{2}}{\partial x} H_{1}\right) \frac{1}{H_{2}^{2}} e \frac{\partial H}{\partial y}=\left(\frac{\partial H_{1}}{\partial y} H_{2}-\frac{\partial H_{2}}{\partial y} H_{1}\right) \frac{1}{H_{2}^{2}}$.

$\operatorname{Logo}$,

$$
P\left(\frac{\partial H_{1}}{\partial x} H_{2}-\frac{\partial H_{2}}{\partial x} H_{1}\right) \frac{1}{H_{2}^{2}}+Q\left(\frac{\partial H_{1}}{\partial y} H_{2}-\frac{\partial H_{2}}{\partial y} H_{1}\right) \frac{1}{H_{2}^{2}}=0 .
$$


e portanto,

$$
P \frac{\partial H_{1}}{\partial x}+Q \frac{\partial H_{1}}{\partial y}=\frac{1}{H_{2}}\left(Q \frac{\partial H_{2}}{\partial y}+P \frac{\partial H_{2}}{\partial x}\right) H_{1} .
$$

Tomando $K(x, y)=\frac{1}{H_{2}}\left(Q \frac{\partial H_{2}}{\partial y}+P \frac{\partial H_{2}}{\partial x}\right)$ concluímos que $H_{1}=0$ é uma curva algébrica invariante do sistema. O raciocínio é análogo para $\mathrm{H}_{2}$.

Note que a Observação 1.3.15 fornece uma maneira de construir integrais primeiras a partir de duas curvas invariantes.

\subsection{Integrais Abelianas}

A fim de descrever o método das integrais abelianas, vamos relembrar o conceito de 1-forma em $\mathbb{R}^{n}$.

Definição 1.4.1. [4] Uma 1-forma em $\mathbb{R}^{n}$ é uma aplicação linear $\omega: \mathbb{R}^{n} \rightarrow \mathbb{R}$. O espaço de todas as 1-formas diferenciais em $\mathbb{R}^{n}$ é um espaço vetorial de dimensão $n$, chamado dual de $\mathbb{R}^{n}$ e denotado por $\left(\mathbb{R}^{n}\right)^{*}$.

Queremos uma base conveniente para o dual de $\mathbb{R}^{n}$. Como é um espaço de funções, então obviamente a base será formada por funções lineares. Considere para cada $i \in$ $\{1, \ldots, n\}$ a 1 -forma

$$
d x_{i}: \mathbb{R}^{n} \rightarrow \mathbb{R}
$$

que a cada vetor $\xi=\left(\xi_{1}, \ldots, \xi_{n}\right) \in \mathbb{R}^{n}$ associa sua $i$-ésima coordenada, isto é, $d x_{i}(\xi)=\xi_{i}$. É fácil ver que o conjunto $\left\{d x_{1}, \ldots, d x_{n}\right\}$ é linearmente independente e portanto temos uma base para o espaço dual de $\mathbb{R}^{n}$. Sendo assim, qualquer 1-forma pode ser escrita como

$$
\omega(\xi)=\lambda_{1}(\xi) d x_{1}+\ldots+\lambda_{n}(\xi) d x_{n}
$$

onde $\lambda_{i}: \mathbb{R}^{n} \rightarrow \mathbb{R}$ são funções diferenciáveis.

Observação 1.4.2. Por 1-forma racional entendemos ser uma 1-forma cujos $\lambda_{i}^{\prime} s$ são funções racionais. 
Definição 1.4.3. Uma integral abeliana é integral de linha de uma 1-forma racional em $\mathbb{R}^{2}$ ao longo de uma órbita periódica algébrica.

No que segue, chamaremos as curvas fechadas simples de ovais.

Seja $H \in \mathbb{R}[x, y]$ um polinômio de grau maior ou igual a dois que possua uma família contínua de ovais consistindo de curvas de nível de $H$ :

$$
\gamma_{h}=\left\{(x, y) \in \mathbb{R}^{2}: H(x, y)=h\right\},
$$

para $h \in(a, b)$.

Dados $f$ e $g$ polinômios de grau maior ou igual a 2, defina a seguinte 1-forma polinomial

$$
\omega=f(x, y) d y-g(x, y) d x
$$

Há uma versão do XVI problema de Hilbert, proposta por Arnol'd que pode ser escrita como: Dados números naturais $m, n$, encontre o número máximo de zeros isolados da integral abeliana

$$
I(h)=\oint_{\gamma_{h}} \omega .
$$

Pergunta 1.4.4. Qual a relação entre esse problema e o de contar o número de ciclos limites do sistema $\dot{x}=f(x, y)$ e $\dot{y}=g(x, y)$ ?

Os próximos passos nos conduzirão à uma equivalência entre esses dois assuntos. Dado $H(x, y)$ um polinômio de grau $m$, a ele associamos o campo $X_{H}$ :

$$
\left\{\begin{array}{l}
\dot{x}=-\frac{\partial H}{\partial y}(x, y) \\
\dot{y}=\frac{\partial H}{\partial x}(x, y)
\end{array}\right.
$$

Perturbando o sistema (1.4.1) por polinômios $f$ e $g$ com $\varepsilon$ suficientemente pequeno, obtémse um novo sistema $X_{H, \varepsilon}$

$$
\left\{\begin{array}{l}
\dot{x}=-\frac{\partial H}{\partial y}(x, y)+\varepsilon f(x, y) \\
\dot{y}=\frac{\partial H}{\partial x}(x, y)+\varepsilon g(x, y) .
\end{array}\right.
$$


Suponhamos que exista uma família contínua de ovais $\left\{\gamma_{h}\right\} \subset H^{-1}(h)$ que dependa continuamente do parâmetro $h$ em um intervalo $(a, b)$, isto é, se $h_{1}$ está próximo de $h_{2}$, então $\gamma_{1}$ está próxima de $\gamma_{2}$ e defina a integral abeliana:

$$
I(h)=\oint_{\gamma_{h}} f(x, y) d y-g(x, y) d x
$$

Observação 1.4.5. Para cada $h \in(a, b)$ a curva $\gamma_{h}$ é uma órbita periódica do sistema Hamiltoniano (1.4.1).

De fato, se $\gamma_{h}(t)=\left(x_{h}(t), y_{h}(t)\right)$ então $H\left(x_{h}(t), y_{h}(t)\right)=h$. Derivando em relação à $t$ concluimos que $\left\langle\nabla H, \dot{\gamma}_{h}(t)\right\rangle=0$. Logo, a curva intercepta órbitas do campo (1.4.1) e estão inteiramente contidas na curva fechada.

Pergunta 1.4.6. Quantas ovais mantém-se órbitas periódicas do sistema perturbado (1.4.2) para $\varepsilon$ suficientemente pequeno?

Note que se conseguirmos responder à essa questão e a resposta for um número finito, teremos o número de ciclos limites do sistema (1.4.2).

Para responder a questão colocada vamos construir uma função chamada de função sucessão.

Dado o conjunto das curvas de nível $\left\{\gamma_{h}\right\}$, construímos um segmento $\sigma$ transversal a cada uma delas. Note que isso é possível pois como são curvas de nível, o vetor gradiente de $H$ é ortogonal ao vetor tangente $\dot{\gamma}_{h}(t)$, para cada $t$. Construa o segmento transversal $\sigma$ usando os vetores gradientes de $H$ e parametrize-o pela função $H$. Seja $\gamma(h, \varepsilon)$ o trecho da órbita do sistema perturbado (1.4.2) entre o ponto inicial $h$ em $\sigma$ e o próximo ponto de interseção $P(h, \varepsilon)$ com $\sigma$. O fato de existir o ponto $P(h, \varepsilon)$, decorre do fato de que se $\varepsilon$ é suficientemente pequeno, então a órbita $\gamma(h, \varepsilon)$ está próxima de $\gamma_{h}$ que é fechada. Tal processo é ilustrado na Figura 1.4

Definição 1.4.7. A diferença $d(h, \varepsilon)=P(h, \varepsilon)-h$ é chamada função sucessão.

Teorema 1.4.8. (Poincaré-Pontryagin) A função sucessão pode ser escrita como

$$
d(h, \varepsilon)=\varepsilon(I(h)+\varepsilon \Phi(h, \varepsilon))
$$




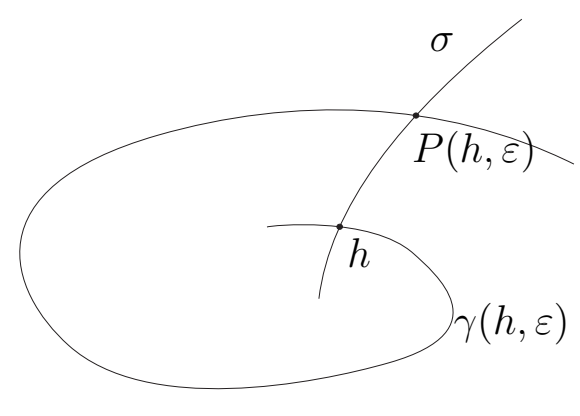

Figura 1.4: Função sucessão

quando $\varepsilon \rightarrow 0$, onde $\Phi(h, \varepsilon)$ é analítica e uniformemente limitada para $(h, \varepsilon)$ numa região compacta próxima a $(h, 0)$, com $h \in(a, b)$ e I(h) dada pela expressão (1.4.3).

Demonstração: Basta notar que, parametrizando $\sigma$ por $H$, a função de sucessão fica sendo a diferença, em relação à $H$, entre os pontos inicial e final de $\gamma(h, \varepsilon)$. Ou seja,

$$
d(h, \varepsilon)=\int_{\gamma(h, \varepsilon)} d H=\int_{\gamma(h, \varepsilon)}\left(\frac{\partial H}{\partial x} \frac{d x}{d t}+\frac{\partial H}{\partial y} \frac{d y}{d t}\right) d t .
$$

Mas, de (1.4.2), temos que $\dot{x}=-\frac{\partial H}{\partial y}+\varepsilon f$ e $\dot{y}=\frac{\partial H}{\partial x}+\varepsilon g$. Substituindo essas expressões obtemos

$$
\begin{gathered}
d(h, \varepsilon)=\int_{\gamma(h, \varepsilon)} \frac{\partial H}{\partial x}\left(-\frac{\partial H}{\partial y}+\varepsilon f\right)+\frac{\partial H}{\partial y}\left(\frac{\partial H}{\partial x}+\varepsilon g\right) d t \\
=\int_{\gamma(h, \varepsilon)}\left(\frac{\partial H}{\partial x} \varepsilon f+\frac{\partial H}{\partial y} \varepsilon g\right) d t \\
=\varepsilon \int_{\gamma(h, \varepsilon)}\left(\frac{\partial H}{\partial x} f+\frac{\partial H}{\partial y} g\right) d t .
\end{gathered}
$$

Por outro lado, de (1.4.1), temos que $\frac{\partial H}{\partial x}=\frac{d y}{d t}$ e $\frac{\partial H}{\partial y}=-\frac{d x}{d t}$. Logo,

$$
d(h, \varepsilon)=\varepsilon \int_{\gamma(h, \varepsilon)} f d y-g d x
$$

Mas $\gamma(h, \varepsilon)$ converge uniformemente para o compacto $\gamma_{h}$, e então $d(h, \varepsilon)=\varepsilon(I(h)+o(\varepsilon))$, como queríamos.

Observação 1.4.9. Como estamos parametrizando $\sigma$ pela função $H$, o número de zeros 
da integral independe da seção transversal escolhida.

Definição 1.4.10. [36] Um ciclo limite $\Gamma$ diz-se um atrator periódico (ou orbitalmente estável) quando todas as demais órbitas numa vizinhança $V_{\Gamma}$ tenderem a $\Gamma$.

O próximo teorema, demonstrado em [36], dá condições para uma órbita periódica ser instável ou estável.

Teorema 1.4.11. Sejam $\Omega \subset \mathbb{R}^{2}$ um aberto e $X=(P, Q): \Omega \rightarrow \mathbb{R}^{2}$ um campo vetorial. Sejam $\Gamma$ uma órbita periódica de $X$ de período $T$ e d a função sucessão. Então

$$
d^{\prime}(h, \varepsilon)=\exp \left(\int_{0}^{T} \operatorname{div} X(\Gamma(t)) d t\right)
$$

onde $\operatorname{div} X(x, y)=\left(\frac{\partial P}{\partial x}+\frac{\partial Q}{\partial y}\right)(x, y)$. Em particular, se $\int_{0}^{T} \operatorname{div} X(\Gamma(t)) d t<0$ então $\Gamma$ é estável e se $\int_{0}^{T} \operatorname{div} X(\Gamma(t)) d t>0, \Gamma$ é instável.

Definição 1.4.12. Um ciclo limite $\Gamma$ de período $T$ é dito hiperbólico se

$$
\int_{0}^{T} \operatorname{div} X(\Gamma(t)) d t \neq 0 .
$$

Definição 1.4.13. (i) Se existirem $h^{*} \in(a, b) e \varepsilon^{*}>0$ tais que $X_{H, \varepsilon}$ tenha um ciclo limite $\Gamma_{\varepsilon}$ para $0<|\varepsilon|<\varepsilon^{*}$ e $\Gamma_{\varepsilon}$ tende a $\Gamma_{h^{*}}$ quando $\varepsilon \rightarrow 0$, então diremos que $\Gamma_{\varepsilon}$ bifurca-se de $\Gamma_{h^{*}}$.

(ii) Diremos que um ciclo limite $\Gamma$ de $X_{H, \varepsilon}$ bifurca-se do anel $\bigcup_{h \in(a, b)} \gamma_{h}$ de $X_{H}$, se existir $h \in(a, b)$ tal que $\Gamma$ bifurca-se de $\gamma_{h}$.

Finalmente, podemos enunciar o teorema que relaciona o número de zeros da integral abeliana com o número de ciclos limites do sistema perturbado. Dado que o objetivo dessa seção é fazermos uma introdução ao método das integrais abelianas omitiremos sua demonstração, que pode ser encontrada com detalhes em [34].

Teorema 1.4.14. Suponhamos que $I(h)$ não seja identicamente nula para $h \in(a, b)$. Então, valem as seguintes afirmações: 
1) Se $X_{H, \varepsilon}$ possui um ciclo limite bifurcando-se de $\Gamma_{h^{*}}$ então $I\left(h^{*}\right)=0$.

2) Se existir $h^{*} \in(a, b)$ tal que $I\left(h^{*}\right)=0$ e $I^{\prime}\left(h^{*}\right) \neq 0$, então $X_{H, \varepsilon}$ tem um único ciclo limite bifurcando-se de $\Gamma_{h^{*}}$. Além disso, o ciclo é hiperbólico.

3) Se existir $h^{*} \in(a, b)$ tal que $I\left(h^{*}\right)=I^{\prime}\left(h^{*}\right)=\ldots=I^{(k-1)}\left(h^{*}\right)=0$ e $I^{(k)}\left(h^{*}\right) \neq 0$, então $X_{H, \varepsilon}$ tem no máximo $k$ ciclos limites bifurcando-se da mesma $\gamma_{h^{*}}$, levando em consideração a multiplicidade dos ciclos limites.

4) O número total de ciclos limites de $X_{H, \varepsilon}$ (contando as multiplicidades), bifurcando-se do anel $\bigcup_{h \in(a, b)} \gamma_{h}$ de $X_{H}$, é limitado pelo número máximo de zeros isolados de $I(h)$, para $h \in(a, b)$ (levando em consideração as multiplicidades).

Exemplo 1.4.15. Considere o seguinte sistema planar

$$
\left\{\begin{array}{l}
\dot{x}=y \\
\dot{y}=-x+\varepsilon\left(1-x^{2}\right) y .
\end{array}\right.
$$

O sistema acima é uma perturbação de $X_{H}$ dado por

$$
\left\{\begin{array}{l}
\dot{x}=y \\
\dot{y}=-x
\end{array}\right.
$$

que é Hamiltoniano e cuja familía contínua de ovais é dada por

$$
\gamma_{h}=\left\{(x, y) \in \mathbb{R}^{2}: H(x, y)=x^{2}+y^{2}=h, h>0\right\}
$$

Do sistema perturbado $X_{H, \varepsilon}$ e usando a notação de 1-forma e integral abeliana temos $f(x, y)=0$ e $g(x, y)=y\left(1-x^{2}\right)$, logo a integral abeliana a ser resolvida é

$$
I(h)=\oint_{\gamma_{h}} y\left(1-x^{2}\right) d x
$$


Parametrizando a curva por $x=h \cos \theta$ e $y=h \operatorname{sen} \theta$ no intervalo $[0,2 \pi]$, segue que

$$
\begin{aligned}
I(h) & =\oint_{\gamma_{h}}\left(1-x^{2}\right) y d x=\int_{0}^{2 \pi}\left(1-h^{2} \cos ^{2} \theta\right)(h \operatorname{sen} \theta)(-h \operatorname{sen} \theta d \theta) \\
& =-\int_{0}^{2 \pi}\left(1-h^{2} \cos ^{2} \theta\right)\left(h^{2} \operatorname{sen}^{2} \theta\right) d \theta \\
& =-\int_{0}^{2 \pi}\left(h^{2} \operatorname{sen}^{2} \theta-h^{4} \operatorname{sen}^{2} \theta \cos ^{2} \theta\right) d \theta \\
& =-\int_{0}^{2 \pi} h^{2} \operatorname{sen}^{2} \theta d \theta+\int_{0}^{2 \pi} h^{4}(\operatorname{sen} \theta \cos \theta)^{2} d \theta \\
& =\pi h^{2}\left(\frac{h^{2}}{4}-1\right)
\end{aligned}
$$

Logo, $I(h)=0 \Leftrightarrow h=0$ ou $h= \pm 2$. Como $h^{*}=0$ corresponde à singularidade do sistema, $h^{*}=2$ é o único zero positivo de $I(h)$.

Note ainda que $I^{\prime}(h)=2 \pi h\left(\frac{h^{2}}{2}-1\right)$ e portanto $I^{\prime}(2)=4 \pi \neq 0$. Assim, pelo teorema (1.4.14), segue que para $\varepsilon$ suficientemente pequeno, o sistema $X_{H, \varepsilon}$ possui um único ciclo limite hiperbólico que tende ao círculo de raio $\sqrt{2}$ quando $\varepsilon \rightarrow 0$.

Observação 1.4.16. O Teorema 1.4.14 nos dá o número de ciclos limites e suas posições relativas. Entretanto, para calcularmos o número exato de ciclos limites, é preciso resolver a integral e isso usualmente não é fácil, assim outras técnicas foram desenvolvidas. Nesse trabalho veremos uma segunda técnica, bastante empregada atualmente e conhecida como teoria do averaging.

\section{$1.5 \mathrm{O}$ método do averaging para sistemas diferenciais}

\section{contínuos}

\subsubsection{Averaging analítico}

Inicialmente o método do averaging foi desenvolvido com o objetivo de aproximar a solução de um sistema não autônomo pela solução de um sistema autônomo. Em [31] há 
uma descrição minuciosa do método, que é aplicado a sistemas do tipo

$$
\left\{\begin{array}{l}
\dot{x}=\varepsilon f^{1}(x, t)+\varepsilon^{2} f^{2}(x, t, \varepsilon) \\
x(0)=x_{0}
\end{array}\right.
$$

com $f^{1}$ e $f^{2}$ funções contínuas e $T$-periódicas.

No que segue, faremos uma introdução da técnica. Dado um sistema como em (1.5.1), considere o sistema promediado

$$
\left\{\begin{array}{l}
\dot{z}=\varepsilon f(z) \\
z(0)=x_{0}
\end{array}\right.
$$

onde $f(z)=\int_{0}^{T} f^{1}(z, s) d s$ é a média de $f^{1}(z, t)$ sobre seu período $T$ em $t$.

Observe que esse novo sistema é autônomo e foi obtido do primeiro. Sendo autônomo, temos as técnicas para resolvê-lo. O teorema principal desta seção nos garantirá que, sob certas condições, a solução de (1.5.2) aproxima bem a solução de (1.5.1).

Teorema 1.5.1. (Averaging de primeira ordem analítico) Suponhamos que $f^{1}$ seja Lipschitz, $f^{2}$ seja contínua e $\varepsilon, D$ e $L>0$ sejam tais que $D \subset \mathbb{R}^{n}$ é um subconjunto aberto, conexo e relativamente compacto que contenha $x_{0}$ de tal forma que se $0 \leq \varepsilon \leq \varepsilon_{0}$ então as soluções $x(t, \varepsilon)$ de (1.5.1) e $z(t, \varepsilon)$ de (1.5.2) permaneçam em $D$ para $0 \leq t \leq \frac{L}{\varepsilon}$. Sob essas condições, existe uma constante $c>0$ tal que

$$
\|x(t, \varepsilon)-z(t, \varepsilon)\|<c \varepsilon
$$

para $0 \leq \varepsilon \leq \varepsilon_{0}$ e $0 \leq t \leq \frac{L}{\varepsilon}$, onde $x(t, \varepsilon)$ é solução de (1.5.1) e z(t,e) é solução de $(1.5 .2)$.

A demonstração do Teorema 1.5.1, juntamente com algumas aplicações, pode ser encontrada por exemplo em [31] e [34].

Observação 1.5.2. A forma como o sistema (1.5.1) é apresentada chama-se forma padrão. Recentemente, Llibre, Novaes e Teixeira mostraram em [27] que a técnica do averaging pode ser aplicada a sistemas que não estejam na forma padrão, mas nesse caso a 
expressão da função promediada é diferente. Eles usaram o grau de Brouwer na demonstração do resultado, mesma ferramenta que usaremos na próxima seção.

\subsubsection{Averaging via grau de Brouwer}

Na seção anterior, foi apresentado um resumo sobre a técnica do averaging com uma abordagem analítica. A ideia agora é estudar a mesma técnica, só que de um ponto de vista topológico e utilizá-la para encontrar órbitas periódicas de sistemas diferenciais. A ferramenta usada será o grau de Brouwer e para isso precisaremos defini-lo e ver algumas de suas propriedades. Vale ressaltar que o objetivo do nosso trabalho é entender a técnica para sistemas diferenciais contínuos e depois estudá-la com maiores detalhes para sistemas de Filippov. Sendo assim, detalhes minuciosos sobre a teoria do grau de Brouwer serão omitidos, podendo ser encontrados em [1].

Antes de começarmos a teoria, fixemos algumas notações:

$-D_{x} f$ denota a matriz Jacobiana de $f$ com relação à variável $x$.

- $J_{f}(a)$ denota o determinante da Jacobiana da aplicação $f$ avaliada em $a$.

Considere um conjunto $\Omega \subset \mathbb{R}^{n}$ aberto e limitado e denote por $\bar{\Omega}$ o seu fecho. Sejam $f \in C^{1}\left(\bar{\Omega}, \mathbb{R}^{n}\right), S=\left\{x \in \bar{\Omega} ; J_{f}(x)=0\right\}$ e $y_{0} \in \mathbb{R}^{n}$ tal que $y_{0} \notin f(\partial \Omega) \cup f(S)$. Então, o grau topológico de Brouwer da função $f$ com relação a $\Omega$ no ponto $y_{0}$ é dado pelo número inteiro

$$
d_{B}\left(f, \Omega, y_{0}\right)=\sum_{x_{i} \in f^{-1}\left(\left\{y_{0}\right\}\right)} \operatorname{sign}\left(J_{f}\left(x_{i}\right)\right)
$$

onde

$$
\operatorname{sign}(x)= \begin{cases}1 & \text { se } x>0 \\ -1 & \text { se } x<0\end{cases}
$$

Observação 1.5.3. A definição do grau topológico de Brouwer também vale para funções de duas variáveis $f(z, \varepsilon)$. Nesse caso o grau de Brouwer é calculado para $\varepsilon$ fixado.

Observação 1.5.4. Dizer que uma função $f: \bar{\Omega} \rightarrow \mathbb{R}^{n}$ é de classe $C^{1}$ em $\bar{\Omega}$ significa dizer que para cada $x \in \partial \Omega$ existe uma função $F: U \rightarrow \mathbb{R}^{n}$ tal que $U \subset \mathbb{R}^{n}$ é aberto com $U \cap \bar{\Omega} \neq \emptyset$, tal que $F$ é diferenciável em $U$ e $F(U \cap \bar{\Omega})=f(U \cap \bar{\Omega})$. Definimos então $f^{\prime}(x)=F^{\prime}(x)$. 
Afirmação 1.5.5. $d_{B}: C^{1}\left(\bar{\Omega}, \mathbb{R}^{n}\right) \times \mathbb{R}^{n} \times B \rightarrow \mathbb{Z}$, onde $B=\mathbb{R}^{n}-\{f(\partial \Omega) \cup f(S)\}$ está bem definida, isto é, $d_{B}\left(f, \Omega, y_{0}\right)$ é finito.

Seja $x \in f^{-1}\left(\left\{y_{0}\right\}\right)$, por construção $J_{f}(x) \neq 0$, logo pelo Teorema da Aplicação Inversa, existem abertos $U_{x}$ e $V_{x}=f\left(U_{x}\right)$ tais que $x \in U_{x}, y_{0} \in V_{x}$ e $f_{\mid U_{x}}: U_{x} \rightarrow V_{x}$ é um difeomorfismo. Para cada $x \in f^{-1}\left(\left\{y_{0}\right\}\right)$ considere $B_{r_{x}}(x)$ contida em $U_{x}$ a bola aberta de centro $x$ e raio $r_{x}$. Assim,

$$
f^{-1}\left(\left\{y_{0}\right\}\right) \subset \bigcup_{x \in f^{-1}\left(\left\{y_{0}\right\}\right)} B_{r_{x}}(x)
$$

Como $f^{-1}\left(\left\{y_{0}\right\}\right)$ é fechado ( $f$ é contínua) e está contido em $\bar{\Omega}$ que é limitado segue que $f^{-1}\left(\left\{y_{0}\right\}\right)$ é compacto. Logo, pode ser coberto com um número finito de bolas abertas da cobertura anterior, isto é,

$$
f^{-1}\left(\left\{y_{0}\right\}\right) \subset \bigcup_{i=1}^{n} B_{r_{x_{i}}}\left(x_{i}\right) .
$$

Como havíamos tomado uma bola para cada $x \in f^{-1}\left(\left\{y_{0}\right\}\right)$, segue que, $f^{-1}\left(\left\{y_{0}\right\}\right)=$ $\left\{x_{1}, \ldots, x_{k}\right\}$ com $J_{f}\left(x_{j}\right) \neq 0$ para todo $j \in\left\{x_{1}, \ldots, x_{k}\right\}$.

Lema 1.5.6. $\operatorname{Se} d_{B}\left(f, \Omega, y_{0}\right) \neq 0$, então existe $x_{0} \in \Omega$ tal que $f\left(x_{0}\right)=y_{0}$.

Demonstração: $\operatorname{Se} d_{B}\left(f, \Omega, y_{0}\right) \neq 0$, então o somatório contém pelo menos uma parcela, isto é, não é vazio. Sendo assim, existe pelo menos um $x_{0} \in \Omega$ tal que $f\left(x_{0}\right)=y_{0}$.

Proposição 1.5.7. Valem as seguintes propriedades:

i) (Excisão) Seja $K \subset \bar{\Omega}$ um compacto e $y_{0} \notin f(K) \cup f(\partial \Omega)$. Então

$$
d_{B}\left(f, \Omega, y_{0}\right)=d_{B}\left(f, \Omega \backslash K, y_{0}\right) .
$$

ii) (Invariância por homotopia) Sejam $H \in C\left(\bar{\Omega} \times[0,1], \mathbb{R}^{n}\right)$ e $y_{0} \notin H(\partial \Omega \times[0,1])$. Então:

$$
d_{B}\left(H(\cdot, t), \Omega, y_{0}\right) \equiv c t e, \forall t \in[0,1]
$$

Afim de exemplificarmos a definição vista, calculemos o grau de Brouwer de algumas funções. 
Exemplo 1.5.8. Seja $\Omega=(-1,1) \subset \mathbb{R}, f: \bar{\Omega} \rightarrow \mathbb{R}$ definida por $f(x)=x^{2}$ e $y_{0} \in \mathbb{R}$.

Se $y_{0}<0$, então $f^{-1}\left(\left\{y_{0}\right\}\right)=\emptyset$ e portanto $d_{B}\left(f, \Omega, y_{0}\right)=0$.

Se $y_{0}>0$, então $f^{-1}\left(\left\{y_{0}\right\}\right)=\left\{-\sqrt{y_{0}}, \sqrt{y_{0}}\right\}$. Vemos que $f^{\prime}\left(-\sqrt{y_{0}}\right)=-2 \sqrt{y_{0}}<0$ e $f^{\prime}\left(\sqrt{y_{0}}\right)=2 \sqrt{y_{0}}>0$ o que implica que $f^{\prime}\left(-\sqrt{y_{0}}\right)$ inverte orientação e $f^{\prime}\left(\sqrt{y_{0}}\right)$ preserva orientação. Sendo assim,

$$
d_{B}\left(f, \Omega, y_{0}\right)=1-1=0 .
$$

Exemplo 1.5.9. Considere $\Omega=\left(0, \frac{5 \pi}{2}\right)$ e $f(x)=\operatorname{sen} x$.

Note que $\partial \Omega=\left\{0, \frac{5 \pi}{2}\right\}, f(\partial \Omega)=\{0,1\}$, assim, se $y_{0}=\frac{\sqrt{2}}{2}, y_{0} \notin f(\partial \Omega) \cup f(S)$ e portanto $d_{B}\left(f, \Omega, \frac{\sqrt{2}}{2}\right)$ está bem definido. Além disso $\operatorname{sen} x=\frac{\sqrt{2}}{2}$ em $\Omega$, se e só se $x=\frac{\pi}{4}, \frac{3 \pi}{4}, \frac{9 \pi}{4} e$ $f^{\prime}\left(\frac{\pi}{4}\right)=\frac{\sqrt{2}}{2}>0, f^{\prime}\left(\frac{3 \pi}{4}\right)=-\frac{\sqrt{2}}{2}<0$ e $f^{\prime}\left(\frac{9 \pi}{4}\right)=\frac{\sqrt{2}}{2}>0$. Logo,

$$
d_{B}\left(f, \Omega, \frac{\sqrt{2}}{2}\right)=1-1+1=1
$$

Lema 1.5.10. Consideremos as funções contínuas $f^{i}: \bar{\Omega} \rightarrow \mathbb{R}^{n}$, para $i=0,1, \ldots, k$, e $f, g, r: \bar{\Omega} \times\left[-\varepsilon_{0}, \varepsilon_{0}\right] \rightarrow \mathbb{R}^{n}$ dadas por

$$
\begin{gathered}
g(\cdot, \varepsilon)=f^{0}(\cdot)+\varepsilon f^{1}(\cdot)+\varepsilon^{2} f^{2}(\cdot)+\cdots+\varepsilon^{k} f^{k}(\cdot), \\
f(\cdot, \varepsilon)=g(\cdot, \varepsilon)+\varepsilon^{k+1} r(\cdot, \varepsilon) .
\end{gathered}
$$

Assumamos que $g(z, \varepsilon) \neq 0$ para todo $z \in \partial \Omega$ e $\varepsilon \in\left[-\varepsilon_{0}, \varepsilon_{0}\right] \backslash\{0\}$. Então, para $|\varepsilon|>0$ suficientemente pequeno, $d_{B}(f(\cdot, \varepsilon), \Omega, 0)$ está bem definido e

$$
d_{B}(f(\cdot, \varepsilon), \Omega, 0)=d_{B}(g(\cdot, \varepsilon), \Omega, 0) .
$$

Demonstração: A ideia é usar a propriedade da invariância por homotopia. Para cada $\varepsilon \in\left[-\varepsilon_{0}, \varepsilon_{0}\right] \backslash\{0\}$, considere a seguinte homotopia contínua para $0 \leq t \leq 1$,

$$
h_{t}(\cdot, \varepsilon)=g(\cdot, \varepsilon)+t(f(\cdot, \varepsilon)-g(\cdot, \varepsilon))
$$

Pela Proposição 1.5.7, item $i i$ ), precisamos provar que para $\varepsilon$ suficientemente pequeno, $0 \notin$ $h_{t}(\partial \Omega, \varepsilon)$ para todo $t \in[0,1]$. Vamos mostrar por absurdo. Observe primeiramente que 
não existe $x_{0} \in \partial \Omega$ tal que $h_{0}\left(x_{0}\right)=0$, pois isso implicaria que $g\left(x_{0}, \varepsilon\right)=0$, contradizendo a hipótese. Suponha então que exista $t_{0} \in(0,1]$ e $x_{0} \in \partial \Omega$ tal que $h_{t_{0}}\left(x_{0}, \varepsilon\right)=0$. Dado que $r$ é uma função contínua e $\bar{\Omega}$ é compacto, segue que existe $M>0$ tal que $|r(z, \varepsilon)| \leq M$, $z \in \bar{\Omega}$ e $\varepsilon \in\left(0, \varepsilon_{0}\right)$. Assim, se $h_{t_{0}}\left(x_{0}, \varepsilon\right)=0$,

$$
g\left(x_{0}, \varepsilon\right)+t_{0}\left(f\left(x_{0}, \varepsilon\right)-g\left(x_{0}, \varepsilon\right)\right)=0
$$

Então

$$
g\left(x_{0}, \varepsilon\right)+t_{0}\left(\varepsilon^{k+1} r\left(x_{0}, \varepsilon\right)\right)=0 \Rightarrow g\left(x_{0}, \varepsilon\right)=-t_{0}\left(\varepsilon^{k+1} r\left(x_{0}, \varepsilon\right)\right) .
$$

Portanto

$$
\left|g\left(x_{0}, \varepsilon\right)\right| \leq \varepsilon^{k+1} M
$$

Mas então, se $|\varepsilon|>0$ é suficientemente pequeno, temos $g\left(x_{0}, \varepsilon\right)=0$, contrariando a hipótese.

Observação 1.5.11. Se $g: D \rightarrow \mathbb{R}^{n}$ é uma função $C^{1}$, onde $D$ é um aberto de $\mathbb{R}^{n}$, com $g(a)=0$ e $J_{g}(a) \neq 0$ segue do Teorema da Aplicação Inversa que existe uma vizinhança $\Omega$ de a tal que $g(z) \neq 0$, para todo $z \in \bar{\Omega} \backslash\{a\}$. Nesse caso, segue então que $d_{B}(g, \Omega, 0) \in$ $\{-1,1\}$. Disto, concluimos que para verificar que $d_{B}(g, \Omega, 0) \neq 0$, basta olhar se $J_{g}(a)$ se anula ou não.

Exemplo 1.5.12. (Aplicação do Lema 1.5.10) O grau de Brouwer da função $f(x)=x^{2}$ é zero em qualquer vizinhança da origem.

De fato, seja $\varepsilon>0$ qualquer, $\Omega=(-2 \varepsilon, 2 \varepsilon)$ e $g(x)=x^{2}-\varepsilon^{2}$. Note que $g$ tem dois zeros em $\Omega$ : $-\varepsilon$ e $\varepsilon$. Temos $g^{\prime}(-\varepsilon)<0$ e $g^{\prime}(\varepsilon)>0$ e portanto $d_{B}(g, \Omega, 0)=0$. Pelo Lema 1.5.10, segue que $d_{B}(f, \Omega, 0)=0$.

Corolário 1.5.13. Suponhamos que as hipóteses do Lema 1.5.10 estejam satisfeitas para $k=0$ e que além disso, para $a \in D$ aberto com $f^{0}(a)=0$, exista uma vizinhança $\Omega$ de a tal que $f^{0}(z) \neq 0$, para todo $z \in \bar{\Omega} \backslash\{a\}$, e $d_{B}\left(f^{0}, \Omega, 0\right) \neq 0$. Então, pelo menos um ramo de zeros de f bifurca-se de a.

Demonstração: Se $f(a)=0$ então a função já tem um zero. Caso contrário, pelo Lema 1.5.10 segue que $d_{B}(f(\cdot, \varepsilon), \Omega, 0)=d_{B}\left(f^{0}(\cdot), \Omega, 0\right)$ e aplicando a propriedade da excisão, 
temos

$$
d_{B}(f(\cdot, \varepsilon), \Omega \backslash\{a\}, 0)=d_{B}(f(\cdot, \varepsilon), \Omega, 0)=d_{B}\left(f^{0}, \Omega, 0\right) \neq 0 .
$$

Assim, para cada vizinhança $G_{\mu}$ de $a$, temos $d_{B}\left(f, G_{\mu} \backslash\{a\}, 0\right)=d_{B}\left(f^{0}, G_{\mu}, 0\right) \neq 0$.

Logo, $f$ possui um zero(diferente de $a$ ), para cada vizinhança $G_{\mu} \subset G$ de $a$. Escolhemos $G_{\mu}$ de tal forma que $G_{\mu} \rightarrow\{a\}$ quando $\mu \rightarrow 0$. Portanto, para $\varepsilon$ suficientemente pequeno, a função $f(\cdot, \varepsilon)$ possui pelo menos um zero $a_{\varepsilon} \in G_{\mu}$, o qual podemos escolher de tal forma que $a_{\varepsilon} \rightarrow a$ quando $\varepsilon \rightarrow 0$.

Como já foi observado, o averaging é uma técnica que permite que encontremos órbitas periódicas de sistemas através dos zeros de uma determinada função. Buica e Llibre [11] buscaram, através do Lema 1.5.10, respostas ao problema de encontrar zeros de uma função conveniente $f: D \times\left(-\varepsilon_{f}, \varepsilon_{f}\right) \rightarrow \mathbb{R}^{n}$. O procedimento é descrito abaixo.

Seja $D$ um subconjunto aberto de $\mathbb{R}^{n}$ e $f, g$ duas funções que satisfaçam as hipóteses do Lema 1.5.10. Primeiro, encontre os zeros de $f^{0}$. Dado $a \in D$ tal que $f^{0}(a)=0$, se existir uma vizinhança $\Omega$ de $a$ tal que $d_{B}\left(f^{0}, \Omega, 0\right) \neq 0$, então, para $|\varepsilon|>0$ suficientemente pequeno, $f(\cdot, \varepsilon)$ possuirá pelo menos um zero em $\Omega$.

Entretanto, se o grau de Brouwer de $f^{0}$ for zero em pequenas vizinhanças de $a$ ou se não puder ser calculado (o que inclui a possibilidade de $f^{0}$ ser identicamente nula), prosseguimos com o estudo de $f^{0}+\varepsilon f^{1}$ em alguma vizinhança pequena de $a$ e para $\varepsilon$ suficientemente pequeno.

Suponha que exista $a_{1 \varepsilon}$ que seja zero de $f^{0}+\varepsilon f^{1}$ e um subconjunto aberto e limitado $\Omega$ tais que $a_{1 \varepsilon} \in \Omega$ para cada $\varepsilon \neq 0$ suficientemente pequeno e $d_{B}\left(f^{0}+\varepsilon f^{1}, G, 0\right) \neq 0$.

Assim, pelo Lema 1.5.10, $f(\cdot, \varepsilon)$ possui pelo menos um zero em $\Omega$. Note que há a possibilidade de existir outros zeros de $f^{0}+\varepsilon f^{1}$ na mesma vizinhança de $a$, além de $a_{1 \varepsilon}$.

Se $d_{B}\left(f^{0}+\varepsilon f^{1}, G, 0\right)=0$, prosseguimos o estudo analogamente para a função $f^{0}+$ $\varepsilon f^{1}+\varepsilon^{2} f^{2}$, e assim por diante. Esse processo mostra qual é a ordem do averaging que deve ser aplicada. O próximo exemplo ilustrará esse raciocínio.

Exemplo 1.5.14. Seja $f: \mathbb{R}^{2} \rightarrow \mathbb{R}$ dada por:

$$
f(z, \varepsilon)=z^{2}-\varepsilon^{2}+\varepsilon^{3} r(z, \varepsilon)
$$


Vamos estimar o seu número de zeros. Segundo as hipóteses do Lema 1.5.10, temos $f^{0}(z)=z^{2}, f^{1}(z)=0, f^{2}(z)=-1$ e o grau de $f^{0}$ é zero para qualquer vizinhança da origem. Aplicando o processo acima, devemos estudar os zeros de

$$
\left(f^{0}+\varepsilon f^{1}+\varepsilon^{2} f^{2}\right)(z)=z^{2}-\varepsilon^{2} .
$$

Essa função por sua vez tem dois zeros e seu grau é não nulo: De fato, seja $\varepsilon_{0}>0$ com $0<\varepsilon<\varepsilon_{0}$ e defina os intervalos $I=\left(0, \varepsilon_{0}\right)$ e $J=\left(-\varepsilon_{0}, 0\right)$. Então, como $g(\varepsilon)=0$ e $g^{\prime}(\varepsilon)=2 \varepsilon \neq 0$, segue que $d_{B}\left(f^{0}+\varepsilon f^{1}+\varepsilon^{2} f^{2}, I, 0\right) \neq 0$. A mesma relação vale para $J$ no lugar de I. Logo, pelo Lema 1.5.10, se $\varepsilon$ é suficientemente pequeno $f(\cdot, \varepsilon)$ tem no mínimo duas raizes reais.

Teorema 1.5.15. (Averaging de primeira ordem via grau de Brouwer) Consideremos o seguinte sistema diferencial

$$
\dot{x}=\varepsilon f^{1}(x, t)+\varepsilon^{2} f^{2}(x, t, \varepsilon),
$$

onde $f^{1}: D \times \mathbb{R} \rightarrow \mathbb{R}^{n}, f^{2}: D \times \mathbb{R} \times\left(-\varepsilon_{f}, \varepsilon_{f}\right) \rightarrow \mathbb{R}^{n}$ são funções contínuas, T-periódicas em $t$ e $D$ é um subconjunto aberto de $\mathbb{R}^{n}$. Definimos $f: D \rightarrow \mathbb{R}^{n}$ como

$$
f(z)=\int_{0}^{T} f^{1}(z, s) d s
$$

e assumamos que:

i) $f^{1}$ e $f^{2}$ são localmente Lipschitz com respeito a variável $x$;

ii) para $a \in D$ com $f(a)=0$, existe uma vizinhança $\Omega$ de a tal que $f(z) \neq 0$ para todo $z \in \bar{\Omega} \backslash\{a\}$ e $d_{B}(f, \Omega, 0) \neq 0$.

Então, para $|\varepsilon|>0$ suficientemente pequeno, existe uma solução T-periódica $\varphi(\cdot, \varepsilon)$ do sistema (1.5.4) tal que $\varphi(\cdot, \varepsilon) \rightarrow$ a quando $\varepsilon \rightarrow 0$.

Observação 1.5.16. A função $f$ definida no Teorema 1.5 .15 é chamada de função promediada. A palavra "promediado" deriva do castelhano e significa média, optamos pelo 
uso dela nessa dissertação por ser seu uso comum na literatura e devido ao contato com pesquisadores da área que fazem uso dela.

Antes de demonstrar o Teorema 1.5.15 vamos compreender a ideia que motivou seu uso no estudo das órbitas periódicas de um dado sistema diferencial. Considere um sistema na forma

$$
\dot{x}=f(x, t, \varepsilon)
$$

onde $f: D \times \mathbb{R} \times\left(-\varepsilon_{0}, \varepsilon_{0}\right) \rightarrow \mathbb{R}$ é uma função contínua, $T$-periódica em $t$, localmente Lipschitz e $D \subset \mathbb{R}^{n}$ é um subconjunto aberto e limitado. Considere, para cada $z \in D$, $x(z, \cdot, \varepsilon):\left[0, t_{z}\right) \rightarrow \mathbb{R}^{n}$ a solução dos sistema satisfazendo $x(z, 0, \varepsilon)=z$.

Afirmamos que $t_{z}>T$ para todo $z \in D$.

De fato, pelo Teorema da Existência e Unicidade, segue que existe um $t_{z}>h_{z}=$ $\inf \left(T, \frac{b}{M(\varepsilon)}\right), M(\varepsilon) \geq|f(x, t, \varepsilon)|$, para todo $t \in[0, T]$, para cada $x$ satisfazendo $|x-z| \leq b$ e para todo $z \in \bar{D}$. Se $|\varepsilon|$ é suficientemente pequeno, podemos escolher $M(\varepsilon)$ conveniente de forma que $h_{z}=T$, para todo $z \in \bar{D}$, o que mostra a afirmação.

Considere agora a função $\bar{f}: D \times\left(-\varepsilon_{f}, \varepsilon_{f}\right) \rightarrow \mathbb{R}^{n}$ dada por:

$$
\bar{f}(z, \varepsilon)=\int_{0}^{T} f(x(z, s, \varepsilon), s, \varepsilon) d s
$$

Como $f$ é $T$-periódica e temos $t_{z}>T$, cada solução $x:[0, T] \rightarrow \mathbb{R}^{n}$ satisfazendo $x(0)=$ $x(T)$ pode ser extendida a $\mathbb{R}$ devido à sua periodicidade. Por outro lado, vale a seguinte relação

$$
\bar{f}(z, \varepsilon)=\int_{0}^{T} f(x(z, s, \varepsilon), s, \varepsilon) d s=\int_{0}^{T} \frac{d x}{d s}(z, s, \varepsilon) d s=(x(z, T, \varepsilon)-x(z, 0, \varepsilon))
$$

Sendo assim, se $x(z, \cdot, \varepsilon)$ é uma solução periódica do sistema (1.5.6), então a função $\bar{f}(z, \varepsilon)$ possui um zero. A recíproca também é verdadeira, isto é, se $\bar{f}(z, \varepsilon)=0$, temos $x(z, T, \varepsilon)=x(z, 0, \varepsilon)$. Ou seja, cada ponto singular $\left(z_{\varepsilon}, \varepsilon\right)$ de $\bar{f}$ fornece uma solução periódica $x\left(z_{\varepsilon}, \cdot, \varepsilon\right)$ de $\dot{x}=f(x, t, \varepsilon)$.

Dessa maneira, o problema de encontrar soluções $T$-periódicas de um sistema pode ser substituído pelo de encontrar zeros de uma certa função definida em um espaço de 
dimensão finita. Percebemos assim onde a teoria do grau de Brouwer se encaixa.

Se $f$ é de classe $C^{k}$ em $\varepsilon$, podemos escrevê-la usando sua série de Mac Laurin

$$
f(z, \varepsilon)=f(z, 0)+\varepsilon \frac{\partial f}{\partial \varepsilon}(z, 0)+\cdots+\varepsilon^{k} \frac{1}{k !} \frac{\partial f}{\partial \varepsilon}(z, 0)+\varepsilon^{k+1} r(z, \varepsilon) .
$$

Defina

$$
g(z, \varepsilon)=\sum_{i=0}^{k} \varepsilon^{i} \frac{1}{i !} \frac{\partial f}{\partial \varepsilon^{i}}(z, 0)
$$

e escreva

$$
f(z, \varepsilon)=g(z, \varepsilon)+\varepsilon^{k+1} r(z, \varepsilon) .
$$

Note que a função $f$ está escrita no formato do Lema 1.5.10. Se $\varepsilon \neq 0$, então podemos escrever $r(z, \varepsilon)=\frac{f(z, \varepsilon)-g(z, \varepsilon)}{\varepsilon^{k+1}}$ e então $r(z, \varepsilon)$ será contínua em $D \times\left(\left(-\varepsilon_{0}, \varepsilon_{0}\right) \backslash\{0\}\right)$. Em particular, se $K \subset D$ é um compacto, então $r$ será contínua em $K \times\left(\left(-\varepsilon_{0}, \varepsilon_{0}\right) \backslash\{0\}\right)$, portanto $r$ fica limitada em $K \times\left[-\varepsilon_{0}, \varepsilon_{0}\right]$ para $\varepsilon \neq 0$ e temos

$$
f(z, \varepsilon)=g(z, \varepsilon)+\varepsilon^{k+1} \mathcal{O}(1)
$$

Agora estamos preparados para demonstrar o teorema do averaging de primeira ordem.

Demonstração: Para todo $z \in \bar{\Omega}$, existe $\varepsilon_{0}>0$ tal que, se $\varepsilon \in\left[-\varepsilon_{0}, \varepsilon_{0}\right]$, então $x(z, \cdot, \varepsilon)$ está definida em $[0, T]$. Assim, para cada $t \in[0, T], z \in \bar{\Omega}$ e $\varepsilon \in\left[-\varepsilon_{0}, \varepsilon_{0}\right]$, vale a seguinte relação

$$
x(z, t, \varepsilon)=z+\varepsilon \int_{0}^{t} f^{1}(x(z, s, \varepsilon), s, \varepsilon) d s+\varepsilon^{2} \int_{0}^{t} f^{2}(x(z, s, \varepsilon), s, \varepsilon) d s .
$$

Neste caso, a função $\bar{f}$ se escreve como

$$
\bar{f}(z, \varepsilon)=\varepsilon \int_{0}^{T} f^{1}(x(z, s, \varepsilon), s) d s+\varepsilon^{2} \int_{0}^{T} f^{2}(x(z, s, \varepsilon), s, \varepsilon) d s .
$$

\section{Afirmação 1.5.17.}

$$
\bar{f}(z, \varepsilon)=\varepsilon f(z)+\mathcal{O}\left(\varepsilon^{2}\right) e m \bar{\Omega} \times\left[-\varepsilon_{0}, \varepsilon_{0}\right],
$$


onde $f(z)$ é dada por (1.5.5).

De fato, pelo que foi discutido anteriormente, existe um compacto $K \subset D$ tal que $x(z, t, \varepsilon) \in K$, para todo $t \in[0, T], z \in \bar{\Omega}$, e $\varepsilon \in\left[-\varepsilon_{0}, \varepsilon_{0}\right]$. Logo, como $f^{2}$ é contínua em $[0, T] \times\left[-\varepsilon_{0}, \varepsilon_{0}\right]$, existe um $N_{K}>0$ tal que $\left|f^{2}(x(z, t, \varepsilon), t, \varepsilon)\right| \leq N_{K}$. Assim

$$
\left|\int_{0}^{T} f^{2}(x(z, s, \varepsilon), s, \varepsilon) d s\right| \leq \int_{0}^{T} N_{K} d s=N_{K} T=\mathcal{O}(1) .
$$

Por outro lado, temos

$$
\begin{aligned}
\varepsilon \int_{0}^{T} f^{1}(x(z, s, \varepsilon), s) d s & =\varepsilon \int_{0}^{T}\left[f^{1}(x(z, s, \varepsilon), s) d s-f^{1}(z, s)+f^{1}(z, s)\right] d s \\
& =\varepsilon \int_{0}^{T}\left[f^{1}(x(z, s, \varepsilon), s) d s-f^{1}(z, s)\right] d s+\varepsilon \int_{0}^{T} f^{1}(z, s) d s
\end{aligned}
$$

Sendo assim,

$$
\begin{aligned}
\bar{f}(z, \varepsilon)-\varepsilon f(z) & =\varepsilon \int_{0}^{T} f^{1}(x(z, s, \varepsilon), s) d s+\varepsilon^{2} \mathcal{O}(1)-\varepsilon \int_{0}^{T} f^{1}(z, s) d s \\
& =\varepsilon \int_{0}^{T}\left[f^{1}(x(z, s, \varepsilon), s)-f^{1}(z, s)\right] d s+\varepsilon^{2} \mathcal{O}(1) .
\end{aligned}
$$

Como $f^{1}$ é Lipschitz na variável $x$ em $[0, T] \times K$ e a solução $x(z, t, \varepsilon)$ se mantém em $K \subset D$, temos

$$
|\bar{f}(z, \varepsilon)-\varepsilon f(z)| \leq \varepsilon \int_{0}^{T}|x(z, s, \varepsilon)-z| L_{K} d s=\mathcal{O}\left(\varepsilon^{2}\right)
$$

Logo,

$$
\bar{f}(z, \varepsilon)-\varepsilon f(z)=\mathcal{O}\left(\varepsilon^{2}\right) \Rightarrow \bar{f}(z, \varepsilon)=\varepsilon f(z)+\mathcal{O}\left(\varepsilon^{2}\right)
$$

completando a demonstração da afirmação.

Pela hipótese $(i i)$ podemos aplicar o Corolário 1.5.13 para garantir que existe $z_{\varepsilon}$ tal que $\bar{f}\left(z_{\varepsilon}, \varepsilon\right)=0$ e $z_{\varepsilon} \rightarrow a$ quando $\varepsilon \rightarrow 0$. Portanto, $\varphi(\cdot, \varepsilon)=x\left(z_{\varepsilon}, \cdot, \varepsilon\right)$ é uma solução periódica de (1.5.4) e $\varphi(\cdot, \varepsilon) \rightarrow a$ quando $\varepsilon \rightarrow 0$.

Definição 1.5.18. Seja $x_{0} \in D$ e $x\left(x_{0}, t, \varepsilon\right)$ a solução de (1.5.4), satisfazendo $x\left(x_{0}, 0, \varepsilon\right)=$ 
$x_{0}$. Dada uma vizinhança $U \subset D$ de $x_{0}$ definimos a aplicação $P_{\varepsilon}: U \rightarrow D$, conhecida como mapa de Poincaré e dada por

$$
P_{\varepsilon}(z)=x(z, T, \varepsilon)
$$

Note que $P_{\varepsilon}$ está bem definida para $|\varepsilon|$ suficientemente pequeno, uma vez que a solução $x(z, t, \varepsilon)$ pode ser estendida para $[0, T]$.

Corolário 1.5.19. O mapa de Poincaré $P_{\varepsilon}(z)$, definido acima para $\varepsilon$ suficientemente pequeno, é dado por

$$
P_{\varepsilon}(z)=z+\varepsilon f(z)+\mathcal{O}\left(\varepsilon^{2}\right)
$$

Demonstração: Pela demonstração do Teorema 1.5.15, temos as seguintes igualdades

$$
\begin{aligned}
& x(z, T, \varepsilon)=z+\bar{f}(z, \varepsilon), \\
& \bar{f}(z, \varepsilon)=\varepsilon f(z)+\mathcal{O}\left(\varepsilon^{2}\right) .
\end{aligned}
$$

Logo

$$
x(z, T, \varepsilon)=z+\varepsilon f(z)+\mathcal{O}\left(\varepsilon^{2}\right)
$$

O próximo exemplo ilustrará como aplicar o teorema do averaging no caso contínuo.

Exemplo 1.5.20. Considere a equação diferencial planar, conhecida como sistema de Lienard,

$$
\left\{\begin{array}{l}
\dot{x}=y, \\
\dot{y}=-x-\varepsilon(h(x)+g(x)),
\end{array}\right.
$$

onde

$$
h(x)=\sum_{i=0}^{7} a_{i} x^{i} \quad e \quad g(x)=\sum_{i=0}^{4} b_{i} x^{i}
$$

Observe primeiramente que o sistema não está na forma padrão, mas escrevendo $x=$ 
$r \cos \theta$ e $y=r \operatorname{sen} \theta$, com $r>0$, o novo sistema obtido é dado por

$$
\left\{\begin{array}{l}
\dot{r}=-\varepsilon\left(\sum_{i=0}^{7} a_{i} r^{i+1} \cos ^{i} \theta \operatorname{sen}^{2} \theta+\sum_{i=0}^{4} b_{i} r^{i} \cos ^{i} \theta \operatorname{sen} \theta\right) \\
\dot{\theta}=-1-\frac{\varepsilon}{r}\left(\sum_{i=0}^{7} a_{i} r^{i+1} \cos ^{i+1} \theta \operatorname{sen} \theta+\sum_{i=0}^{4} b_{i} r^{i} \cos ^{i+1} \theta\right) .
\end{array}\right.
$$

Tomando $\theta$ como a nova variável independente, reescrevemos o sistema original como

$$
\frac{d r}{d \theta}=\varepsilon\left(\sum_{i=0}^{7} a_{i} r^{i+1} \cos ^{i} \theta \operatorname{sen}^{2} \theta+\sum_{i=0}^{4} b_{i} r^{i} \cos ^{i} \theta \operatorname{sen} \theta\right)+\mathcal{O}\left(\varepsilon^{2}\right)
$$

que agora está na forma padrão. Nesse caso, a função promediada é dada por

$$
f(r)=\int_{0}^{2 \pi}\left(\sum_{i=0}^{7} a_{i} r^{i+1} \cos ^{i} \theta \operatorname{sen}^{2} \theta+\sum_{i=0}^{4} b_{i} r^{i} \cos ^{i} \theta \operatorname{sen} \theta\right) d \theta .
$$

Para calcular essa integral, faremos uso das seguintes expressões válidas para $k=$ $0,1,2, \ldots$

$$
\begin{gathered}
\int_{0}^{2 \pi} \cos ^{2 k+1} \operatorname{sen}^{2} \theta d \theta=0, \\
\int_{0}^{2 \pi} \cos ^{2 k} \operatorname{sen}^{2} \theta d \theta=\alpha_{2 k} \neq 0, \\
\int_{0}^{2 \pi} \cos ^{k} \operatorname{sen} \theta d \theta=0,
\end{gathered}
$$

Assim,

$$
f(r)=2 \pi\left(\frac{a_{0}}{2} r+\frac{a_{2}}{8} r^{3}+\frac{a_{4}}{16} r^{5}+\frac{5 a_{6}}{128} r^{7}\right) .
$$

Logo $f(0)=0$ e $f$ é uma função ímpar, assim, se $r_{0}$ é raiz de $f,-r_{0}$ também será. Sendo assim, a função tem no máximo 3 raízes positivas e segue do Teorema 1.5.15 que a equação de Lienard (1.5.7) possui exatamente três ciclos limites bifurcando-se do centro linear $\dot{x}=y, \dot{y}=-x$. 


\subsubsection{Averaging períodico de segunda ordem via grau de Brouwer}

Quando a hipótese (ii) do Teorema 1.5.15 não for verificada, devemos usar a técnica do averaging de segunda ordem, cuja prova é muito semelhante a de primeira ordem e pode ser encontrada em [11], abaixo seu enunciado é apresentado.

Teorema 1.5.21. Considere o seguinte sistema diferencial

$$
\dot{x}(t)=\varepsilon f^{1}(x, t)+\varepsilon^{2} f^{2}(x, t)+\varepsilon^{3} f^{3}(x, t, \varepsilon),
$$

onde $f^{1}, f^{2}: D \times \mathbb{R} \rightarrow \mathbb{R}^{n}$ e $f^{3}: D \times \mathbb{R} \times\left(\varepsilon_{f}, \varepsilon_{f}\right) \rightarrow \mathbb{R}^{n}$ são funções contínuas, T-periódicas em $t$ e $D$ é um aberto de $\mathbb{R}^{n}$. Defina $\bar{f}^{1}, \bar{f}^{2}: D \rightarrow \mathbb{R}^{n}$ por

$$
\begin{gathered}
\bar{f}^{1}(z)=\int_{0}^{T} f^{1}(z, s) d s \\
\bar{f}^{2}(z)=\int_{0}^{T}\left[D_{z} f^{1}(z, s) \cdot \int_{0}^{s} f^{1}(z, t) d t+f^{2}(z, s)\right] d s
\end{gathered}
$$

e assuma que

i) $f^{1}(\cdot, t) \in C^{1}(D) \quad \forall t \in \mathbb{R}, f^{1}, f^{2}, f^{3}$ e $D_{x} f^{1}$ sejam localmente Lipschitz com respeito à variável $x$ e $f^{3}$ seja diferenciável com respeito a $\varepsilon$.

ii) para $a \in D$ tal que $\bar{f}^{1}(a)+\varepsilon \bar{f}^{2}(a)=0$, existe uma vizinhança $\Omega$ de a tal que $\bar{f}^{1}(z)+\varepsilon \bar{f}^{2}(z) \neq 0$ para todo $z \in \bar{\Omega} \backslash\{a\}$ e $d_{B}\left(\bar{f}^{1}+\varepsilon \bar{f}^{2}, \Omega, 0\right) \neq 0$.

Então, para $|\varepsilon|>0$ suficientemente pequeno, existe uma solução T-periódica $\varphi(\cdot, \varepsilon)$ do sistema (1.5.8) tal que $\varphi(\cdot, \varepsilon) \rightarrow$ a quando $\varepsilon \rightarrow 0$.

Exemplo 1.5.22. (Equação de Van der Pol modificada) Considere o sistema

$$
\left\{\begin{array}{l}
\dot{x}=y, \\
\dot{y}=-x+\varepsilon x^{2}+\varepsilon^{2}\left(1-x^{2}\right) y .
\end{array}\right.
$$


Seja $x=r \operatorname{sen} \theta$ e $y=r \cos \theta$. Então o sistema (1.5.9) é reescrito como

$$
\left\{\begin{array}{l}
\dot{r}=\varepsilon r^{2} \operatorname{sen}^{2} \theta \cos \theta+\varepsilon^{2}\left(1-r^{2} \operatorname{sen}^{2} \theta\right) r \cos ^{2} \theta, \\
\dot{\theta}=1-\varepsilon r \operatorname{sen}^{3} \theta-\varepsilon^{2}\left(1-r^{2} \operatorname{sen}^{2} \theta\right) \cos \theta \operatorname{sen} \theta .
\end{array}\right.
$$

Tomando $\theta$ como a nova variável independente obtemos

$$
\frac{d r}{d \theta}=\varepsilon r^{2} \operatorname{sen}^{2} \theta \cos \theta+\varepsilon^{2}\left(1-r^{2} \operatorname{sen}^{2} \theta\right) r \cos ^{2} \theta+\mathcal{O}\left(\varepsilon^{3}\right)
$$

Identificando o sistema acima(conferir Teorema 1.5.21) temos

$$
f^{1}(r, \theta)=r^{2} \operatorname{sen}^{2} \theta \cos \theta \quad \text { e } \quad f^{2}(r, \theta)=\left(1-r^{2} \operatorname{sen}^{2} \theta\right) r \cos ^{2} \theta
$$

Assim, $D_{r} f^{1}(r, \theta)=2 r \operatorname{sen}^{2} \theta \cos \theta, \overline{f^{1}}(r)=\int_{0}^{2 \pi} r^{2} \operatorname{sen}^{2} \theta \cos \theta=0 \mathrm{e}$

$$
\begin{aligned}
\overline{f^{2}}(r) & =\int_{0}^{2 \pi}\left[D_{r} f^{1}(r, \theta) \int_{0}^{\theta} f^{1}(r, s) d s+f^{2}(r, \theta)\right] d \theta \\
& =\int_{0}^{2 \pi} \frac{2}{3} r \operatorname{sen}^{2} \theta \cos \theta \operatorname{sen}^{3} \theta+\left(1-r^{2} \operatorname{sen}^{2} \theta\right) r \cos ^{2} \theta d \theta \\
& =\pi\left(r-\frac{r^{3}}{4}\right)
\end{aligned}
$$

Note que as raízes de $\overline{f^{2}}(r)=0$ são $-2,0$ e 2 e como ${\overline{f^{2}}}^{\prime}(2)=\pi\left(1-\frac{3.4}{4}\right)=-2 \pi \neq 0$ segue do Teorema 1.5.21 que o sistema (1.5.9) possui uma órbita periódica $\varphi_{\varepsilon}(t)$ de período próximo a $2 \pi$ de tal forma que $\varphi_{\varepsilon}(t)$ tende ao círculo centrado na origem de raio $\sqrt{2}$ quando $\varepsilon$ tende a zero. 


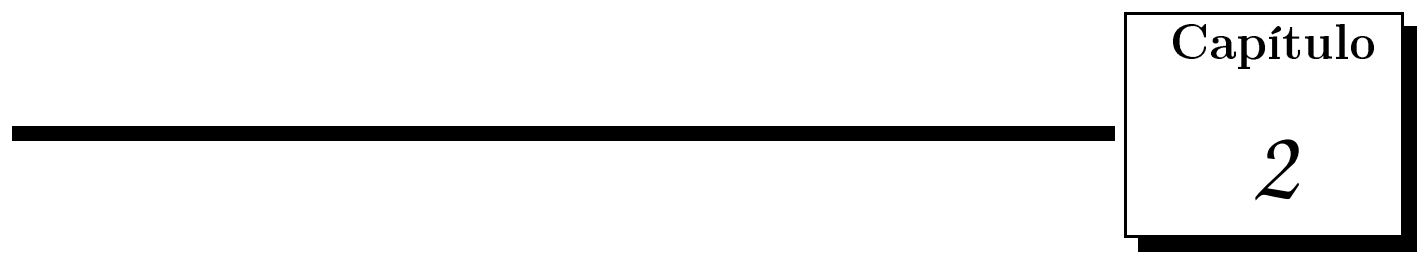

\section{Sistemas de Filippov}

Neste capítulo vamos introduzir o conceito de sistema de Filippov e investigar as semelhanças e diferenças entre esses sistemas e os sistemas diferenciais suaves.

Considere o sistema contínuo por partes

$$
X(x)= \begin{cases}-1 & \text { se } x>0 \\ 1 & \text { se } x \leq 0 .\end{cases}
$$

O único ponto de descontinuidade do sistema ocorre para $x=0$ e não é possível encontrar uma solução $\gamma:\left[0, t_{1}\right] \rightarrow \mathbb{R}$ continuamente diferenciável que satisfaça a relação $\dot{\gamma}(t)=$ $X(\gamma(t))$ e $\gamma(0)=0$.

De fato, se tal solução existisse, teríamos $\dot{\gamma}(0)=X(\gamma(0))=X(0)=1$ e $\dot{\gamma}(t)=-1$, para qualquer $t$ suficientemente próximo de zero, o que contradiz o fato de $\dot{\gamma}$ ser contínua. Com esse exemplo bem simples, vemos que a descontinuidade em $x=0$ leva a não existência de solução contínuamente diferenciável do sistema.

Assim concluímos que para a investigação dos sistemas com descontinuidades as definições precisam ser modificadas.

Seja $D \subset \mathbb{R}^{n}$ um subconjunto aberto conexo e $h: \mathbb{R} \times D \rightarrow \mathbb{R}$ uma função de classe $C^{1}$ tendo $0 \in \mathbb{R}$ como valor regular e considere $X, Y: \mathbb{R} \times D \rightarrow \mathbb{R}^{n}$ funções contínuas. 
Seja $\Sigma=h^{-1}(0)$ e defina o sistema de Filippov

$$
\dot{x}(t)=Z(t, x)=\left\{\begin{array}{l}
X(t, x),(t, x) \in \Sigma^{+}, \\
Y(t, x),(t, x) \in \Sigma^{-},
\end{array}\right.
$$

onde,

$$
\begin{aligned}
& \Sigma^{+}=\{(t, x) \in \mathbb{R} \times D: h(t, x)>0\}, \\
& \Sigma^{-}=\{(t, x) \in \mathbb{R} \times D: h(t, x)<0\} .
\end{aligned}
$$

Nesse caso, usamos a notação $Z=(X, Y)$.

Na literatura são usuais os termos "sistema descontínuo" e "sistema suave por partes" quando nos referimos a um sistema de Filippov.

\subsection{Trajetória local e singularidades}

Dado que nosso objetivo é investigar possíveis extensões dos conceitos conhecidos para sistemas diferenciais suaves para a classe dos sistemas diferenciais com descontinuidades, parece natural definir que para $p \in \Sigma^{+}$ou $\Sigma^{-}$a trajetória local pelo ponto $p$ como a trajetória do sistema correspondente, enquanto que os pontos em $\Sigma$ são divididos em três regiões disjuntas, a saber

(i) região de costura: $\Sigma^{c}=\{p \in \Sigma: X h(p) . Y h(p)>0\}$,

(ii) região de deslize: $\Sigma^{s}=\{p \in \Sigma: X h(p)<0$ e $Y h(p)>0\}$,

(iii) região de escape: $\Sigma^{e}=\{p \in \Sigma: X h(p)>0$ e $Y h(p)<0\}$.

Definição 2.1.1. Um ponto $p \in \Sigma$ é chamado ponto de tangência se $X h(p)=0$ ou $Y h(p)=0$

Os pontos de tangência podem ser classificados de acordo com seu contato com a região de descontinuidade $\Sigma$.

Definição 2.1.2. Um campo vetorial suave $X$ possui uma dobra ou tangência quadrática com $\Sigma$ em um ponto $p$ se $X h(p)=0$ e $X^{2} h(p) \neq 0$. 
Definição 2.1.3. Um campo vetorial suave $X$ possui uma cúspide ou tangência cúbica com $\Sigma$ em um ponto $p$ se $X h(p)=X^{2} h(p)=0$ e $X^{3} h(p) \neq 0$.

Afim de simplificar a definição de órbita que será introduzida a seguir, assumimos que todos os pontos de tangência em $\Sigma$ são isolados.

\section{$\operatorname{Em} \Sigma^{c}$}

Na região de costura os campos apontam para a mesma direção e portanto definimos a trajetória de $Z$ como a justaposição das trajetórias de $X$ e $Y$ pelo ponto $p \in \Sigma$, como mostra a Figura 2.1.

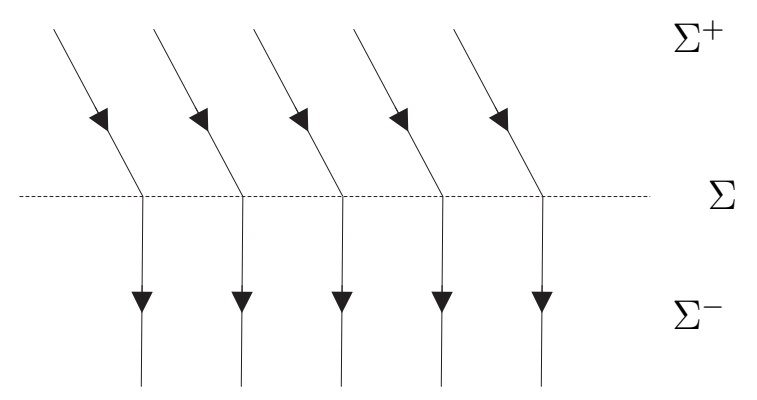

Figura 2.1: Região de costura

\section{$\underline{\operatorname{Em} \Sigma^{s} \text { e } \Sigma^{e}}$}

Para essas regiões, considere o campo auxiliar, denotado por $Z^{s}$ e chamado de campo vetorial deslizante, dado pela seguinte combinação linear convexa de $X(p)$ e $Y(p)$

$$
Z^{s}(p)=\frac{1}{Y h(p)-X h(p)}(Y h(p) X(p)-X h(p) Y(p))
$$

Observe que o campo $Z^{s}$ é tangente à $\Sigma$ em $p$. Uma representação geométrica para $Z^{s}$ é dada na Figura 2.2.

Definição 2.1.4. Para $p \in \Sigma^{s} \cup \Sigma^{e}$, a trajetória local de $p$ é dada pelo campo vetorial $Z^{s}(p)$. Ou seja, considerando p como um ponto em que o campo contínuo $Z^{s}$ está definido, calculamos a trajetória local passando por $p$.

Em resumo 


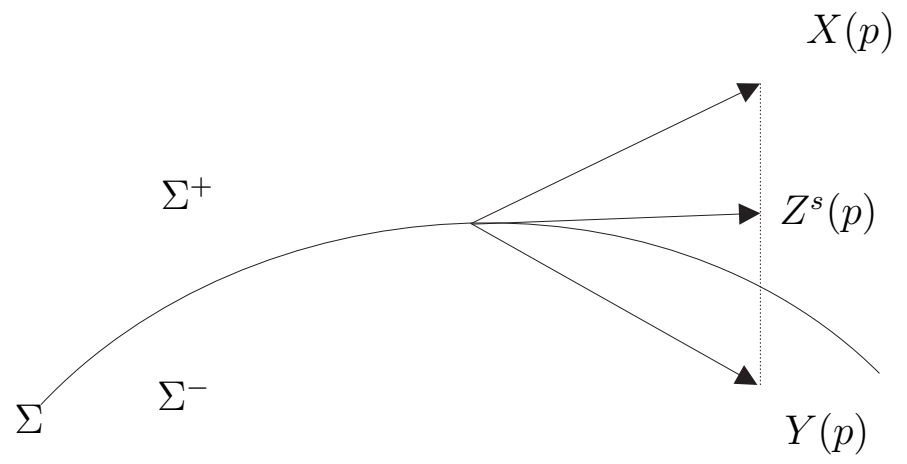

Figura 2.2: Campo vetorial deslizante

i) Para $p \in \Sigma^{+}$tal que $X(p) \neq 0$ (pontos regulares de $X$ em $\Sigma^{+}$) a trajetória passando por $p$ é definida como sendo $\varphi_{Z}(t, p)=\varphi_{X}(t, p)$, para $t \in I \subset \mathbb{R}$. Analogamente para os pontos regulares de $Y$ em $\Sigma^{-}$.

ii) Para $p \in \Sigma^{c}$ tal que $X h(p), Y h(p)>0$ e tomando a origem do tempo em $p$, a trajetória é definida como $\varphi_{Z}(t, p)=\varphi_{Y}(t, p)$, para $t \in I_{Y} \cap(-\infty, 0]$ e $\varphi_{Z}(t, p)=$ $\varphi_{X}(t, p)$, para $t \in I_{X} \cap[0, \infty)$. Para o caso $X h(p), Y h(p)<0$ a definição é análoga com o tempo com sinal oposto.

iii) Para $p \in \Sigma^{e} \cup \Sigma^{s}$ tal que $Z^{s}(p) \neq 0, \varphi_{Z}(t, p)=\varphi_{Z^{s}}(t, p)$, para $t \in \mathbb{R}$.

iv) Para $p \in \partial \Sigma^{c} \cup \partial \Sigma^{s} \cup \partial \Sigma^{e}$ tal que as definições de trajetórias para pontos em $\Sigma$ em ambos os lados de $p$ podem ser estendidas para $p$ e coincidem, a trajetória pelo ponto é exatamente essa extensão. A estes pontos damos o nome de tangência regulares.

Exemplo 2.1.5. Considere o sistema

$$
Z_{1}(x, y)=\left\{\begin{array}{l}
X=\left(\begin{array}{c}
1 \\
x^{2}
\end{array}\right) \quad \text { se } y>0 \\
Y=\left(\begin{array}{c}
1 \\
1
\end{array}\right) \quad \text { se } y<0
\end{array}\right.
$$

e seja $p=(0,0) \in \Sigma=\{(x, y) ; y=0\}$. O retrato de fase desse sistema é dado na Figura 2.3. Nesse caso, a origem é um ponto de tangência regular. 


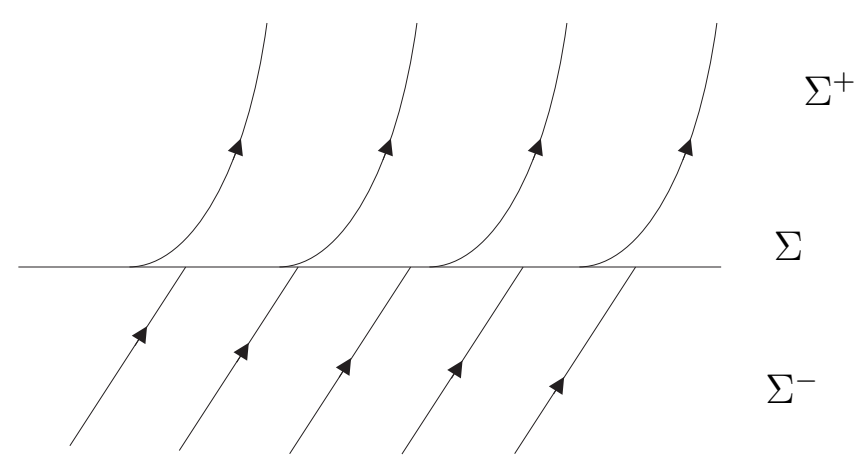

Figura 2.3: Retrato de fase do campo $Z_{1}$

v) Para pontos que não foram contemplados nos itens anteriores acima definimos $\varphi_{Z}(t, p)=\{p\}$. Aqui, estão os pontos de tangência não regulares, chamados tangências singulares, os pontos críticos de $X$ e $Y$ em $\Sigma^{ \pm}$e os pontos críticos do campo deslizante $Z^{s}$, em $\partial \Sigma^{e} \cup \partial \Sigma^{s}$

Definição 2.1.6. Seja $Z: \mathbb{R} \times D \rightarrow \mathbb{R}^{n}, Z=(X, Y)$ um campo de vetores suave por partes. A órbita local de um ponto $p \in D$ é o conjunto $\gamma(p)=\left\{\varphi_{Z}(t, p) ; t \in I\right\}$, onde $\varphi_{Z}$ está dada pela Definição 2.1.4.

No que segue, daremos uma série de definições afim de generalizar conceitos conhecidos para sistemas suaves.

Definição 2.1.7. As singularidades do sistema de Filippov são caracterizadas pelos seguintes tipos

(i) $p \in \Sigma^{ \pm}$tal que $p$ é singularidade de $X$ ou de $Y$;

(ii) $p \in \Sigma^{s} \cup \Sigma^{e}$ tal que $Z^{s}(p)=0$;

(iii) $p \notin\left(\Sigma^{+} \cup \Sigma^{-} \cup \Sigma^{c} \cup \Sigma^{s} \cup \Sigma^{e}\right)$, isto é, os pontos $p$ tais que $X h(p)=0$ ou $Y h(p)=0$.

Qualquer outro ponto é chamado de ponto regular do sistema $Z=(X, Y)$.

Observação 2.1.8. Em sistemas diferenciais suaves, as trajetórias em pontos críticos são o próprio ponto. Entretanto, para sistemas de Filippov, existem singularidades, como as tangências regulares, cujas órbitas são tais que $\gamma \nsupseteq\{p\}$. Por esta razão dizemos que uma singularidade é 
a) distinguida: quando $p$ é tal que $\gamma(p)=\{p\}$.

b) não distinguida: quando $p \in \Sigma$ é ponto de tangência regular. Nesse caso a órbita por $p$ é homeomorfa a $\mathbb{R}$.

Definição 2.1.9. Dados uma trajetória $\varphi_{Z}(t, q) \in \Sigma^{+} \cup \Sigma^{-}$e um ponto $p \in D$, dizemos que $p$ é um ponto de partida de $\varphi_{Z}(t, q)$ se existe $t_{0}<0$ tal que

$$
\lim _{t \rightarrow t_{0}-} \varphi_{Z}(t, q)=p
$$

e p é um ponto de chegada de $\varphi_{Z}(t, q)$ se existe $t_{0}>0$ tal que

$$
\lim _{t \rightarrow t_{0}+} \varphi_{Z}(t, q)=p
$$

De acordo com a definição de órbita se $p \in \Sigma^{c}$ então $p$ é um ponto de partida de $\varphi_{Z}(t, q)$ para qualquer $q$ pertencendo à órbita $\gamma^{+}(p)=\left\{\varphi_{Z}(t, q) ; t \in I \cap[0, \infty)\right\}$, e é um ponto de chegada de $\varphi_{Z}(t, q)$ para qualquer $q$ pertencendo à órbita $\gamma^{-}(p)=\left\{\varphi_{Z}(t, q) ; t \in\right.$ $I \cap(-\infty, 0]\}$. Assim, a órbita passando por um ponto $p \in \Sigma^{c}$ é a união do ponto com suas órbitas de partida e chegada, isto é, $\gamma(p)=\{p\} \cup \gamma^{+}(p) \cup \gamma^{-}(p)$. Note que esse fato reforça a ideia intuitiva de órbitas por pontos $p$ na região de costura.

\section{Classificação das singularidades distinguidas}

As singularidades distinguidas podem ser classificados em três grupos. Veremos que cada grupo apresenta um comportamento distinto, embora todos tenham algo em comum pois a órbita do sistema pela singularidade distinguida é somente o ponto.

Grupo 1: pontos de $\partial \Sigma^{c}$ que não são pontos de chegada nem de partida de trajetórias. Para pontos nesse grupo o retrato de fase local é similar ao de um foco em sistemas diferenciais suaves. 
Exemplo 2.1.10.

$$
Z_{2}(x, y)=\left\{\begin{array}{l}
X=\left(\begin{array}{c}
1 \\
-2 x
\end{array}\right) \quad \text { se } y>0, \\
Y=\left(\begin{array}{c}
-1 \\
-x+x^{2}
\end{array}\right) \text { se } y<0,
\end{array}\right.
$$

e $p=(0,0) \in \Sigma=\{(x, y) ; y=0\}$. O retrato de fase é dado pela Figura 2.4. Observe que as trajetórias de $Z_{2}$ espiralam em torno da origem como acontece em um foco.

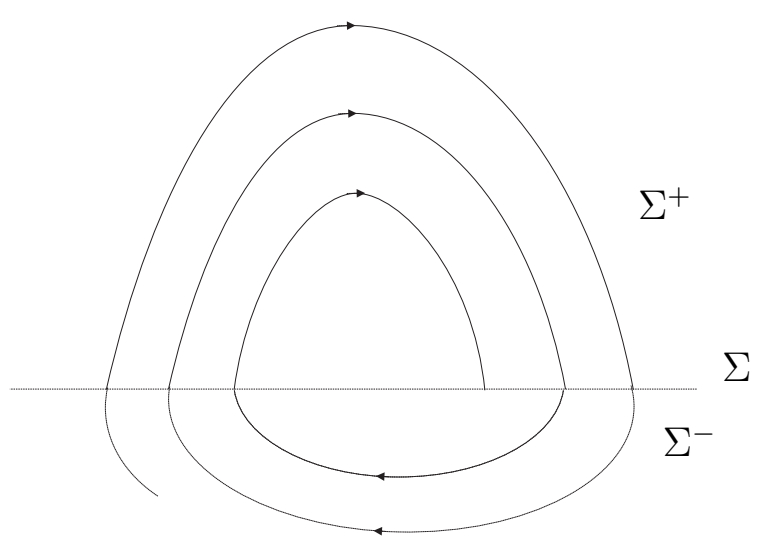

Figura 2.4: Retrato de fase do campo $Z_{2}$

Mostremos que p é de fato uma singularidade distinguida de $Z_{2}$

i) $p$ é singularidade: como $h(x, y)=y$ segue que $\nabla h(x, y)=(0,1)$ e assim $X h(p)=0$.

ii) a órbita passando por $p$ é $\{p\}$ : observe que as trajetórias de $X$ não podem ser estendidas à $p$ e portanto este ponto é realmente um ponto de singularidade distinguida de $Z$.

Grupo 2: pontos que pertencem a $\partial \Sigma^{c} \cap \partial \Sigma^{s}$ ou $\partial \Sigma^{c} \cap \partial \Sigma^{e}$. 
Considere os campos

$$
Z_{3}^{+}(x, y)=\left\{\begin{array}{l}
X=\left(\begin{array}{c}
1 \\
x
\end{array}\right) \text { se } y>0, \\
Y=\left(\begin{array}{c}
0 \\
y
\end{array}\right) \text { se } y<0 .
\end{array} \quad Z_{3}^{-}(x, y)=\left\{\begin{array}{c}
X=\left(\begin{array}{c}
-1 \\
x
\end{array}\right) \text { se } y>0, \\
Y=\left(\begin{array}{c}
0 \\
y
\end{array}\right)
\end{array}\right.\right.
$$

A variedade de descontinuidade é o eixo $0 x$ e $p=(0,0)$. Os retratos de fase de $Z_{3}{ }^{+}$e $Z_{3}{ }^{-}$ são dados na Figura 2.5. Observe que se $(x, 0) \in \Sigma$ é tal que $x<0$ então

$$
X h(x, 0)=x<0 \quad \text { e } \quad Y h(x, 0)=1>0 .
$$

Logo $(x, 0) \in \Sigma^{s}$. Para pontos tais que $(x, 0) \in \Sigma$ e $x>0$, temos

$$
X h(x, 0)=x>0 \quad \text { e } \quad Y h(x, 0)=1>0 .
$$

Portanto $(x, 0) \in \Sigma^{c}$. Assim, $p \in \partial \Sigma^{c} \cap \partial \Sigma^{s}$ e para pontos à esquerda de $p$, suas órbitas são dadas pelo campo deslizante $Z^{s}$. Já para pontos à direita de $p$, a trajetória é dada pela reunião das trajetórias em $X$ e $Y$ (região de costura). Sendo assim $p$ é uma tangência singular, tanto para $Z_{3}^{+}$quanto para $Z_{3}^{-}$.
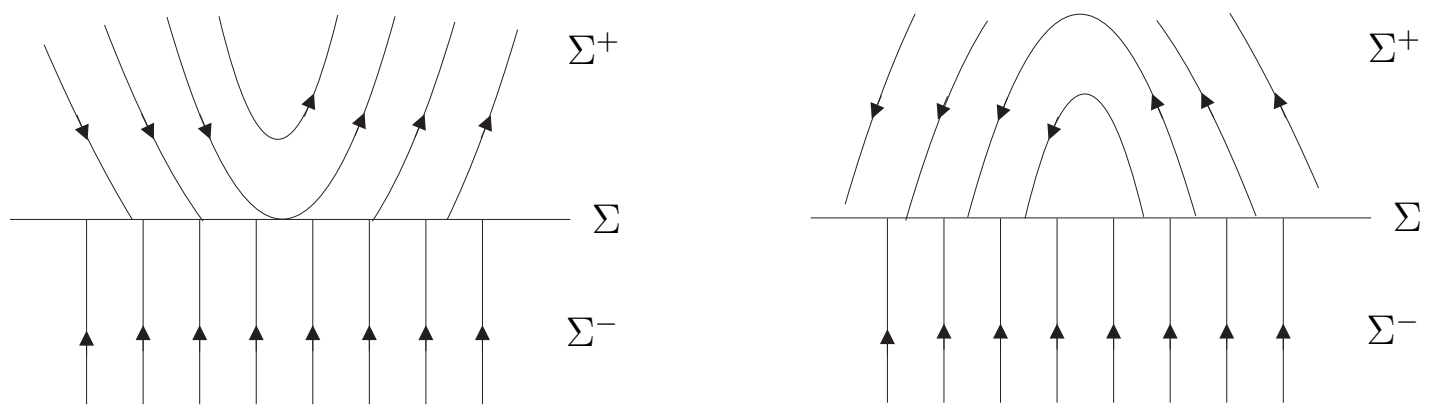

Figura 2.5: Retratos de fase dos campos $Z_{3}^{+}$e $Z_{3}^{-}$

Grupo 3: pontos de tangência singulares em $\partial \Sigma^{c}$ que são pontos de partida ou chegada de duas trajetórias diferentes de $X$ e $Y$.

Note que para esses pontos, como diferentes trajetórias de $X$ e $Y$ partem(ou chegam) 
dele(a ele), a única escolha que nos garante unicidade de soluções é considerar o próprio ponto como sua órbita. Vejamos um exemplo.

Exemplo 2.1.11. Considere o campo

$$
Z_{4}(x, y)=\left\{\begin{array}{l}
X=\left(\begin{array}{c}
1 \\
x
\end{array}\right) \quad \text { se } y>0 \\
Y=\left(\begin{array}{c}
-1 \\
x
\end{array}\right) \text { se } y<0
\end{array}\right.
$$

a curva $\Sigma$ como sendo o eixo $O x$ e $p=(0,0)$. O seu retrato de fase é dado pela Figura 2.6. Neste caso, a origem é um ponto de dobra visivel para X e Y. Assim, teremos um par de órbitas para os quais p é um ponto de chegada e outro par para o qual a origem é um ponto de partida.

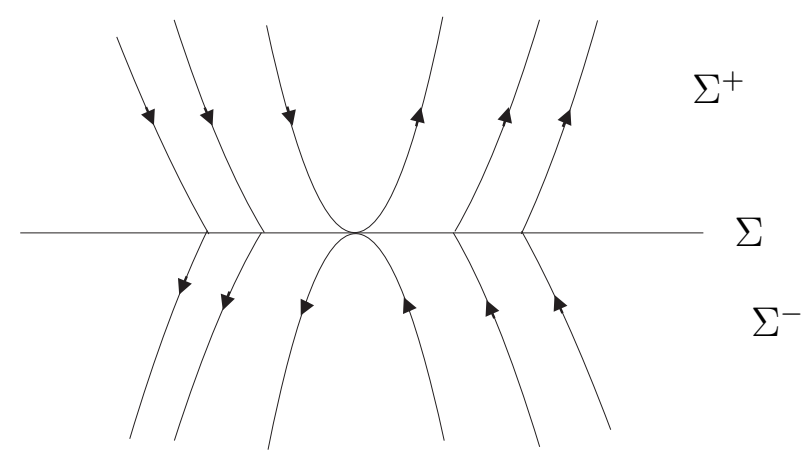

Figura 2.6: Retrato de fase do campo $Z_{4}$

Observação 2.1.12. Atualmente, tem-se discutido muito sobre o fato de considerar ou não que por um ponto passe uma única órbita quando estamos em uma situação parecida com a do exemplo anterior. Ao considerarmos o retrato de fase do campo $Z_{4}$ vemos que pela origem podemos ter pelo menos duas soluções: $A$ de $X$ e a de $Y$.

Do ponto de vista dinâmico, não é muito útil trabalhar com um ponto cuja órbita é trivial. Assim, tem-se permitido que a solução não seja única para que haja um leque maior de possibilidades de estudo do sistema. 
Agora que já temos a definição de órbita local, podemos nos perguntar o que seria uma órbita regular maximal passando por um ponto. A resposta vem com a seguinte definição

Definição 2.1.13. Uma órbita regular de $Z$ é uma curva $\gamma$ suave por partes tal que

(i) $\gamma \cap \Sigma^{+}$e $\gamma \cap \Sigma^{-}$é uma união de órbitas dos campos $X$ e $Y$, respectivamente.

(ii) A interseção $\gamma \cap \Sigma$ consiste apenas de pontos de costura e de tangências regulares em $\partial \Sigma^{c}$.

Uma órbita $\gamma$ é dita regular maximal se ela for maximal com respeito às condiçôes $(i)$ e (ii).

Definição 2.1.14. Uma órbita deslizante(ou órbita singular) maximal de $Z$ é uma curva suave $\gamma \subset \overline{\Sigma^{+}} \cup \overline{\Sigma^{-}}$que é uma órbita maximal do sistema suave $Z^{s}$.

\subsection{Separatrizes, órbitas periódicas e ciclos}

A ideia agora é generalizar os conceitos de separatrizes, órbitas periódicas e ciclos para campos de Filippov. No caso das separatrizes, elas também indicarão que há uma mudança de comportamento quando cruzamos de um lado para outro dela.

Definição 2.2.1. Seja $p \in D$ um ponto de sela para $X$ ou $Y$ em $\overline{\Sigma^{ \pm}}$ou uma singularidade distinguida em $\Sigma$.

i) Se $p \in \Sigma^{ \pm}$é um ponto de sela para $X$ em $\overline{\Sigma^{+}}$, então a separatriz instável de $p$ é o conjunto

$$
W^{u}(p) \doteq\left\{q \in D: \varphi_{Z}(t, q) \text { está definido para }(-\infty, 0) \text { e } \lim _{t \rightarrow-\infty} \varphi_{Z}(t, q)=p\right\}
$$

Analogamente para um ponto de sela $p \in \overline{\Sigma^{-}}$.

ii) Se $p \in \Sigma$ é uma singularidade distinguida, então a separatriz instável é uma órbita regular de $Z$ que possui $p \in \Sigma$ como ponto de partida.

Denotaremos essa separatriz por $W_{ \pm}^{u}(p)$, onde o subescrito \pm significa que a órbita está contida em $\Sigma^{ \pm}$. 
Definição 2.2.2. A separatriz estável é definida de maneira análoga, usando a notação

$$
W^{s}(p) \doteq\left\{q \in D: \varphi_{Z}(t, q) \text { está definido para }(0, \infty) \text { e } \lim _{t \rightarrow \infty} \varphi_{Z}(t, q)=p\right\}
$$

para pontos de sela para $X$ em $\overline{\Sigma^{+}}$e se p é uma singularidade distinguida, então ela é definida como a órbita regular que possui $p \in \Sigma$ como ponto de chegada.

Definição 2.2.3. Uma separatriz que é simultaneamente estável e instável chamamos de conexão de separatrizes.

Vejamos nas próximas figuras como se comporta cada tipo de separatriz.

1. Separatriz instável de uma sela contida em $\Sigma^{+}$( veja a Figura 2.7).

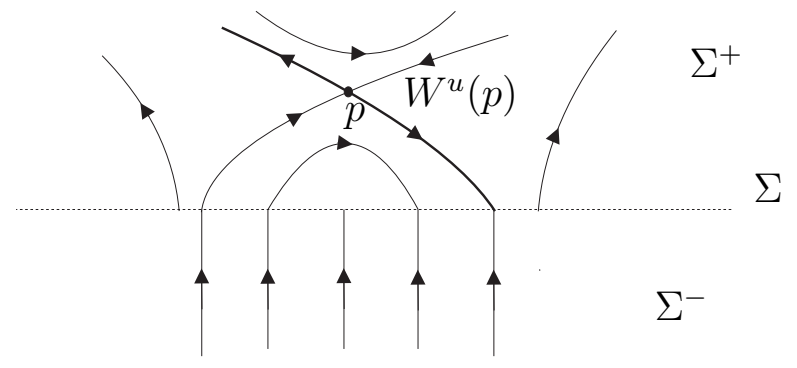

Figura 2.7: Separatriz instável.

2. Separatriz de uma singularidade distinguida (veja Figura 2.8).

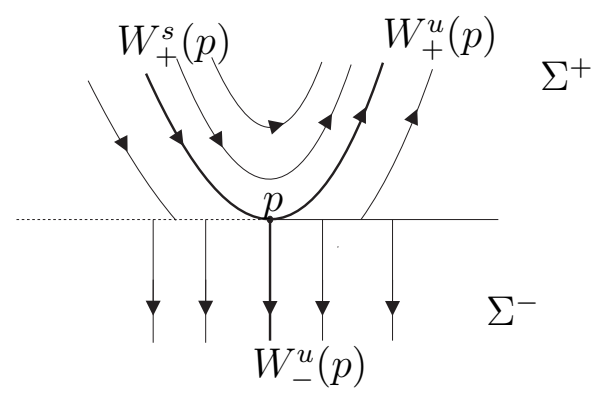

Figura 2.8: Separatriz de singularidade distinguida.

3. Conexão de separatrizes entre $p \in \Sigma^{+}$e $q \in \Sigma^{-}$(veja Figura 2.9).

Uma pergunta natural nesse estudo é a seguinte: O que são as órbitas periódicas em um sistema de Filippov? 


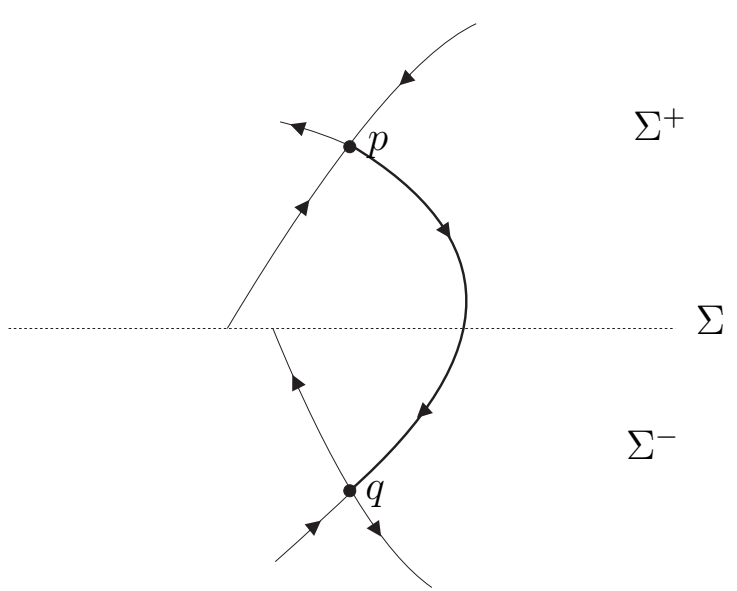

Figura 2.9: Conexão de separatrizes.

Nesse tipo de sistema, além das órbitas periódicas de $X$ em $\Sigma^{+}$e de $\mathrm{Y}$ em $\Sigma^{-}$, existem outras que não estão contidas em $\Sigma^{ \pm}$, mas apresentam comportamento semelhante. Vejamos como podemos definir esse tipo de órbita.

Definição 2.2.4. Uma órbita periódica regular é uma órbita regular

$$
\gamma=\left\{\varphi_{Z}(t, p) ; t \in \mathbb{R}\right\}
$$

que pertence a $\Sigma^{+} \cup \Sigma^{-} \cup \overline{\Sigma^{c}}$ e satisfaz

$$
\varphi_{Z}(t+T, p)=\varphi_{Z}(t, p)
$$

para algum $T>0$.

Uma representação geométrica é dada pela Figura 2.10.

Podemos também nos deparar com uma órbita periódica deslizante, que aparece quando $\Sigma$ é homeomorfa à $\mathbb{S}^{1}$ e $\Sigma=\Sigma^{s}$ ou $\Sigma=\Sigma^{e}$, de modo que o campo vetorial deslizante não possui pontos críticos.(Figura 2.11)

Observação 2.2.5. 1. No caso da órbita periódica deslizante, toda a subvariedade $\Sigma$ é uma órbita periódica. Como estamos interessados em estudar sistemas de Filippov localmente com $\Sigma$ uma curva localmente homeomorfa à $\mathbb{R}$ esse caso não será considerado. 


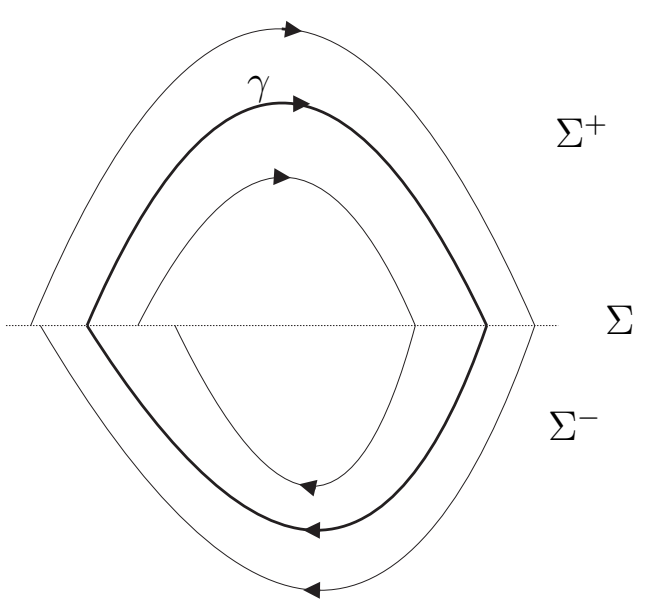

Figura 2.10: Órbita periódica

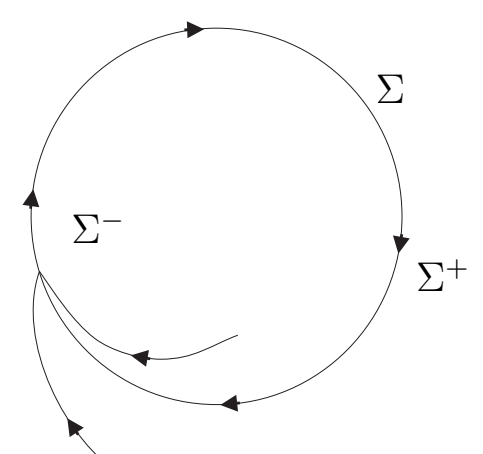

Figura 2.11: Órbita periódica deslizante 
2. Não existem órbitas periódicas que sejam combinações de movimentos regulares e deslizantes.

De fato, órbitas periódicas regulares só podem interceptar a curva $\Sigma$ em pontos de costura enquanto que em movimentos deslizantes, só temos região de deslize ou de escape.

A Observação 2.2.5 nos leva a pensar em como deve ser estudado um movimento periódico que envolva movimento deslizante e regular. Na Figura 2.12, temos um exemplo desse movimento, que caracteriza um ciclo, também conhecido como pseudo órbita.

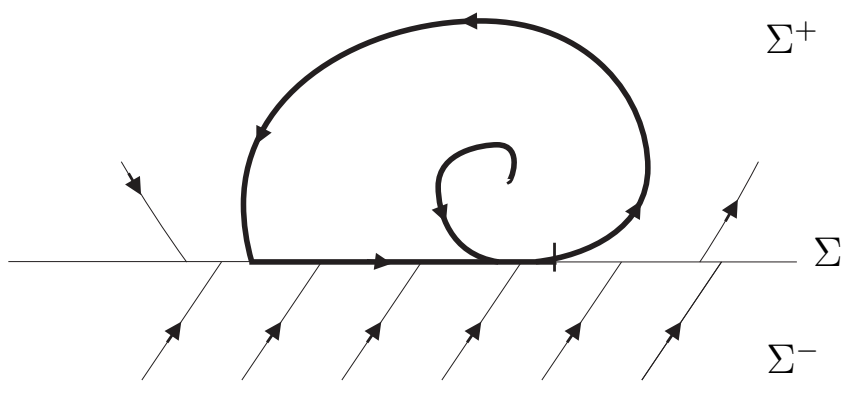

Figura 2.12: Ciclo ou pseudo órbita.

Definição 2.2.6. Um ciclo é o fecho de um conjunto finito de pedaços de órbitas $\gamma_{1}, \gamma_{2}, \ldots \gamma_{n}$, tal que $\gamma_{2 k}$ é um pedaço de órbita deslizante e $\gamma_{2 k+1}$ é uma órbita regular maximal, e os pontos de chegada e partida de $\gamma_{2 k+1}$ pertencem ao fecho das órbitas $\gamma_{2 k}$ e $\gamma_{2 k+2}$, respectivamente. Definimos o periodo do ciclo como sendo a soma dos tempos que é gasto em cada parte das trajetórias $\gamma_{i}$, com $i \in\{1,2, \ldots, n\}$.

As órbitas periódicas regulares também são chamadas órbitas periódicas padrão se elas estão em $\Sigma^{+}$ou $\Sigma^{-}$e órbitas periódicas de costura se elas interceptam $\overline{\Sigma^{c}}$.

Definição 2.2.7. Definimos um pseudociclo como o fecho de um conjunto de órbitas regulares $\gamma_{1}, \gamma_{2}, \ldots \gamma_{n}$ tal que uma das extremidades(os pontos de chegada e partida) de qualquer $\gamma_{i}$ coincide com uma extremidade de $\gamma_{i-1}$ e a outra, com uma extremidade de $\gamma_{i+1}$ (e também entre $\gamma_{1}$ e $\gamma_{n}$ ), formando uma curva homeomorfa a $\mathbb{S}^{1}$, de modo que em algum ponto, dois pontos de chegada ou partida coincidem.(Ver Figura 2.13) 


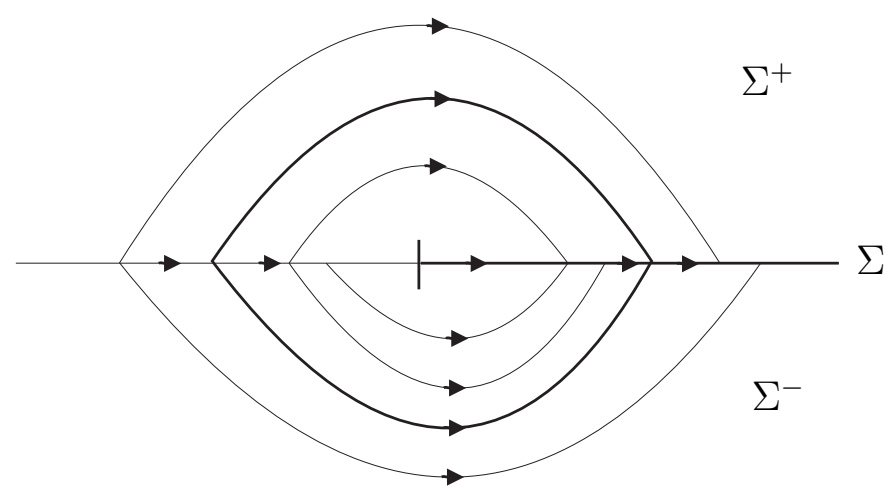

Figura 2.13: Pseudociclo

\subsection{Equivalência topológica}

Para finalizar a introdução aos sistemas de Filippov precisamos definir quando dois sistemas são equivalentes.

Definição 2.3.1. (E-equivalência) Dois sistemas de Filippov $Z$ e $\widetilde{Z}$ definidos em abertos $U$ e $\widetilde{U}$, com curvas de descontinuidade $\Sigma \subset U$ e $\widetilde{\Sigma} \subset \widetilde{U}$, respectivamente, são $\Sigma$ equivalentes se existe um homeomorfismo $h: U \rightarrow \widetilde{U}$ que preserva orientação e que leva órbitas de $Z$ em órbitas de $\widetilde{Z}$ e $\Sigma$ em $\widetilde{\Sigma}$.

Observação 2.3.2. 1. Se h é uma $\Sigma$-equivalência, então h envia órbitas regulares em órbitas regulares e singuralidades distinguidas em singularidades distinguidas.

De fato, como h é um homeomorfismo que leva órbitas em órbitas, então deve levar singularidades distinguidas em singularidades distinguidas. Ainda, como h preserva orientação, segue que leva órbitas regulares em órbitas regulares(as regiões de costura, deslize e escape são preservadas).

2. $h$ leva pontos de chegada em pontos de chegada e pontos de partida em pontos de partida.

3. Como as regiões de $\Sigma$ são preservadas, $h$ leva órbitas deslizantes em órbitas deslizantes, preserva separatrizes, conexões de separatrizes, órbitas periódicas, ciclos e pseudociclos. 


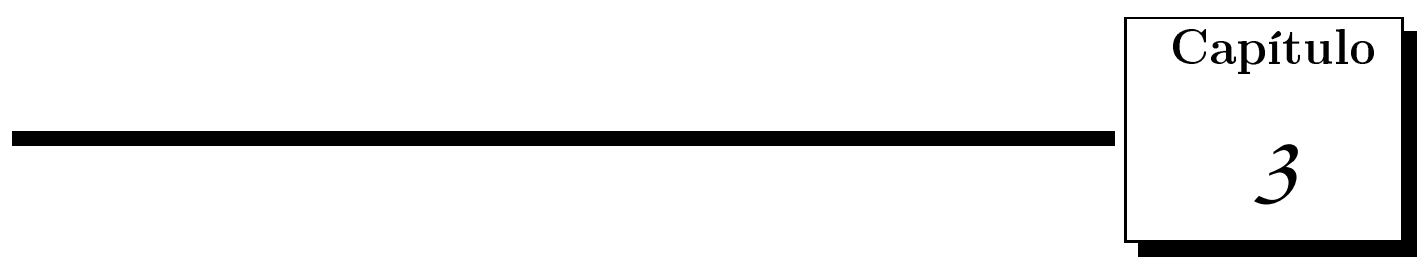

\section{$\mathrm{O}$ averaging para sistemas diferenciais}

\section{suaves por partes}

O objetivo deste capítulo é investigar a generalização da técnica do averaging para sistemas diferenciais suaves por partes. Após algumas definições, é possível estabelecer um resultado análogo ao Teorema da Existência e Unicidade, já conhecido do caso suave, para sistemas de Filippov. Ainda no contexto de generalizações, apresentamos o teorema do averaging para sistemas que sejam suaves por partes. Tal teorema nos ajuda a contar o número de soluções periódicas de um sistema do tipo $\dot{x}(t)=\varepsilon F(t, x)+\varepsilon^{2} R(t, x, \varepsilon)$ quando $F$ e $R$ são funções suaves por partes.

\subsection{Soluções de Filippov}

Para a investigação desejada, iniciaremos com uma breve introdução sobre os teoremas de existência e unicidade de soluções para sistemas com descontinuidades. Muitos dos resultados apresentados não serão demonstrados nesse trabalho, no entanto, quando esse for o caso, a referência é apresentada.

Neste trabalho, nosso foco principal está sobre os sistemas diferenciais descontínuos onde o conjunto de descontinuidade é uma variedade diferenciável, entretanto, dentro das equações diferenciais descontínuas existem sistemas mais gerais. Entendemos por sistema 
diferencial descontínuo um sistema do tipo

$$
\dot{x}(t)=X(t, x(t))
$$

onde a aplicação $X: \mathbb{R} \times \mathbb{R}^{n} \rightarrow \mathbb{R}^{n}$ é descontínua.

Em [12] encontramos uma definição bem intuitiva, dada por Caratheodory para o conceito de solução para esse tipo de sistema. Como a aplicação (3.1.1) pode ser multivalorada dependendo do ponto, a definição apresentada por Caratheodory não se aplica para muitos casos, uma vez que ela depende do valor do campo no ponto dado. Já Filippov [14] e Krasovskii [21] apresentaram definições mais abrangentes, que usaremos neste trabalho. Mais detalhes sobre as diferenças e semelhanças entre os conceitos de solução para sistemas do tipo (3.1.1) podem ser encontradas em [12].

Filippov trabalhou com o conceito de inclusão diferencial, que significa definir uma aplicação multivalorada $F(t, x(t))$ que associa a cada ponto um conjunto de possíveis soluções. Assim a cada sistema da forma (3.1.1) está associada a inclusão diferencial

$$
\dot{x}(t) \in F(t, x(t)) \text {. }
$$

A seguir apresentamos a definição da aplicação multivalorada de Filippov e algumas de suas propriedades.

Definição 3.1.1. Seja $X: \mathbb{R} \times \mathbb{R}^{n} \rightarrow \mathbb{R}^{n}$ campo vetorial, possivelmente descontínuo, associado ao sistema (3.1.1). A aplicação multivalorada de Filippov é a aplicação

$$
F[X]: \mathbb{R} \times \mathbb{R}^{n} \rightarrow \mathfrak{P}\left(\mathbb{R}^{n}\right)
$$

dada por

$$
F[X](t, x)=\bigcap_{\delta>0} \bigcap_{\mu(S)=0} \overline{c o}\{X(t, B(x, \delta) \backslash S)\}
$$

onde $\mathfrak{P}\left(\mathbb{R}^{n}\right)$ denota o conjunto das partes de $\mathbb{R}^{n}, \overline{c o}\{K\}$ o fecho convexo do conjunto $K$ e $\mu$ é a medida de Lebesgue.

Quando se trata de sistemas autônomos, a aplicação multivalorada possui algumas 
propriedades. Elas estão listadas abaixo e a prova das mesmas são dadas em [32].

Lema 3.1.2. (Consistência) Se $X: \mathbb{R}^{n} \rightarrow \mathbb{R}^{n}$ é contínuo em $x \in \mathbb{R}^{n}$ então

$$
F[X](x)=\{X(x)\}
$$

Lema 3.1.3. Se $X_{1}, X_{2}: \mathbb{R}^{n} \rightarrow \mathbb{R}^{n}$ são localmente limitados em $x \in \mathbb{R}^{n}$ então

$$
F\left[X_{1}+X_{2}\right](x) \subset F\left[X_{1}\right](x)+F\left[X_{2}\right](x),
$$

valendo a igualdade no caso dos campos serem contínuos.

Lema 3.1.4. (Regra do produto) $S e X_{1}, X_{2}: \mathbb{R}^{n} \rightarrow \mathbb{R}^{n}$ são localmente limitados em $x \in \mathbb{R}^{n}$ então

$$
F\left[\left\langle X_{1}, X_{2}\right\rangle\right](x) \subset F\left[X_{1}\right](x) \times F\left[X_{2}\right](x),
$$

valendo a igualdade no caso dos campos serem continuos.

Para sistemas não autônomos encontramos resultados análogos.

O próximo resultado nos diz que em casos específicos, a aplicação multivalorada de Filippov possui uma expressão simples.

Proposição 3.1.5. Se $X: \mathbb{R} \times \mathbb{R}^{n} \rightarrow \mathbb{R}^{n}$ é mensurável e localmente essencialmente limitado então, para cada $t \in \mathbb{R}$, existe $S_{t} \subset \mathbb{R}^{n}$ de medida nula tal que

$$
F[X](t, x)=\overline{c o}\left\{\lim _{i \rightarrow \infty} X\left(t, x_{i}\right): x_{i} \rightarrow x, x_{i} \neq S \cup S_{t}\right\}
$$

Com o objetivo de apresentar uma generalização do Teorema de existência para sistemas suaves por partes da forma (3.1.1) vamos definir soluções para inclusões diferenciais e condições para sua existência.

A distância entre dois conjuntos fechados $A, B \subset \mathbb{R}^{n}$ é definida como

$$
\alpha(A, B)=\max \{\beta(A, B), \beta(B, A)\},
$$


onde

$$
\beta(A, B)=\sup _{a \in A} d(a, B) \quad \text { e } \quad \beta(B, A)=\sup _{b \in B} d(A, b) .
$$

Definição 3.1.6. Seja $\Omega$ conjunto aberto e conexo. Dizemos que uma aplicação multivalorada $F: \Omega \rightarrow \mathfrak{P}(\Omega)$, onde $\mathfrak{P}(\Omega)$ é o conjunto das partes de $\Omega$, é contínua no ponto $p$ se $\alpha\left(F\left(p^{\prime}\right), F(p)\right) \rightarrow 0$ quando $p^{\prime} \rightarrow p$. Nas mesmas condições, é semi-contínua superiormente no ponto $p$, se $\beta\left(F\left(p^{\prime}\right), F(p)\right) \rightarrow 0$ quando $p^{\prime} \rightarrow p$.

Considere a inclusão diferencial

$$
\dot{x} \in F(t, x) \text {. }
$$

Definição 3.1.7. Uma solução da inclusão diferencial (3.1.2) é uma aplicação contínua $\gamma:\left[t_{0}, t_{1}\right] \rightarrow \mathbb{R}^{n}$ tal que $\dot{\gamma}(t)$ pertença ao conjunto $F(\gamma(t))$, para quase todo $t \in\left[t_{0}, t_{1}\right]$.

Definição 3.1.8. O domínio $\Omega$ e a aplicação multivalorada $F$ satisfazem as condições básicas se, $\forall x \in \Omega$ o conjunto $F(x)$ é não-vazio, limitado, fechado e convexo e a função $F$ é semi-contínua superiormente em $x$.

Apresentadas as definições acima estamos aptos para definir a solução de Filippov para um sistema descontínuo.

Utilizando a aplicação multivalorada de Filippov, associamos à cada equação diferencial da forma (3.1.1) a inclusão diferencial

$$
\dot{x}(t) \in F[X](t, x(t))
$$

Definição 3.1.9. Uma solução de Filippov de (3.1.1) é uma solução da inclusão (3.1.3).

Teorema 3.1.10. [14] Se $F: \Omega \rightarrow \mathfrak{P}(\Omega)$ e $\Omega$ satisfazem as condições básicas estabelecidas anteriormente, então dado $\left(t_{0}, x_{0}\right) \in \Omega$, existe uma solução da inclusão diferencial

$$
\dot{x} \in F(t, x), x\left(t_{0}\right)=x_{0} .
$$

Além disso, se o domínio $[0, T] \times D$ contém um cilindro $Z\left(t_{0} \leq t \leq t_{0}+a,\left|x-x_{0}\right| \leq b\right)$ 
então a solução existe no minimo para o intervalo

$$
t_{0} \leq t \leq t_{0}+d, d=\min \left\{a ; \frac{b}{m}\right\}, \operatorname{com} m=\sup _{Z}|F(t, x)|
$$

Observação 3.1.11. Note que com esse teorema podemos, sob algumas condições, extender as soluções de um sistema diferencial descontínuo, como discutido na Seção 1.5.2 para soluções de sistemas suaves.

\subsection{O grau de Brouwer sobre outro ponto de vista}

Na Seção 1.5.2 definimos o grau de Brouwer de uma função através do determinante da sua matriz Jacobiana e também apresentamos alguns exemplos. Essa forma de ver a função grau foi conveniente pois estávamos trabalhando com funções diferenciáveis e nesse caso, em geral, verificar que o determinante da matriz Jacobiana é não nulo é simples. Entretanto, agora estamos trabalhando com sistemas suaves por partes e gostaríamos de estudar o conceito de grau de Brouwer para a classe das funções contínuas. A seguir, dois teoremas serão fundamentais para essa generalização e a prova deles está feita em [10].

Teorema 3.2.1. Sejam $X=Y=\mathbb{R}^{n}$ onde $n$ é inteiro positivo. Tome $\Omega$ subconjunto aberto e limitado de $X$ e considere a aplicação continua $f: \bar{\Omega} \rightarrow Y$ e $y_{0} \in Y$ tais que $y_{0} \notin f(\partial \Omega)$. Então, para cada tripla $\left(f, \Omega, y_{0}\right)$ corresponde um inteiro $d\left(f, \Omega, y_{0}\right)$ satisfazendo as seguintes propriedades:

(i) Se $d\left(f, \Omega, y_{0}\right) \neq 0$ então $y_{0} \in f(\Omega)$. Se fo for a aplicação identidade de $X$ em $Y$ então, para cada conjunto aberto e limitado $\Omega$ e $y_{0} \in \Omega$, temos

$$
d\left(f_{0}, \Omega, y_{0}\right)=+1
$$

(ii) (Aditividade) Se $f: \bar{\Omega} \rightarrow Y$ é uma aplicação contínua onde $\Omega=\Omega_{1} \cup \Omega_{2}$ é subconjunto aberto e limitado de $X$ e $\Omega_{1}$ e $\Omega_{2}$ formam um par de subconjuntos abertos disjuntos de $\Omega$ tais que

$$
y_{0} \notin f\left(\partial \Omega_{1}\right) \cup f\left(\partial \Omega_{2}\right),
$$


então

$$
d\left(f, \Omega, y_{0}\right)=d\left(f_{\Omega_{1}}, \Omega_{1}, y_{0}\right)+d\left(f_{\Omega_{2}}, \Omega_{2}, y_{0}\right)
$$

(iii) (Invariância por homotopia) Seja $\Omega$ um subconjunto aberto e limitado de $X$ e considere uma homotopia contínua $\left\{f_{t} ; 0 \leq t \leq 1\right\}$ de aplicações de $\bar{\Omega}$ em $Y$. Seja $\left\{y_{t} ; 0 \leq t \leq 1\right\}$ uma curva continua em $Y$ tal que $y_{t} \notin f_{t}(\partial \Omega)$, para qualquer $t \in[0,1]$. Então, $d\left(f_{t}, \Omega, y_{t}\right)$ é constante para $t \in[0,1]$.

Teorema 3.2.2. A função grau $d\left(f, \Omega, y_{0}\right)$, chamada grau de Brouwer da função $f$, é unicamente determinada pelas três condições apresentadas no Teorema 3.2.1. Denotamosa por $d_{B}\left(f, G, y_{0}\right)$.

Note que a Definição 1.5.3 satisfaz as três hipóteses do Teorema 3.2.1 logo pelo Teorema 3.2.2 as funções coincidem no caso em que $f$ é $C^{1}$. Portanto o grau de Brouwer definido acima satisfaz as propriedades descritas na seção 1.5.2.

\subsection{A técnica do averaging}

Com o objetivo de generalizar a teoria do averaging para sistemas suaves por partes, será útil introduzir o processo de regularização. Esse processo permite aproximar cada função contínua por partes $Z(t, x)$ por uma família a um parâmetro de funções contínuas $Z_{\delta}(t, x)$. Em [37], Sotomayor e Teixeira introduziram essa técnica para sistemas suaves por partes em $\mathbb{R}^{2}$ com uma linha de descontinuidade.

Para a realização do processo precisamos de uma função de transição, indicada por $\phi$.

Definição 3.3.1. Uma função $\phi: \mathbb{R} \rightarrow \mathbb{R}$ de classe $C^{r}$ é uma função de transição se $\phi(u)=-1$ para $u \leq-1, \phi(u)=1$ para $u \geq 1$ e $\phi^{\prime}(u)>0$ se $u \in(-1,1)$.

Definição 3.3.2. A $\phi$-regularização de classe $C^{r}$ do campo $Z=(X, Y)$ é a família a um parâmetro de funções contínuas $Z_{\delta}$ dada por

$$
Z_{\delta}(t, x)=\frac{1}{2}(X(t, x)+Y(t, x))+\frac{1}{2} \phi_{\delta}(h(t, x))(X(t, x)-Y(t, x)),
$$

$\operatorname{com} \phi_{\delta}(u)=\phi\left(\frac{u}{\delta}\right)$ 
Proposição 3.3.3.

$$
\lim _{\delta \rightarrow 0} Z_{\delta}(t, x)=Z(t, x)
$$

para todo $(t, x) \in(\mathbb{R} \times D) \backslash \Sigma$.

Demonstração: Consideremos a função sign definida em $\mathbb{R} \backslash\{0\}$ por

$$
\operatorname{sign}(x)= \begin{cases}1 & \text { se } x>0 \\ -1 & \text { se } x<0\end{cases}
$$

O sistema (2.0.1) é escrito como

$$
x^{\prime}(t)=Z(t, x)=F_{1}(t, x)+\operatorname{sign}(h(t, x)) F_{2}(t, x),
$$

onde $F_{1}(t, x)=\frac{1}{2}(X(t, x)+Y(t, x))$ e $F_{2}(t, x)=\frac{1}{2}(X(t, x)-Y(t, x))$.

Para a $\phi$-regularização do sistema (3.3.1) definimos a função de transição

$$
\phi(u)= \begin{cases}1 & \text { se } u \geq 1 \\ u & \text { se } u \in(-1,1) \\ -1 & \text { se } u \leq-1 .\end{cases}
$$

e $\phi_{\delta}: \mathbb{R} \rightarrow \mathbb{R}$ a função contínua definida por $\phi_{\delta}(u)=\phi\left(\frac{u}{\delta}\right)$. Se $u \neq 0$ e $\delta \rightarrow 0$, então $\frac{u}{\delta} \rightarrow \pm \infty$ (dependendo de $u$ ser positivo ou negativo). Logo,

$$
\lim _{\delta \rightarrow 0} \phi_{\delta}(u)=\operatorname{sign}(u), \quad \forall u \neq 0
$$

e então

$$
Z_{\delta}(t, x)=F_{1}(t, x)+\phi_{\delta}(h(t, x)) F_{2}(t, x)
$$

é uma $\phi$ - regularização de classe $C^{0}$ do sistema (3.3.1).

A ideia da $\phi$-regularização de um sistema suave por partes por uma família de sistemas suaves é a chave para generalização da teoria do averaging para sistemas de Filippov. Llibre-Novaes-Teixeira em [28] regularizaram o sistema descontínuo $Z$, aplicaram a teoria do averaging para o sistema regularizado e contínuo $Z_{\delta}$ tal que $Z_{\delta} \rightarrow Z$ quando $\delta \rightarrow 0$ 
e demonstraram a validade do resultado para o sistema $Z$. Vejamos o que diz o teorema principal.

Teorema 3.3.4. Considere o seguinte sistema diferencial:

$$
\dot{x}(t)=\varepsilon F(x, t)+\varepsilon^{2} R(t, x, \varepsilon),
$$

onde $F(t, x)=F_{1}(t, x)+\operatorname{sign}(h(t, x)) F_{2}(t, x), R(t, x)=R_{1}(t, x)+\operatorname{sign}(h(t, x)) R_{2}(t, x) e$ $F_{1}, F_{2}: \mathbb{R} \times D \rightarrow \mathbb{R}^{n}, R_{1}, R_{2}: \mathbb{R} \times D \times\left(-\varepsilon_{0}, \varepsilon_{0}\right) \rightarrow \mathbb{R}^{n}$ e $h: \mathbb{R} \times D \rightarrow \mathbb{R}$ são funções contínuas, T-periódicas na variável $t$ e $D \subset \mathbb{R}^{n}$ é aberto. Suponha também que $h$ seja uma função de classe $C^{1}$ tal que $0 \in \mathbb{R}$ seja um valor regular.

Defina a função $f: D \rightarrow \mathbb{R}^{n}$ como sendo

$$
f(x)=\int_{0}^{T} F(t, x) d t
$$

que daqui em diante chamaremos de função promediada e considere válidas as seguintes hipóteses:

i) $F_{1}, F_{2}, R_{1}, R_{2}$ e h são localmente Lipschitz com respeito à variável $x$;

ii) existe um aberto limitado $C \subset D$ tal que, para $|\varepsilon|>0$ suficientemente pequeno, toda órbita começando em $C$ atinge a região de descontinuidade somente em pontos da região de costura;

iii) para $a \in C, \operatorname{com} f(a)=0$, existe uma vizinhança $U \subset C$ de a tal que $f(z) \neq 0$, para todo $z \in \bar{U} \backslash\{a\}$ e $d_{B}(f, U, 0) \neq 0$.

Então, para $|\varepsilon|>0$ suficientemente pequeno, existe uma solução $x(t, \varepsilon)$ de (3.3.2) que é T-periódica e tal que $x(0, \varepsilon) \rightarrow$ a se $\varepsilon \rightarrow 0$.

É possível exigir menos das hipóteses do Teorema 3.3.4, como discutimos a seguir. Considere o seguinte conjunto

$$
D_{0}=\left\{z \in D ; h_{z}: t \in[0, T] \mapsto h(t, z) \text { possui somente zeros isolados }\right\}
$$


Como $h$ é de classe $C^{1}$ segue que int $D_{0} \neq \emptyset$. Nesse caso temos

Teorema 3.3.5. Assumindo válidas as hipóteses i) e iii) do Teorema 3.3.4 e a seguinte condição

ii') existe um aberto limitado $C \subset D_{0}$ tal que, para $\varepsilon>0$ suficientemente pequeno, toda órbita começando em $C$ atinge a variedade de descontinuidade somente em pontos da região de costura.

Então para $\varepsilon>0$ suficientemente pequeno, existe uma solução $T$-periódica $x(t, \varepsilon)$ do sistema (3.3.2) satisfazendo $x(0, \varepsilon) \rightarrow$ a quando $\varepsilon \rightarrow 0$.

Observação 3.3.6. A principal diferença entre os Teoremas 3.3.4 e 3.3 .5 é que no segundo resultado exigimos que a hipótese ii) valha para $\varepsilon>0$.

Os próximos resultados fornecem condições sob as quais a hipótese ii) do Teorema 3.3.4 e $i i^{\prime}$ ) do Teorema 3.3.5 são válidas.

Proposição 3.3.7. Suponhamos que $\partial_{t} h(t, z) \neq 0$ para todo $(t, z) \in \Sigma$. Então, para $z \in D$, a aplicação $h_{z}: t \mapsto h(t, z)$, para $t \in[0, T]$ se anula somente em um conjunto finito de pontos.

Demonstração: Por hipótese, a aplicação $h_{z}: t \mapsto h(t, z)$ é uma função de classe $C^{1}$. Suponha por absurdo que exista uma sequência estritamente crescente $\left(t_{i}\right)_{i \in \mathbb{N}} \subset[0, T]$ tal que $h_{z}\left(t_{i}\right)=0$ para todo $i \in \mathbb{N}$. Sendo $[0, T]$ compacto, existe uma subsequência convergente $\left(t_{i_{j}}\right)_{j \in \mathbb{N}}$ tal que $t_{i_{j}} \rightarrow \bar{t} \in[0, T]$ quando $j \rightarrow \infty$. Dado que $h_{z}$ é contínua, segue que $h_{z}(\bar{t})=0$ e então $(\bar{t}, z) \in \Sigma$. Aplicando o Teorema do Valor Médio para cada $j \in \mathbb{N}$, garantimos que existe $s_{j} \in\left(t_{i_{j}}, t_{i_{j+1}}\right)$ tal que $h_{z}^{\prime}\left(s_{j}\right)=0$. Considerando a sequência $\left(s_{j}\right)_{j \in \mathbb{N}}$, temos que $s_{j} \rightarrow \bar{t}$ e então $h_{z}^{\prime}(\bar{t})=0$, contrariando a hipótese.

Proposição 3.3.8. Suponha que $\partial_{t} h(t, x) \neq 0$, para cada $(t, x) \in \Sigma=h^{-1}(0)$. Então a hipótese ii) é válida.

Demonstração: Escreva (3.3.2) como um sistema autônomo da seguinte forma

$$
\left(\begin{array}{c}
\tau^{\prime} \\
x^{\prime}
\end{array}\right)= \begin{cases}X(\tau, x) & \text { se } h(\tau, x)>0 \\
Y(\tau, x) & \text { se } h(\tau, x)<0\end{cases}
$$


onde

$$
\begin{gathered}
X(\tau, x)=\left(\begin{array}{c}
1 \\
\varepsilon\left(F_{1}(\tau, x)+F_{2}(\tau, x)\right)+\varepsilon^{2}\left(R_{1}(\tau, x, \varepsilon)+R_{2}(\tau, x, \varepsilon)\right)
\end{array}\right) \\
Y(\tau, x)=\left(\begin{array}{c}
1 \\
\varepsilon\left(F_{1}(\tau, x)-F_{2}(\tau, x)\right)+\varepsilon^{2}\left(R_{1}(\tau, x, \varepsilon)-R_{2}(\tau, x, \varepsilon)\right)
\end{array}\right) .
\end{gathered}
$$

Assim sendo, temos

$$
\begin{aligned}
(X h)(Y h)= & \langle\nabla h, X\rangle\langle\nabla h, Y\rangle \\
= & \left(\partial_{t} h\right)^{2}+2 \varepsilon \partial_{t} h\left\langle\nabla_{x} h, F_{1}\right\rangle+\varepsilon^{2}\left(2 \partial_{t} h\left\langle\nabla_{x} h, R_{1}\right\rangle+\left\langle\nabla_{x} h, F_{1}\right\rangle^{2}-\right. \\
& \left.\left\langle\nabla_{x} h, F_{2}\right\rangle^{2}\right)+2 \varepsilon^{3}\left(\left\langle\nabla_{x} h, F_{1}\right\rangle\left\langle\nabla_{x} h, R_{1}\right\rangle-\left\langle\nabla_{x} h, F_{2}\right\rangle\left\langle\nabla_{x} h, R_{2}\right\rangle\right)+ \\
& \varepsilon^{4}\left(\left\langle\nabla_{x} h, R_{1}\right\rangle^{2}-\left\langle\nabla_{x} h, R_{2}\right\rangle^{2}\right) .
\end{aligned}
$$

Denote por $x(t, z)$ a solução de (3.3.2) satisfazendo $x(0, z)=z$. Pela Proposição 3.3.7 segue que $D_{0} \neq \emptyset$, assim, se $U \subset D_{0}$ é aberto e limitado defina $K=\{(t, x(t, z)) ;(t, z) \in$ $[0, T] \times \bar{U}\} \subset[0, T] \times D$. Observe que $K$ é compacto pois $[0, T]$ é compacto e a solução $x=x(t, z)$ é uma função contínua.

Sendo $\left(\partial_{t} h(t, x)\right)^{2}$ uma função positiva em $\mathbb{R} \times D$, existe $k_{0}>0$ tal que $\left(\partial_{t} h(t, x)\right)^{2}>k_{0}$ para todo $(t, x) \in K$. Sendo assim, podemos escolher $\left|\varepsilon_{0}\right|>0$ tal que $(X h)(Y h)(t, x)>0$ para todo $(t, x) \in K \cap \Sigma \mathrm{e} \varepsilon \in\left(-\varepsilon_{0}, \varepsilon_{0}\right)$.

A Proposição 3.3.8 também fornece uma condição suficiente para que $i i^{\prime}$ ) se verifique. Se $\partial_{t} h(p)=0$ para algum $p \in \Sigma$, temos o seguinte resultado.

Proposição 3.3.9. Suponha que para cada $p \in \Sigma$ tal que $\partial_{t} h(p)=0$, exista uma função continua positiva $\xi_{p}: \mathbb{R} \times D \rightarrow \mathbb{R}$ para a qual a desigualdade

$$
\left(\partial_{t} h\left\langle\partial_{x} h, F_{1}\right\rangle+\varepsilon \frac{\left\langle\partial_{x} h, F_{1}\right\rangle^{2}-\left\langle\partial_{x} h, F_{2}\right\rangle^{2}}{2}\right)(t, x) \geq \frac{\xi_{p}(t, x)}{2}
$$

se verifique para $(t, x) \in \Sigma_{p}$, onde $\Sigma_{p}$ é a componente conexa de $\Sigma$ que contém $p$. Então a condição $\left.i i^{\prime}\right)$ é válida. 
Demonstração: De (3.3.4), segue que:

$(X h)(Y h)=\left(\partial_{t} h+\varepsilon^{2}\left\langle\nabla_{x} h, R_{1}\right\rangle\right)^{2}+2 \varepsilon\left(\partial_{t} h\left\langle\partial_{x} h, F_{1}\right\rangle+\varepsilon \frac{\left\langle\partial_{x} h, F_{1}\right\rangle^{2}-\left\langle\partial_{x} h, F_{2}\right\rangle^{2}}{2}\right)+\mathcal{O}\left(\varepsilon^{3}\right)$.

Usando a notação da Proposição 3.3.8, afirmamos que $K \cap \Sigma$ possui um número finito de componentes conexas. De fato, como $\Sigma$ é uma variedade diferenciável em $[0, T] \times D$, segue que ela pode ser coberta com bolas abertas, através de homeomorfismos. Como $K \cap \Sigma \subset K$, segue que $K \cap \Sigma$ é compacto e portanto admite subcobertura finita por bolas abertas e conexas, donde segue a afirmação.

Dessa maneira podemos escolher um subconjunto finito $\left\{p_{1}, p_{2}, \ldots, p_{m}\right\} \subset \Sigma$ de tal forma que $\partial_{t} h\left(p_{i}\right)=0$ para $i=1,2, \ldots, m$, com $\Sigma_{p_{i}} \cap \Sigma_{p_{j}}=\emptyset$ se $i \neq j$ e $\partial_{t} h(t, x) \neq 0$ para todo $(t, x) \in \Sigma \backslash\left(\Sigma_{p_{1}} \cup \Sigma_{p_{2}} \cup \ldots \cup \Sigma_{p_{m}}\right)$. Assim temos cada zero de $\partial h_{t}$ em uma das componentes conexas de $\Sigma$.

Da hipótese segue que

$$
\begin{aligned}
(X h)(Y h)(t, x) & \geq \varepsilon \xi_{p_{i}}(t, x)+\mathcal{O}\left(\varepsilon^{2}\right)+\frac{\left(\partial_{t} h+\varepsilon^{2}\left\langle\partial_{x} h, R_{1}\right\rangle\right)^{2}}{2 \varepsilon} \\
& \geq \varepsilon \xi_{p_{i}}(t, x)+\mathcal{O}\left(\varepsilon^{3}\right)
\end{aligned}
$$

para $i=1,2, \ldots m$ e $(t, x) \in \Sigma_{p_{i}}$.

Como $\xi_{p_{i}}: \mathbb{R} \times D \rightarrow \mathbb{R}$ é uma função contínua positiva e $K$ é compacto, existe $k_{i}>0$ tal que $\xi_{p_{i}}(t, x)>k_{i}$ para todo $(t, x) \in K$. Então, escolhemos $\varepsilon_{i}$ suficientemente pequeno de tal forma que $(X h)(Y h)(t, x)>0$ para todo $(t, x) \in K \cap \Sigma_{p_{i}}$ e $\varepsilon \in\left(0, \varepsilon_{i}\right)$. Por outro lado, pela Proposição 3.3.8, podemos escolher $\varepsilon_{0}>0$ suficientemente pequeno tal que $(X h)(Y h)(t, x)>0$ para todo $(t, x) \in K \cap \Sigma \backslash\left(\bigcup_{i=1}^{m} \Sigma_{p_{i}}\right)$ e $\varepsilon \in\left(-\varepsilon_{0}, \varepsilon_{0}\right)$. Escolhendo $\bar{\varepsilon}=\min \left\{\varepsilon_{0}, \varepsilon_{1}, \ldots, \varepsilon_{m}\right\}$, concluímos que $(X h)(Y h)(t, x)>0$ para todo $(t, x) \in K \cap \Sigma \mathrm{e}$ $\varepsilon \in(0, \bar{\varepsilon})$.

Para a demonstração dos Teorema 3.3.4 e 3.3.5, precisaremos de alguns resultados auxiliares. Note que em ambas as versões, trabalhamos com o grau de Brouwer da função promediada $f$ e portanto precisamos provar primeiramente que esta função é contínua para que a função grau esteja bem definida. No que segue abaixo, $\mu$ representa a medida de Lebesgue. 
Lema 3.3.10. A função promediada $f(x)=\int_{0}^{T} F(t, x) d t$ é contínua em $C$.

Demonstração: Tanto a hipótese $i i^{\prime}$ ) do Teorema 3.3.5 quanto a hipótese $i i$ ) do Teorema 3.3.4 nos garantem que em $C$ a aplicação $h_{z}: t \mapsto h(t, z)$ possui somente zeros isolados. Esse fato é imediato para o Teorema 3.3.5 dado que $C \subset D_{0}$, mas não é tão simples para o Teorema 3.3.4. Ele é uma consequência da Afirmação 3.4.1.

Para cada $z \in C$ defina os conjuntos

$$
\begin{aligned}
& A_{z}^{+}=\{t \in[0, T]: h(t, z)>0\}, \\
& A_{z}^{-}=\{t \in[0, T]: h(t, z)<0\}, \\
& A_{z}^{0}=\{t \in[0, T]: h(t, z)=0\} .
\end{aligned}
$$

Como os zeros de $h(t, z)$ são isolados para $z \in C$, segue que $\mu\left(A_{z}{ }^{0}\right)=0$. Além disso, podemos escrever $[0, T]=\overline{A_{z}{ }^{+} \cup A_{z}{ }^{-}}$.

Fixe $z_{0} \in C$. Para cada $z \in C$ numa vizinhança de $z_{0}$ temos

$$
\begin{aligned}
\mid f(z)- & f\left(z_{0}\right)|=| \int_{0}^{T} F_{1}\left(t, z_{0}\right)+\operatorname{sign}\left(h\left(t, z_{0}\right)\right) F_{2}\left(t, z_{0}\right)-F_{1}(t, z)-\operatorname{sign}(h(t, z)) F_{2}(t, z) d t \mid \\
& \leq \int_{0}^{T}\left|F_{1}\left(t, z_{0}\right)-F_{1}(t, z)\right| d t+\int_{0}^{T}\left|\operatorname{sign}\left(h\left(t, z_{0}\right)\right) F_{2}\left(t, z_{0}\right)-\operatorname{sign}(h(t, z)) F_{2}(t, z)\right| d t \\
& \leq L T\left|z-z_{0}\right|+\int_{A_{z_{0}}^{+} \cap A_{z}^{+}}\left|F_{2}\left(t, z_{0}\right)-F_{2}(t, z)\right| d t+\int_{A_{z_{0}}^{-} \cap A_{z}^{-}}\left|F_{2}\left(t, z_{0}\right)-F_{2}(t, z)\right| d t \\
& +\int_{A_{z_{0}}^{-} \cap A_{z}^{+}}\left|F_{2}\left(t, z_{0}\right)+F_{2}(t, z)\right| d t+\int_{A_{z_{0}}^{+} \cap A_{z}^{-}}\left|F_{2}\left(t, z_{0}\right)+F_{2}(t, z)\right| d t \\
& \leq 3 T L\left|z-z_{0}\right|+M\left(\mu\left(A_{z_{0}}^{+} \cap A_{z}^{-}\right)+\mu\left(A_{z_{0}}^{-} \cap A_{z}^{+}\right)\right),
\end{aligned}
$$

onde $L$ é a constante de Lipschitz de $F_{1}$ e $M=\max \left\{F_{2}(t, z):(t, z) \in[0, t] \times \bar{U}\right\}<\infty$.

Observe que quando $z \rightarrow z_{0}, \mu\left(A_{z_{0}}^{+} \cap A_{z}^{-}\right)$e $\mu\left(A_{z_{0}}^{-} \cap A_{z}^{+}\right)$tendem a zero, pois $h$ é uma função de classe $C^{1}$ e isso finaliza a demonstração do lema.

Como vimos no Capítulo 2, temos

$$
\Sigma^{+}=\{(t, x) \in \mathbb{R} \times D: h(t, x)>0\}, \quad \Sigma^{-}=\{(t, x) \in \mathbb{R} \times D: h(t, x)<0\}
$$

e $R \times D=\Sigma^{+} \cup \Sigma \cup \Sigma^{-}$. A próxima proposição, demonstrada em [14], garante que as 
soluções de Filippov pelos pontos de $C$ estão bem definidas.

Proposição 3.3.11. Para todo ponto da variedade $\Sigma$ onde $(X h)(Y h)>0$, existe uma única solução passando de $\Sigma^{-} \grave{a} \Sigma^{+}$ou vice-versa.

Lema 3.3.12. Para $|\varepsilon|>0$ (ou $\varepsilon>0)$ suficientemente pequeno, as soluções de Filippov do sistema (3.3.2) começando em C estão unicamente definidas.

Demonstração: Da hipótese $i i)$, segue que as soluções que partem de $C$ só cruzam a variedade de descontinuidade em pontos da região de costura. Aplicando a Proposição 3.3.11 concluímos o resultado.

Como mencionamos anteriormente, a ideia é trabalhar com um sistema contínuo que aproxime (de certa forma) o sistema descontínuo a ser estudado. Para isso, defina as seguintes funções contínuas

$$
\begin{gathered}
F_{\delta}(t, x)=F_{1}(t, x)+\phi_{\delta}(h(t, x)) F_{2}(t, x), \\
R_{\delta}(t, x, \varepsilon)=R_{1}(t, x, \varepsilon)+\phi_{\delta}(h(t, x)) R_{2}(t, x, \varepsilon),
\end{gathered}
$$

onde $\phi_{\delta}(u)=\phi\left(\frac{u}{\delta}\right)$ e $\phi: \mathbb{R} \rightarrow \mathbb{R}$ é dada por

$$
\phi(u)= \begin{cases}1 & \text { se } u \geq 1 \\ u & \text { se } u \in(-1,1) \\ -1 & \text { se } u \leq-1 .\end{cases}
$$

Note que $F_{\delta} \rightarrow F, R_{\delta} \rightarrow R$ e $\phi_{\delta}(u) \rightarrow \operatorname{sign}(u)$ quando $\delta \rightarrow 0$.

Considere agora o seguinte sistema contínuo

$$
x^{\prime}(t)=\varepsilon F_{\delta}(t, x)+\varepsilon^{2} R_{\delta}(t, x, \varepsilon) .
$$

Para esse sistema, a função promediada é dada por

$$
f_{\delta}(z)=\int_{0}^{T} F_{\delta}(t, x) d t .
$$


Queremos aplicar o Teorema do averaging 1.5.15 para o sistema (3.3.5) e para isso precisamos verificar as hipóteses desse teorema. Isso é feito nos próximos lemas.

Lema 3.3.13. $F_{\delta}$ e $R_{\delta}$ são funções T-periódicas.

Demonstração: De fato, temos

$$
\begin{aligned}
F_{\delta}(t+T, x) & =F_{1}(t+T, x)+\phi_{\delta}(h(t+T, x)) F_{2}(t+T, x) \\
& =F_{1}(t, x)+\phi_{\delta}(h(t, x)) F_{2}(t, x)
\end{aligned}
$$

uma vez que $F_{1}, F_{2}$ e $h$ são funções $T$-periódicas em $t$.

A prova para $R_{\delta}$ é análoga, substituindo $F_{1}$ e $F_{2}$ por $R_{1}$ e $R_{2}$, respectivamente.

Lema 3.3.14. Para $\delta \in(0,1]$ a função $\phi_{\delta}: \mathbb{R} \rightarrow \mathbb{R}$ é globalmente $\frac{1}{\delta}$-Lipschitz.

Demonstração: Vamos dividir a prova em casos. Sejam $u_{1}, u_{2} \in \mathbb{R}$

- Se $u_{1}, u_{2} \geq \delta$ ou $u_{1}, u_{2} \leq-\delta$ :

$$
\left|\phi_{\delta}\left(u_{1}\right)-\phi_{\delta}\left(u_{2}\right)\right|=0 \leq \frac{1}{\delta}\left|u_{1}-u_{2}\right| .
$$

- Se $u_{1}, u_{2} \notin(-\delta, \delta)$ e não se encaixam no caso anterior

$$
\left|\phi_{\delta}\left(u_{1}\right)-\phi_{\delta}\left(u_{2}\right)\right|=2<\frac{1}{\delta}\left|u_{1}-u_{2}\right| .
$$

- Se $u_{1}, u_{2} \in(-\delta, \delta)$

$$
\left|\phi_{\delta}\left(u_{1}\right)-\phi_{\delta}\left(u_{2}\right)\right|=\left|\frac{u_{1}}{\delta}-\frac{u_{2}}{\delta}\right|=\frac{1}{\delta}\left|u_{1}-u_{2}\right| .
$$

- Se $u_{1} \in(-\delta, \delta)$ e $u_{2} \notin(-\delta, \delta)$

Suponha, sem perda de generalidade, que $u_{2} \geq \delta$

$$
\left|\phi_{\delta}\left(u_{1}\right)-\phi_{\delta}\left(u_{2}\right)\right|=\left|\frac{u_{1}}{\delta}-1\right|=1-\frac{u_{1}}{\delta} \leq \frac{u_{2}}{\delta}-\frac{u_{1}}{\delta}=\frac{u_{2}-u_{1}}{\delta} .
$$


Proposição 3.3.15. Para $\delta \in(0,1]$ as funções $F_{\delta}$ e $R_{\delta}$ são localmente Lipschitz com respeito à variável $x$.

Demonstração: Seja $K \subset D$ um subconjunto compacto e $M=\sup \left\{\left|F_{2}(t, x)\right| ;(t, x) \in\right.$ $[0, T] \times K\}$. Para $x_{1}, x_{2} \in K$, onde $F_{1}$ e $h$ são localmente Lipschitz, temos

$$
\begin{aligned}
\left|F_{\delta}\left(t, x_{1}\right)-F_{\delta}\left(t, x_{2}\right)\right| & =\left|F_{1}\left(t, x_{1}\right)-F_{1}\left(t, x_{2}\right)+\phi_{\delta}\left(h\left(t, x_{1}\right)\right) F_{2}\left(t, x_{1}\right)-\phi_{\delta}\left(h\left(t, x_{2}\right)\right) F_{2}\left(t, x_{2}\right)\right| \\
& \leq L\left|x_{1}-x_{2}\right|+\left|\phi_{\delta}\left(h\left(t, x_{1}\right)\right) F_{2}\left(t, x_{1}\right)-\phi_{\delta}\left(h\left(t, x_{1}\right)\right) F_{2}\left(t, x_{2}\right)\right| \\
& +\left|\phi_{\delta}\left(h\left(t, x_{1}\right)\right) F_{2}\left(t, x_{2}\right)-\phi_{\delta}\left(h\left(t, x_{2}\right)\right) F_{2}\left(t, x_{2}\right)\right| \\
& \leq L\left|x_{1}-x_{2}\right|+\left|\phi_{\delta}\left(h\left(t, x_{1}\right)\right)\right|\left|F_{2}\left(t, x_{1}\right)-F_{2}\left(t, x_{2}\right)\right| \\
& +|| F_{2}\left(t, x_{2}\right)||\left|\phi_{\delta}\left(h\left(t, x_{1}\right)\right)-\phi_{\delta}\left(h\left(t, x_{2}\right)\right)\right| \\
& \leq L\left|x_{1}-x_{2}\right|+L\left|x_{1}-x_{2}\right|+\frac{M}{\delta}\left|h\left(t, x_{1}\right)-h\left(t, x_{2}\right)\right| \\
& \leq 2 L\left|x_{1}-x_{2}\right|+\frac{M L}{\delta}\left|x_{1}-x_{2}\right|=L_{\delta}\left|x_{1}-x_{2}\right|,
\end{aligned}
$$

$\operatorname{com} L_{\delta}=\left(2 L+\frac{M L}{\delta}\right)$ e $L$ é o máximo entre as constantes de Lipschitz de $F_{1}$ e $F_{2}$.

A demonstração para $R_{\delta}$ é análoga.

Na próxima seção apresentamos a prova do Teorema 3.3.4. A prova do Teorema 3.3.5 é inteiramente análoga, apenas substituindo $\varepsilon>0$ por $|\varepsilon|>0$, portanto será omitida nessa dissertação.

\subsection{Demonstração do Teorema 3.3.4}

Considere a aplicação de Poincaré para os sistemas

$$
\dot{x}(t)=\varepsilon F(t, x)+\varepsilon^{2} R(t, x, \varepsilon),
$$

e

$$
\dot{x}_{\delta}(t)=\varepsilon F_{\delta}(t, x)+\varepsilon^{2} R_{\delta}(t, x, \varepsilon),
$$

onde $F_{\delta}(t, x)=F_{1}(t, x)+\phi_{\delta}(h(t, x)) F_{2}(t, x)$ e $R_{\delta}(t, x, \varepsilon)=R_{1}(t, x, \varepsilon)+\phi_{\delta}(h(t, x)) R_{2}(t, x, \varepsilon)$.

Para cada $z \in C$, denote por $x(t, z, \varepsilon)$ a solução, no sentido de Filippov, do sistema 
(3.4.1), satisfazendo $x(0, t, \varepsilon)=z$. Analogamente, $x_{\delta}(t, z, \varepsilon)$ denota a solução de (3.4.2) tal que $x(0, z, \varepsilon)=z$. Como o sistema (3.4.2) é $T$-periódico na variável $t$, podemos considerá-lo definido no cilindro generalizado $\mathbb{S}^{1} \times D$. Este cilindro é obtido por uma identificação de $\Sigma=\{(\tau, x): \tau=0\} \operatorname{com}\{(\tau, x): \tau=T\}$. Dessa maneira poderemos aplicar o Teorema 3.1.11 e ter bem definida uma seção transversal $\Sigma$ ao fluxo (Ver Figura $3.1)$.

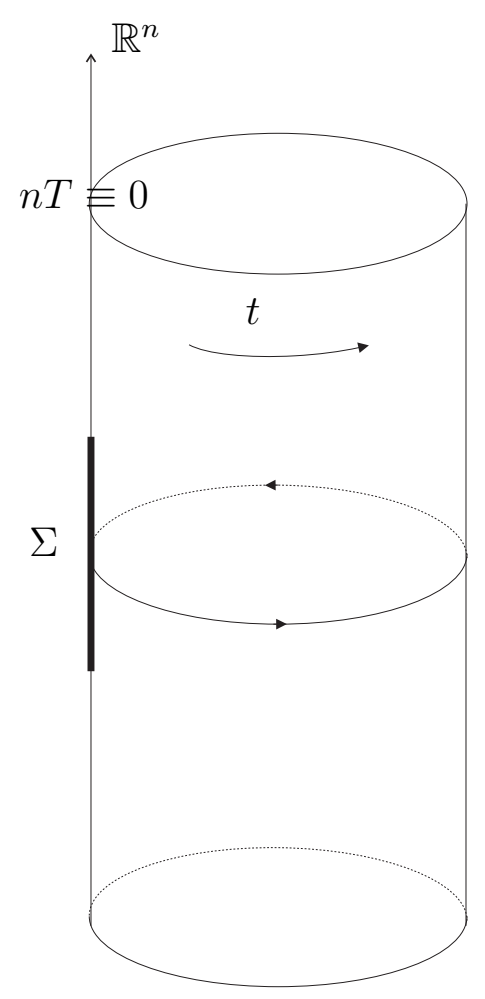

Figura 3.1: Cilindro generalizado

Note que neste cilindro, $\Sigma$ é uma seção transversal ao fluxo. Além disso, se $z \in C$ é a coordenada de um ponto em $\Sigma$, podemos considerar a aplicação de Poincaré $P_{\delta}^{\varepsilon}(z)=$ $x_{\delta}(T, z, \varepsilon)$, a qual sabemos estar bem definida para $\varepsilon \in\left[-\varepsilon_{0}, \varepsilon_{0}\right]$ onde $\varepsilon_{0}>0$.

Naturalmente, pela definição do mapa de Poincaré, existe relação entre ele e as órbitas periódicas de período $T$ do sistema (3.4.2). Analogamente, podemos definir o mapa de Poincaré para o sistema (3.4.1). Ele é dado por $P^{\varepsilon}(z)=x(T, z, \varepsilon)$. Mais à frente, provamos que essa aplicação é contínua.

Afirmação 3.4.1. Dado um compacto $K \subset D$, para $|\varepsilon|>0$ suficientemente pequeno, 
a órbita do sistema de Filippov (3.4.1), passando por $z \in K$, intercepta a variedade de descontinuidade em um número finito de pontos, para $0 \leq t \leq T$.

A órbita passando por $z \in K$ no cilindro generalizado é dada pela curva $\gamma_{\varepsilon}(t)=$ $(t, x(t, z, \varepsilon))$ que está definida para todo $t \in[0, T]$ e intercepta a variedade de descontinuidade somente em pontos da região de costura para $|\varepsilon|>0$ suficientemente pequeno. Suponhamos que exista uma sequência infinita(que podemos supor estritamente crescente) $\left(t_{i}\right)_{i \in \mathbb{N}} \subset[0, T]$ tal que

$$
h\left(t_{i}, x\left(t_{i}, z, \varepsilon\right)\right)=0 \quad \forall i \in \mathbb{N}
$$

Como $[0, T]$ é compacto, existe uma subsequência convergente $\left(t_{i_{j}}\right)_{j \in \mathbb{N}}$ tal que $t_{i_{j}} \rightarrow \bar{t} \in$ $[0, T]$. Dado que $\gamma_{\varepsilon}$ é contínua, segue que $\gamma_{\varepsilon}([0, T])$ é uma curva compacta e portanto é um conjunto fechado. Sendo assim, se denotarmos por $\bar{z}=x(\bar{t}, z, \varepsilon)$, teremos que $(\bar{t}, \bar{z}) \in \gamma_{\varepsilon}([0, T])$ (sendo fechada contém todos seus pontos de acumulação). Como $h$ é contínua, temos $h(\bar{t}, \bar{z})=0$ e portanto $(\bar{t}, \bar{z})$ é um ponto da variedade de descontinuidade do sistema. Por outro lado, $\bar{t}$ é ponto de acumulação e portanto dado $\varepsilon>0$, existe $j_{0}>0$ tal que $t_{i_{j}} \in(\bar{t}-\varepsilon, \bar{t}+\varepsilon)$ para todo $j \geq j_{0}$. Sendo assim, para $t \in(\bar{t}-\varepsilon, \bar{t}+\varepsilon)$, a órbita $\gamma_{\varepsilon}(t)$ intercepta a variedade de descontinuidade em um número infinito de pontos. O que não pode ocorrer, uma vez que $(\bar{t}, \bar{z})$ é um ponto na região de costura da variedade que é atingido pela órbita $\gamma_{\varepsilon}(t)$ e pela Proposição 3.3.11, a órbita $\gamma_{\varepsilon}(t)$ atravessa o ponto $(\bar{t}, \bar{z})$, ou seja, existe $\varepsilon_{0}>0$ tal que

$$
h(t, x(t, z, \varepsilon)) \neq 0, \quad \forall t \in\left(\bar{t}-\varepsilon_{0}, \bar{t}+\varepsilon_{0}\right) \backslash\{\bar{t}\} .
$$

Afirmação 3.4.2. Dado um subconjunto compacto $K \subset D$ para todo $|\varepsilon|>0$ suficientemente pequeno, $z \in K$ e $\bar{t} \in(0, T]$, tem-se que

$$
x(\bar{t}, z, \varepsilon)=z+\varepsilon \int_{0}^{\bar{t}} F(t, x(t, z, \varepsilon)) d t+\varepsilon^{2} \int_{0}^{\bar{t}} R(t, x(t, z, \varepsilon), \varepsilon) d t .
$$

De fato, pela Afirmação 3.4.1, para cada $z \in K$, podemos decompor a órbita passando por $z$ em finitas partes diferenciáveis: 


$$
x(t, z, \varepsilon)= \begin{cases}x_{1}(t, z, \varepsilon) & \text { se } 0=t_{0} \leq t \leq t_{1} \\ x_{2}(t, z, \varepsilon) & \text { se } t_{1} \leq t \leq t_{2} \\ \vdots & \\ x_{i}(t, z, \varepsilon) & \text { se } t_{i-1} \leq t \leq t_{i} \\ \vdots & \\ x_{k}(t, z, \varepsilon) & \text { se } t_{k-1} \leq t \leq t_{k}=T\end{cases}
$$

para as quais são válidas as seguintes relações

$$
x_{1}(0, z, \varepsilon)=z \quad \text { e } \quad x_{i+1}\left(t_{i}, z, \varepsilon\right)=x_{i}\left(t_{i}, z, \varepsilon\right), \quad i=1,2, \ldots, k-1 .
$$

Ou seja, as órbitas se "colam". Assim para cada $i=1,2, \ldots, k-1, x_{i}(t, z, \varepsilon)$ satisfaz a equação (3.3.2) e então se $t \in\left(t_{i}, t_{i+1}\right)$, temos

$$
\begin{aligned}
x_{i+1}(t, z, \varepsilon) & =x_{i+1}\left(t_{i}, z, \varepsilon\right)+\varepsilon \int_{t_{i}}^{t_{i+1}} F(t, x(t, z, \varepsilon)) d t+\varepsilon^{2} \int_{t_{i}}^{t_{i+1}} R(t, x(t, z, \varepsilon), \varepsilon) d t \\
& =x_{i}\left(t_{i}, z, \varepsilon\right)+\varepsilon \int_{t_{i}}^{t_{i+1}} F(t, x(t, z, \varepsilon)) d t+\varepsilon^{2} \int_{t_{i}}^{t_{i+1}} R(t, x(t, z, \varepsilon), \varepsilon) d t .
\end{aligned}
$$

Pela unicidade das soluções para sistemas de Filippov, segue que $P^{\varepsilon}(z)$ é escrito com composição de funções contínuas e portanto é contínua. Como existe $\bar{k} \in\{1,2, \ldots, k-1\}$ de modo que $\bar{t} \in\left(t_{\bar{k}}, t_{\bar{k}+1}\right)$, então

$$
\begin{aligned}
x(\bar{t}, z, \varepsilon) & =x_{\bar{k}}(\bar{t}, z, \varepsilon) \\
& =x_{\bar{k}-1}\left(t_{\bar{k}}, z, \varepsilon\right)+\varepsilon \int_{t_{\bar{k}}}^{t_{\bar{k}+1}} F(t, x(t, z, \varepsilon)) d t+\varepsilon^{2} \int_{t_{\bar{k}}}^{t_{\bar{k}+1}} R(t, x(t, z, \varepsilon), \varepsilon) d t
\end{aligned}
$$

podemos proceder por indução em $k$ para concluir a afirmação.

Observação 3.4.3. 1. Na Afirmação 3.4.2, provamos a continuidade em $C$ da aplicação de Poincaré para o sistema descontínuo (3.4.1), que por definição está diretamente relacionada com as órbitas periódicas do sistema. 
2. A hipótese ii) do Teorema 3.3.4 nos garante que para $|\varepsilon|>0$ suficientemente pequeno, as soluções que começam em $C$ cruzam o conjunto de descontinuidade somente em regiões de costura e portanto, pela Afirmação 3.4.2, $x_{\delta}(t, z, \varepsilon) \rightarrow x(t, z, \varepsilon)$ para todo $(t, z) \in[0, T] \times C$ e $|\varepsilon|>0$ suficientemente pequeno.

Pela Observação 3.4.3, para $z \in C$ e $|\varepsilon|>0$ suficientemente pequeno, temos:

$$
\lim _{\delta \rightarrow 0} P_{\delta}^{\varepsilon}(z)=P^{\varepsilon}(z), \quad \text { (limite pontual) }
$$

Observemos ainda que $f_{\delta}(z)=\int_{0}^{T} F_{\delta}(t, z) d t$ converge pontualmente à $f(z)=\int_{0}^{T} F(t, z) d t$ (para pontos de $\Sigma$ ) quando $\delta \rightarrow 0$.

Agora, seja $a \in U$ um ponto satisfazendo a hipótese $i i i)$ do Teorema 3.3.4. Então,

$$
f(z) \neq 0, \quad \forall z \in \bar{U} \backslash\{a\} .
$$

Definimos $f_{0}=f_{\left.\right|_{\bar{U}}}$. Pelo Lema 3.3.10, $f_{0}$ é contínua e então podemos considerar a seguinte homotopia contínua

$$
\left\{f_{\delta_{\mid \bar{U}}}: 0 \leq \delta \leq 1\right\}
$$

Afirmação 3.4.4. Existe $\delta_{0} \in(0,1]$ tal que $0 \notin f_{\delta}(\partial U)$, para todo $\delta \in\left[0, \delta_{0}\right]$.

Suponhamos que isso não ocorra, então existe uma sequência $\left(z_{m}\right)_{m \in \mathbb{N}} \subset \partial U$ tal que $f_{\frac{1}{m}}\left(z_{m}\right)=0$. Como $U$ é limitado, segue que $\partial U$ é compacto e então podemos extrair uma subsequência $\left(z_{m_{n}}\right)_{n \in \mathbb{N}}$ tal que $z_{m_{n}} \rightarrow z_{0} \in \partial U$. Como a função $f$ é contínua, segue que $f\left(z_{0}\right)=0$, contrariando a hipótese $\left.i i i\right)$.

Usando a propriedade da invariância por homotopia do grau de Brouwer, segue que $d_{B}\left(f_{\delta}, U, 0\right) \neq 0$ para $0 \leq \delta \leq \delta_{0}$. O que implica que existe $a_{\delta} \in U$ tal que $f_{\delta}\left(a_{\delta}\right)=0$. Note que essa raiz é isolada pois caso contário, como $f_{\delta} \rightarrow f$ pontualmente se $\delta \rightarrow 0$, temos que $a$ não é raiz isolada de $f$.

Aplicamos agora o Teorema 1.5.15 para garantir a existência de uma solução periódica $x_{\delta}(t, \varepsilon)$ do sistema $(3.4 .2)$ tal que $z_{(\delta, \varepsilon)} \doteq x_{\delta}(0, \varepsilon) \rightarrow a_{\delta}$ quando $\varepsilon \rightarrow 0$, ou seja, $z_{(\delta, \varepsilon)}$ é um ponto fixo do mapa de Poincaré $P_{\delta}^{\varepsilon}(z)$. 
Afirmação 3.4.5. O mapa de Poincaré $P^{\varepsilon}$ possui um ponto fixo.

Para $\varepsilon$ suficientemente pequeno, cada $z_{(\delta, \varepsilon)}$ é um ponto de alguma órbita periódica do sistema (3.4.2). Assim a sequência $\left(z_{\varepsilon}^{i}\right)_{i \in \mathbb{N}} \operatorname{com} z_{\varepsilon}^{i}=z_{\left(\frac{1}{i}, \varepsilon\right)}$ é limitada e portanto admite uma subsequência convergente $\left(z_{\varepsilon}^{i_{j}}\right)$ tal que $\left(z_{\varepsilon}^{i_{j}}\right) \rightarrow z_{\varepsilon}$ quando $j \rightarrow \infty$. Logo, $f_{\frac{1}{i_{j}}}\left(z_{\varepsilon}^{i_{j}}\right) \rightarrow f_{0}\left(z_{\varepsilon}\right)$ quando $j \rightarrow \infty$.

Mostremos que $P^{\varepsilon}\left(z_{\varepsilon}\right)=z_{\varepsilon}$. Dado que

$$
\begin{gathered}
z_{\varepsilon}=\lim _{j \rightarrow \infty} z_{\varepsilon}^{i_{j}}=\lim _{j \rightarrow \infty} P_{\frac{1}{i_{j}}}^{\varepsilon}\left(z_{\varepsilon}^{i_{j}}\right) \\
P^{\varepsilon}\left(z_{\varepsilon}\right)=P^{\varepsilon}\left(\lim _{j \rightarrow \infty} z_{\varepsilon}^{i_{j}}\right)=\lim _{j \rightarrow \infty} P^{\varepsilon}\left(z_{\varepsilon}^{i_{j}}\right),
\end{gathered}
$$

basta mostrar que

$$
\lim _{j \rightarrow \infty}\left(P^{\varepsilon}\left(z_{\varepsilon}^{i_{j}}\right)-P_{\frac{1}{i_{j}}}^{\varepsilon}\left(z_{\varepsilon}^{i_{j}}\right)\right)=0
$$

Sendo

$$
\begin{aligned}
\left\|P^{\varepsilon}\left(z_{\varepsilon}^{i_{j}}\right)-P_{\frac{1}{i_{j}}}^{\varepsilon}\left(z_{\varepsilon}^{i_{j}}\right)\right\| & =\left\|\varepsilon f_{0}\left(z_{\varepsilon}^{i_{j}}\right)-\varepsilon f_{\frac{1}{i_{j}}}\left(z_{\varepsilon}^{i_{j}}\right)+\mathcal{O}\left(\varepsilon^{2}\right)\right\| \\
& \leq \varepsilon\left\|f_{0}\left(z_{\varepsilon}^{i_{j}}\right)-f_{\frac{1}{i_{j}}}\left(z_{\varepsilon}^{i_{j}}\right)\right\|+\left\|\mathcal{O}\left(\varepsilon^{2}\right)\right\|,
\end{aligned}
$$

dado $\epsilon>0$ podemos escolher $j \in \mathbb{N}$ suficientemente grande e $\varepsilon$ suficientemente pequeno de modo que

$$
\left\|P^{\varepsilon}\left(z_{\varepsilon}^{i_{j_{k}}}\right)-P_{\frac{1}{i_{j_{k}}}}^{\varepsilon}\left(z_{\varepsilon}^{i_{j_{k}}}\right)\right\|<\epsilon
$$

Note que escolhemos o parâmetro $\varepsilon>0$ suficientemente pequeno afim de controlar o termo $\left\|\mathcal{O}\left(\varepsilon^{2}\right)\right\|$, enquanto que os demais termos são controlados por $j \in \mathbb{N}$.

Afirmação 3.4.6. $a_{\delta} \rightarrow$ a quando $\delta \rightarrow 0$.

De fato, suponha por absurdo o contrário. Considere a sequência $\left(a^{i}\right)_{i \in \mathbb{N}}$ tal que $a^{i}=a_{\frac{1}{i}}$ para cada $i \in \mathbb{N}$. Como $a_{\delta}$ não converge à $a$, existe um $\varepsilon_{0}>0$ e uma subsequência $\left(a^{i_{j}}\right)_{j \in \mathbb{N}}$ tal que

$$
\left\|a^{i_{j}}-a\right\| \geq \varepsilon_{0}
$$

Por outro lado, $\left(a^{i_{j}}\right)_{j \in \mathbb{N}}$ está contida em $U$ que é um subconjunto limitado e portanto a 
sequência $\left(a^{i_{j}}\right)$ é limitada . Logo ela admite uma subsequência $\left(a^{i_{j_{k}}}\right)_{k \in \mathbb{N}}$ convergente para $\bar{a} \in \bar{U} \backslash\{a\}$, quando $k \rightarrow \infty$. Logo,

$$
f_{\frac{1}{i_{j_{k}}}}\left(a^{i_{j_{k}}}\right) \rightarrow f(\bar{a}) \quad \text { quando } k \rightarrow \infty
$$

consequentemente $f(\bar{a})=0$, contrariando a hipótese iii) do teorema. Portanto $a_{\delta} \rightarrow a$.

Assim o sistema descontínuo (3.4.1) tem uma solução periódica $x(t, \varepsilon)$ de tal forma que $z_{\varepsilon}=x(0, \varepsilon) \rightarrow a$ se $\varepsilon \rightarrow 0$. Abaixo, um esquema representa as relações de convergência.

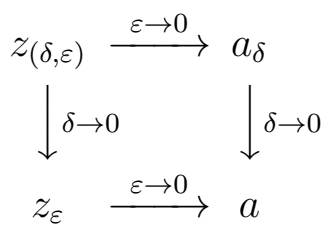




\title{
Capítulo
}

\section{Aplicações do averaging para alguns}

\author{
sistemas suaves por partes
}

Neste capítulo, apresentamos duas aplicações do Teorema 3.3.4.

A primeira aplicação apresentada foi desenvolvida por Llibre-Mereu em [25] para investigação dos sistemas com centros isócronos de uma dada forma normal. A segunda aplicação foi desenvolvida com base em trabalhos de Llibre-Mereu. Em [23] e [24] os autores investigam sobre o número de ciclos limites que bifurcam de um centro linear quando perturbado dentro de classes específicas de sistemas diferenciais planares, em [23] na classe dos sistemas diferenciais planares do tipo Kukles generalizado e em [24], quando o sistema é perturbado na classe dos sistemas suaves por partes do tipo Lienard com $n$ retas de descontinuidades.

Na segunda aplicação dessa dissertação, investigamos o número de ciclos limites que bifurcam de um centro linear quando perturbado numa classe de sistemas suaves por partes chamada tipo Kukles generalizado, que será introduzida na Seção 4.2. O resultado obtido é original. 


\subsection{Ciclos limites que bifurcam de um centro isócrono específico}

Exemplo 4.1.1. [25] O objetivo é investigar o número minimo de ciclos limites que bifurcam do centro isócrono

$$
\left\{\begin{array}{l}
\dot{x}=-y+x^{2} \\
\dot{y}=x+x y
\end{array}\right.
$$

quando perturbado na classe dos sistemas polinomiais quadráticos que possuem o eixo-x como variedade de descontinuidade.

Considere o seguinte sistema

$$
Z(x, y)= \begin{cases}Y_{1}(x, y) & \text { se } y>0 \\ Y_{2}(x, y) & \text { se } y<0\end{cases}
$$

onde $Y_{i}(x, y)=\left(\begin{array}{c}-y+x^{2}+\varepsilon p_{i}(x, y) \\ x+x y+\varepsilon q_{i}(x, y)\end{array}\right)$ para $\varepsilon$ suficientemente pequeno $e$

$$
\begin{aligned}
& p_{1}(x, y)=a_{1} x+a_{2} y+a_{3} x y+a_{4} x^{2}+a_{5} y^{2}, \\
& q_{1}(x, y)=b_{1} x+b_{2} y+b_{3} x y+b_{4} x^{2}+b_{5} y^{2}, \\
& p_{2}(x, y)=c_{1} x+c_{2} y+c_{3} x y+c_{4} x^{2}+c_{5} y^{2}, \\
& q_{2}(x, y)=d_{1} x+d_{2} y+d_{3} x y+d_{4} x^{2}+d_{5} y^{2} .
\end{aligned}
$$

Primeiramente vejamos como re-escrever o sistema na forma padrão. Considere o seguinte sistema planar

$$
\left\{\begin{array}{l}
\dot{x}=P(x, y) \\
\dot{y}=Q(x, y)
\end{array}\right.
$$

onde $P, Q: \mathbb{R}^{2} \rightarrow \mathbb{R}$ são funções contínuas. Assuma que o sistema tenha uma família de ovais

$$
\left\{\Gamma_{h}\right\} \subset\left\{(x, y): H(x, y)=h, h_{1}<h<h_{2}\right\},
$$


onde $H$ é uma integral primeira do sistema (4.1.2).

Perturbamos (4.1.2) como segue:

$$
\left\{\begin{array}{l}
\dot{x}=P(x, y)+\varepsilon p(x, y) \\
\dot{y}=Q(x, y)+\varepsilon q(x, y)
\end{array}\right.
$$

onde $p, q: \mathbb{R}^{2} \rightarrow \mathbb{R}$ são funções contínuas.

O próximo teorema será uma ferramenta útil para investigação do número de ciclos limites que bifurcam do sistema 4.1.1

Teorema 4.1.2. [11] Considere o sistema (4.1.2) e sua integral primeira H. Assumamos que $x Q(x, y)-y P(x, y) \neq 0$ para todo $(x, y)$ no período de anel formado pelas ovais $\left\{\Gamma_{h}\right\}$. Seja $\rho:\left(\sqrt{h_{1}}, \sqrt{h_{2}}\right) \times[0,2 \pi) \rightarrow[0, \infty)$ uma função continua tal que

$$
H(\rho(R, \varphi) \cos \varphi, \rho(R, \varphi) \operatorname{sen} \varphi)=R^{2}
$$

para todo $R \in\left(\sqrt{h_{1}}, \sqrt{h_{2}}\right)$ e todo $\varphi \in[0,2 \pi)$. Então, a equação diferencial que descreve a dependência entre a raiz quadrada da energia $R=\sqrt{h}$ e o ângulo $\varphi$ para o sistema (4.1.3) é dada por

$$
\frac{d R}{d \varphi}=\varepsilon \frac{\mu\left(x^{2}+y^{2}\right)(Q p-P q)}{2 R(Q x-P y)}+\mathcal{O}\left(\varepsilon^{2}\right)
$$

onde $\mu=\mu(x, y)$ é o fator integrante do sistema (4.1.2) correspondente à integral primeira $H$ e $x=\rho(R, \varphi) \cos \varphi$ e $y=\rho(R, \varphi) \operatorname{sen} \varphi$.

A proposição abaixo será importante para encontrar zeros de funções. Ele já era conhecido há algum tempo, mas sem demonstrações conhecidas. Como o resultado tornou-se muito útil para a técnica estudada, Llibre e Świrzcz demonstraram-no em [30].

Proposição 4.1.3. Se $f_{1}, f_{2}, \ldots, f_{n}: A \rightarrow \mathbb{R}$ são funçõs linearmente independentes então existem $a_{1}, a_{2}, \ldots, a_{n-1} \in A$ e $\alpha_{1}, \alpha_{2}, \ldots, \alpha_{n} \in \mathbb{R}$ tal que para todo $i \in\{1,2, \ldots, n-1\}$,

$$
\sum_{k=1}^{n} \alpha_{k} f_{k}\left(a_{i}\right)=0
$$


Com isso temos ferramentas suficientes para enunciar e demonstrar o seguinte resultado:

Teorema 4.1.4. [25] Para $|\varepsilon| \neq 0$ suficientemente pequeno, existem sistemas diferenciais polinomiais quadráticos suaves por partes com pelo menos 5 ciclos limites bifurcando das órbitas periódicas do centro isócrono

$$
\left\{\begin{array}{l}
\dot{x}=-y+x^{2}, \\
\dot{y}=x+x y .
\end{array}\right.
$$

Demonstração: Considere $H(x, y)=\frac{x^{2}+y^{2}}{(1+y)^{2}}$ e $\mu(x, y)=\frac{2}{(1+y)^{3}}$. H é uma integral primeira e $\mu$ é um fator integrante para o centro isócrono acima, relacionado à função $H$. De fato, observe primeiramente que $H$ está bem definida para $y \neq-1$ e o sistema (4.1.4) é invariante por $y=-1$ e portanto não temos problemas. Além disso $H$ é uma integral primeira se, e somente se, $P \frac{\partial H}{\partial x}+Q \frac{\partial H}{\partial y}=0$. No nosso caso,

$$
\begin{aligned}
P \frac{\partial H}{\partial x}+Q \frac{\partial H}{\partial y} & =\frac{2 x\left(-y+x^{2}\right)}{(1+y)^{2}}+\frac{2 y(1+y)-2 x\left(x^{2}+y^{2}\right)(1+y)}{(1+y)^{3}} \\
& =\frac{-2 x y+2 x^{3}+2 x y+2 x y^{2}-2 x^{3}-2 y^{2} x}{(1+y)^{2}}=0
\end{aligned}
$$

Agora, note que

$$
\mu P=\frac{2\left(-y+x^{2}\right)}{(1+y)^{3}}=-\frac{\partial H}{\partial y}
$$

$e$

$$
\mu Q=\frac{2(x+x y)}{(1+y)^{3}}=\frac{\partial H}{\partial x} .
$$

Logo, $\mu$ é fator integrante do sistema.

Nesse caso a família de ovais é dada por $\left\{(x, y) \in \mathbb{R}^{2} ; H(x, y)=h, 0<h<1\right\}$ e resolvendo a equação

$$
\frac{\rho^{2} \cos ^{2} \varphi+\rho^{2} \operatorname{sen}^{2} \varphi}{(1+\rho \operatorname{sen} \varphi)^{2}}=R^{2}
$$

na variável $\rho$, obtemos a função das hipóteses do Teorema 4.1.2, dada por:

$$
\rho(R, \varphi)=\frac{R}{1-R \operatorname{sen} \varphi}, \quad 0<R<1 \text { e } \varphi \in[0,2 \pi) .
$$


Fazendo $x=\rho(R, \varphi) \cos \varphi, y=\rho(R, \varphi) \operatorname{sen} \varphi$ re-escrevemos (4.1.1) na forma

$$
\frac{d R}{d \varphi}= \begin{cases}\varepsilon \frac{A(\varphi, a, b) R+B(\varphi, a, b) R^{2}+C(\varphi, a, b) R^{3}}{2(1-R \operatorname{sen} \varphi)}+\mathcal{O}\left(\varepsilon^{2}\right) & \text { se } y>0, \\ \varepsilon \frac{A(\varphi, c, d) R+B(\varphi, c, d) R^{2}+C(\varphi, c, d) R^{3}}{2(1-R \operatorname{sen} \varphi)}+\mathcal{O}\left(\varepsilon^{2}\right) & \text { se } y<0\end{cases}
$$

onde

$$
\begin{aligned}
A(\varphi, a, b) & =a_{1} \cos ^{2} \varphi+\left(a_{2}+b_{1}\right) \cos \varphi \operatorname{sen} \varphi+b_{2} \operatorname{sen}^{2} \varphi \\
B(\varphi, a, b) & =\left(a_{4}-b_{1}\right) \cos ^{3} \varphi+\left(-a_{1}+a_{3}-b_{2}+b_{4}\right) \cos ^{2} \varphi \operatorname{sen} \varphi \\
& +\left(-a_{2}+a_{5}-b_{1}+b_{3}\right) \cos \varphi \operatorname{sen}^{2} \varphi+\left(-b_{2}+b_{5}\right) \operatorname{sen}^{3} \varphi, \\
C(\varphi, a, b) & =-b_{4} \cos ^{4} \varphi+b_{1} \cos ^{3} \varphi \operatorname{sen} \varphi+\left(b_{2}-b_{4}-b_{5}\right) \cos ^{2} \varphi \operatorname{sen}^{2} \varphi \\
& +\left(b_{1}-b_{3}\right) \cos \varphi \operatorname{sen}^{3} \varphi+\left(b_{2}-b_{5}\right) \operatorname{sen}^{4} \varphi,
\end{aligned}
$$

para $a=\left(a_{1}, \ldots, a_{5}\right), b=\left(b_{1}, \ldots, b_{5}\right), c=\left(c_{1}, \ldots, c_{5}\right)$ e $d=\left(d_{1}, \ldots, d_{5}\right)$.

Observemos que com essa mudança de coordenadas, a função que dava origem à linha de descontinuidade $(h(x, y)=y)$ tornou-se $h(R, \varphi)=\frac{R \operatorname{sen} \varphi}{1-R \operatorname{sen} \varphi}$. Assim, $h(R, \varphi)>$ $0 \Leftrightarrow \operatorname{sen} \varphi>0$ e a função promediada para o sistema é dada por

$$
\begin{aligned}
f(R) & =\int_{0}^{\pi} \frac{A(\varphi, a, b) R+B(\varphi, a, b) R^{2}+C(\varphi, a, b) R^{3}}{2(1-R \operatorname{sen} \varphi)} d \varphi \\
& +\int_{\pi}^{2 \pi} \frac{A(\varphi, c, d) R+B(\varphi, c, d) R^{2}+C(\varphi, c, d) R^{3}}{2(1-R \operatorname{sen} \varphi)} d \varphi \\
& =\left(a_{1}+c_{1}\right) g_{1}+a_{3} g_{2}+b_{2} g_{3}+b_{4} g_{4}+b_{5} g_{5}+c_{3} g_{6}+d_{2} g_{7}+d_{4} g_{8}+d_{5} g_{9},
\end{aligned}
$$

onde

$$
\begin{aligned}
& g_{1}=\frac{\pi R}{4} \\
& g_{2}=1+\frac{\pi}{2 R}-\frac{\pi R}{4}+\frac{1}{4 R \sqrt{1-R^{2}}}\left(-2 \pi+2 \pi R^{2}-\left(4+4 R^{2}\right) \arcsin R\right) ; \\
& g_{3}=\frac{\pi R}{4}-R^{2}+\frac{1}{4 R \sqrt{1-R^{2}}}\left(2 \pi+4 \arccos h R-4 \arctan \frac{R}{\sqrt{1-R^{2}}}\right) ; \\
& g_{4}=1+\frac{\pi}{2 R}+\frac{\pi R}{4}-R^{2}+\frac{1}{4 R \sqrt{1-R^{2}}}\left(-2 \pi+4 \pi R^{2}-2 \pi R^{4}+\left(-4+8 R^{2}-4 R^{4}\right) \arcsin R\right) ;
\end{aligned}
$$




$$
\begin{aligned}
& g_{5}=g_{1}-g_{2}-g_{3} ; \\
& g_{6}=-1+\frac{\pi}{2 R}-\frac{\pi R}{4}-\frac{\sqrt{1-R^{2}} \arccos R}{R} \\
& g_{7}=2 g_{1}-g_{3} ; \\
& g_{8}=-1+\frac{\pi}{2 R}-\frac{3 \pi R}{4}+R^{2}+\left(R-\frac{1}{R}\right) \sqrt{1-R^{2}} \arccos R \\
& g_{9}=-g_{1}+g_{3}-g_{6} .
\end{aligned}
$$

Assim, $f$ pode ser escrita como

$f(R)=g_{1}\left(a_{1}+c_{1}+b_{5}+2 d_{2}-d_{5}\right)+g_{2}\left(a_{3}-b_{5}\right)+g_{3}\left(b_{2}-b_{5}-d_{2}+d_{5}\right)+b_{4} g_{4}+g_{6}\left(c_{3}-d_{5}\right)+d_{4} g_{8}$.

Expandindo em série de Taylor cada uma das funções $g_{i}, i=1,2,3,4,6,8$ temos

$$
\begin{aligned}
& g_{1}(R)=\frac{\pi}{4} R+\mathcal{O}\left(R^{7}\right) \\
& g_{2}(R)=\frac{1}{3} R^{2}+\frac{\pi}{16} R^{3}+\frac{2}{15} R^{4}+\frac{\pi}{32} R^{5}+\frac{8}{105} R^{6}+\mathcal{O}\left(R^{7}\right) \\
& g_{3}(R)=\frac{\pi}{4} R-R^{2}+\mathcal{O}\left(R^{7}\right) \\
& g_{4}(R)=\frac{1}{3} R^{2}-\frac{3 \pi}{16} R^{3}-\frac{1}{5} R^{4}-\frac{\pi}{32} R^{5}-\frac{2}{35} R^{6}+\mathcal{O}\left(R^{7}\right) \\
& g_{6}(R)=-\frac{1}{3} R^{2}+\frac{\pi}{16} R^{3}-\frac{2}{15} R^{4}+\frac{\pi}{32} R^{5}-\frac{8}{105} R^{6}+\mathcal{O}\left(R^{7}\right) \\
& g_{8}(R)=-\frac{1}{3} R^{2}-\frac{3 \pi}{16} R^{3}+\frac{1}{5} R^{4}-\frac{\pi}{32} R^{5}+\frac{2}{35} R^{6}+\mathcal{O}\left(R^{7}\right)
\end{aligned}
$$

$O$ determinante da matriz de coeficientes das variáveis $R, R^{2}, R^{3}, R^{4}, R^{6}$ é $-\frac{\pi^{3}}{33600} \neq 0$ e portanto as funções $g_{1}, g_{2}, g_{3}, g_{4}, g_{6}, g_{8}$ são linearmente independentes.

Pela Proposição 4.1.3 existe uma combinação linear dessas funções com pelo menos cinco zeros. Além disso os coeficientes das funções $g_{i}$, para $i=1,2,3,4,6,8$ na expressão de $f$ são funções lineares nas variáveis $a_{1}, a_{3}, b_{2}, b_{4}, b_{5}, c_{1}, c_{3}, d_{2}, d_{4}, d_{5}$. O posto da matriz jacobiana dos coeficientes de $g_{1}, g_{2}, g_{3}, g_{4}, g_{6}$ e $g_{8}$ em $f(R)$ nas variáveis $a_{1}, a_{3}, b_{2}, b_{4}, b_{5}, c_{1}$, $c_{3}, d_{2}, d_{4}, d_{5}$ é 6 . Portanto existem $R_{1}, R_{2}, R_{3}, R_{4}, R_{5}$ em $(0,1)$ e coeficientes $a_{j}, b_{j}, c_{j}, d_{j} \in$ $\mathbb{R}, j=1, \ldots, 5$, de tal forma que $f\left(R_{i}\right)=0$ para $i=1, \ldots, 5$. 
Aplicando o Teorema 3.3.4, garantimos que existem sistemas polinomiais diferenciais descontínuos quadráticos da forma (4.1.1) tendo pelo menos 5 ciclos limites bifurcando das órbitas periódicas do centro isócrono $\dot{x}=-y+x^{2}, \dot{y}=x+x y$.

\subsection{Sistemas diferenciais suaves por partes do tipo $\mathrm{Ku}-$} kles

Nesta seção vamos aplicar a teoria do averaging descontínuo de primeira ordem em uma classe de sistemas polinômiais diferenciais contínuos por partes "do tipo Kukles" para obter um limitante superior para o número de ciclos limites que essa família pode apresentar. O termo "do tipo Kukles" não é usual mas nós o adotamos neste trabalho afim de dar nome à classe de sistemas estudados.

O sistema clássico foi introduzido por Kukles em [22]. Ele deu condições necessárias e suficientes para que o sistema

$$
\begin{aligned}
& \dot{x}=-y, \\
& \dot{y}=x+a_{0} y+a_{1} x^{2}+a_{2} x y+a_{3} y^{2}+a_{4} x^{3}+a_{5} x^{2} y+a_{6} x y^{2}+a_{7} y^{3},
\end{aligned}
$$

possuísse um centro na origem. Usando o averaging contínuo de primeira e segunda ordem, Llibre e Mereu [23] estudaram o número máximo de ciclos limites que podem bifurcar das órbitas periódicas do centro linear $\dot{x}=-y, \dot{y}=x$, quando ele é perturbado dentro da classe de sistemas polinomiais diferenciáveis do tipo Kukles

$$
\begin{aligned}
& \dot{x}=y, \\
& \dot{y}=-x-\sum_{k \geq 1} \varepsilon^{k}\left(f_{n_{1}}^{k}(x)+g_{n_{2}}^{k}(x) y+h_{n_{3}}^{k}(x) y^{2}+d^{k} y^{3}\right),
\end{aligned}
$$

onde para todo $k$ os polinômios $f_{n_{1}}^{k}, g_{n_{2}}^{k}, h_{n_{3}}^{k}$ têm grau $n_{1}, n_{2}$ e $n_{3}$ respectivamente, $d^{k} \neq 0$ é um número real e $\varepsilon$ é um parâmetro pequeno.

Aqui vamos trabalhar com retas de descontinuidade passando através da origem do $\mathbb{R}^{2}$ e com dois sistemas diferenciais contínuos do tipo Kukles (na forma de (4.2.1)) localizados 
de forma alternada nos setores definidos pelas retas. Essa ideia foi aplicada para estudar uma classe de sistemas descontínuos generalizados do tipo Lienard [24].

Seja $l$ um natural diferente de zero e considere a função $h_{l}: \mathbb{R}^{2} \rightarrow \mathbb{R}$

$$
h_{l}(x, y)=\prod_{k=0}^{l-1}\left(y-\tan \left(\alpha+\frac{k \pi}{l}\right) x\right)
$$

O conjunto $h_{l}^{-1}(0)$ é o produto de $l$ retas passando pela origem e divide o plano em $2 l$ setores de ângulo $\frac{\pi}{l}$ quando $\alpha \in\left(-\frac{\pi}{l}, \frac{\pi}{l}\right)$.

Vamos investigar um limitante superior para o número de ciclos limites que podem bifurcar das óbitas periódicas do centro linear $\dot{x}=y, \dot{y}=-x$ quando ele é perturbado como segue

$$
\dot{X}= \begin{cases}X_{1}(x, y) & \text { se } h(x, y)>0 \\ X_{2}(x, y) & \text { se } h(x, y)<0\end{cases}
$$

onde $X_{j}(x, y)=\left(\begin{array}{c}y \\ -x-\varepsilon\left(f_{n_{1}}^{j}(x)+g_{n_{2}}^{j}(x) y+h_{n_{3}}^{j}(x) y^{2}+d_{0}^{j} y^{3}\right)\end{array}\right) \operatorname{com} f_{n_{1}}^{j}(x), g_{n_{2}}^{j}(x) \mathrm{e}$ $h_{n_{3}}^{j}(x)$ polinômios de grau $n_{1}, n_{2}$ e $n_{3}$ respectivamente, e $d_{0}^{j}$ é uma constante real não nula para $j=1,2$, quando $l \in\{1,2,3\}$.

O próximo resultado fornece uma estimativa para o número de ciclos limites do sistema descontínuo (4.2.3) sob determinadas condições.

Teorema 4.2.1. Assuma que para $j=1,2$, os polinômios $f_{n_{1}}^{j}(x), g_{n_{2}}^{j}(x)$ e $h_{n_{3}}^{j}(x)$ têm grau $n_{1} \geq 1, n_{2} \geq 1$ e $n_{3} \geq 1$ respectivamente, $d_{0}^{j}$ é uma constante não nula e $l \in\{1,2,3\}$. Então para $|\varepsilon|$ suficientemente pequeno o número máximo de ciclos limites do sistema diferencial generalizado descontínuo do tipo Kukles (4.2.3) que bifurcam do centro linear $\dot{x}=y, \dot{y}=-x$, pela teoria do averaging, é $m(l)$, onde

$$
\begin{aligned}
& \text { i) } m(1)=\max \left\{2\left[\frac{n_{1}}{2}\right], n_{2}+1,2\left[\frac{n_{3}+2}{2}\right], 3\right\} ; \\
& \text { ii) } m(2)=\max \left\{\left[\frac{n_{1}-1}{2}\right],\left[\frac{n_{2}}{2}\right],\left[\frac{n_{3}+1}{2}\right], 1\right\} ; \\
& \text { iii) } m(3)=\max \left\{2\left[\frac{n_{1}}{2}\right], n_{2}+1,2\left[\frac{n_{3}+2}{2}\right], 3\right\}-1 .
\end{aligned}
$$


Em [23] Llibre e Mereu mostraram que os sistemas diferenciais polinomiais generalizados do tipo Kukles têm no máximo $\max \left\{\left[\frac{n_{2}}{2}\right], 1\right\}$ ciclos limites bifurcando do centro linear $\dot{x}=y, \dot{y}=-x$ pela teoria do averaging. Quando introduzimos retas de descontinuidade nessa mesma classe de sistemas o número de ciclos limites aumenta. Para uma reta de descontinuidade $(l=1)$ existem pelo menos dois ciclos limites a mais do que o caso suave. Para $l=3$ existe pelo menos um ciclo limite a mais enquanto que para $l=2$ o número de ciclos limites é o mesmo ou maior, dependendo da escolha de $n_{1}, n_{2}$ e $n_{3}$, inteiros que definem o grau da perturbação.

\section{Demonstração do Teorema 4.2.1}

Escrevendo

$$
\begin{array}{rlrl}
f_{n_{1}}^{1}(x) & =\sum_{i=0}^{n_{1}} a_{i} x^{i}, & g_{n_{2}}^{1}(x)=\sum_{i=0}^{n_{2}} b_{i} x^{i}, & h_{n_{3}}^{1}(x)=\sum_{i=0}^{n_{3}} c_{i} x^{i}, \\
f_{n_{1}}^{2}(x)=\sum_{i=0}^{n_{1}} d_{i} x^{i}, & g_{n_{2}}^{2}(x)=\sum_{i=0}^{n_{2}} e_{i} x^{i}, & h_{n_{3}}^{2}(x)=\sum_{i=0}^{n_{3}} m_{i} x^{i},
\end{array}
$$

fazendo a mudança de coordenadas $x=r \cos \theta, y=r \operatorname{sen} \theta$ e tomando $\theta$ como a nova variável independente, o sistema (4.2.3) se escreve na forma

$$
\frac{d r}{d \theta}=\varepsilon \operatorname{sen} \theta P_{j}(r, \theta)+\mathcal{O}\left(\varepsilon^{2}\right)
$$

onde $P_{1}$ e $P_{2}$ são dadas por

$$
\begin{aligned}
P_{1}(r, \theta) & =\sum_{i=0}^{n_{1}} a_{i} r^{i} \cos ^{i} \theta+\sum_{i=0}^{n_{2}} b_{i} r^{i+1} \cos ^{i} \theta \operatorname{sen} \theta \\
& +\sum_{i=0}^{n_{3}} c_{i} r^{i+2} \cos ^{i} \theta \operatorname{sen}^{2} \theta+d_{0}^{1} r^{3} \operatorname{sen}^{3} \theta \\
P_{2}(r, \theta) & =\sum_{i=0}^{n_{1}} d_{i} r^{i} \cos ^{i} \theta+\sum_{i=0}^{n_{2}} e_{i} r^{i+1} \cos ^{i} \theta \operatorname{sen} \theta \\
& +\sum_{i=0}^{n_{3}} m_{i} r^{i+2} \cos ^{i} \theta \operatorname{sen}^{2} \theta+d_{0}^{2} r^{3} \operatorname{sen}^{3} \theta
\end{aligned}
$$


Observe que o sistema (4.2.4) satisfaz as condições do Teorema $3.3 .4 \mathrm{com} h_{l}$ dada por (4.2.2). Assim, para estimar o número de ciclos limites do sistema (4.2.3), precisamos estimar o número de zeros da função promediada (3.3.3).

Definindo as funções

$$
\begin{aligned}
& \varphi_{i j l}(\alpha) \doteq \sum_{k=1}^{l} \int_{\alpha+\frac{2(k-1) \pi}{l}}^{\alpha+\frac{(2 k-1) \pi}{l}} \cos ^{i} \theta \operatorname{sen}^{j} \theta d \theta \\
& \bar{\varphi}_{i j l}(\alpha) \doteq \sum_{k=1}^{l} \int_{\alpha+\frac{(2 k-1) \pi}{l}}^{\alpha+\frac{2 k \pi}{l}} \cos ^{i} \theta \operatorname{sen}^{j} \theta d \theta
\end{aligned}
$$

a função promediada (3.3.3) é escrita como

$$
\begin{aligned}
f(r) & =\sum_{i=0}^{n_{1}} r^{i}\left[a_{i} \varphi_{i 1 l}(\alpha)+d_{i} \bar{\varphi}_{i 1 l}(\alpha)\right]+\sum_{i=0}^{n_{2}} r^{i+1}\left[b_{i} \varphi_{i 2 l}(\alpha)+e_{i} \bar{\varphi}_{i 2 l}(\alpha)\right] \\
& +\sum_{i=0}^{n_{3}} r^{i+2}\left[c_{i} \varphi_{i 3 l}(\alpha)+m_{i} \bar{\varphi}_{i 3 l}(\alpha)\right]+r^{3}\left[d_{0}^{1} \varphi_{04 l}(\alpha)+d_{0}^{2} \bar{\varphi}_{04 l}(\alpha)\right] .
\end{aligned}
$$

O próximo lema exibe uma relação entre algumas integrais na expressão da função promediada.

Lema 4.2.2. Para cada $i \in \mathbb{N}$ e $l \neq 0$ vale

a) $\varphi_{i 1 l}=-\bar{\varphi}_{i 1 l}$,

b) $\varphi_{i 3 l}=-\bar{\varphi}_{i 3 l}$.

c) Se ié impar então $\varphi_{i 2 l}=-\bar{\varphi}_{i 2 l}$.

Demonstração: Note que para cada $l \neq 0$

$$
\begin{aligned}
\left(\varphi_{i 1 l}+\bar{\varphi}_{i 1 l}\right)(\alpha) & =\sum_{k=1}^{l}\left(\int_{\alpha+\frac{2(k-1) \pi}{l}}^{\alpha+\frac{(2 k-1) \pi}{l}} \cos ^{i} \theta \operatorname{sen} \theta d \theta+\int_{\alpha+\frac{(2 k-1) \pi}{l}}^{\alpha+\frac{2 k \pi}{l}} \cos ^{i} \theta \operatorname{sen} \theta d \theta\right) \\
& =\sum_{k=1}^{l} \int_{\alpha+\frac{2(k-1) \pi}{l}}^{\alpha+\frac{2 k \pi}{l}} \cos ^{i} \theta \operatorname{sen} \theta d \theta=\int_{\alpha}^{\alpha+2 \pi} \cos ^{i} \theta \operatorname{sen} \theta d \theta=0 .
\end{aligned}
$$


Análogamente

$$
\begin{aligned}
\left(\varphi_{i 3 l}+\bar{\varphi}_{i 3 l}\right)(\alpha) & =\sum_{k=1}^{l}\left(\int_{\alpha+\frac{2(k-1) \pi}{l}}^{\alpha+\frac{(2 k-1) \pi}{l}} \cos ^{i} \theta \operatorname{sen}^{3} \theta d \theta+\int_{\alpha+\frac{(2 k-1) \pi}{l}}^{\alpha+\frac{2 k \pi}{l}} \cos ^{i} \theta \operatorname{sen}^{3} \theta d \theta\right) \\
& =\sum_{k=1}^{l} \int_{\alpha+\frac{2(k-1) \pi}{l}}^{\alpha+\frac{2 k \pi}{l}} \cos ^{i} \theta \operatorname{sen}^{3} \theta d \theta=\int_{\alpha}^{\alpha+2 \pi} \cos ^{i} \theta \operatorname{sen}^{3} \theta d \theta=0 .
\end{aligned}
$$

Esses cálculos provam os itens $a$ ) e $b)$.

Como $\left(\varphi_{i 2 l}+\bar{\varphi}_{i 2 l}\right)(\alpha)=\int_{\alpha}^{\alpha+2 \pi} \cos ^{i} \theta \operatorname{sen}^{2} \theta d \theta$, assumindo $i=2 m+1$ o item $\left.c\right)$ segue diretamente da fórmula 2.511-4 de [15]

$$
\begin{aligned}
& \int \cos ^{2 m+1} \theta \operatorname{sen}^{2} \theta d \theta= \\
& =\frac{\operatorname{sen}^{3} \theta}{2 m+3}\left(\sum_{j=1}^{m} \frac{2^{j} m(m-1) \ldots(m-j+1)}{(2 m+1)(2 m-1) \ldots(2 m-2 j+3)} \cos ^{2 m-2 j} \theta+\cos ^{2 m} \theta\right) .
\end{aligned}
$$

Observação 4.2.3. Note que

1) se $i$ é par $\varphi_{i 2 l}(\alpha), \bar{\varphi}_{i 2 l}(\alpha)>0$ para todo $l \neq 0$ e $\alpha \in \mathbb{R}$; de fato, se $i$ é par então $\cos ^{i} \theta \operatorname{sen}^{2} \theta \geq 0$.

2) $\varphi_{04 l}(\alpha), \bar{\varphi}_{04 l}(\alpha)>0$ para todo $\alpha \in \mathbb{R}$ pois $\operatorname{sen}^{4} \theta \geq 0$.

A fim de estimarmos os zeros da função promediada resta-nos estudar três funções: $\varphi_{i 1 l}, \varphi_{i 3 l}$ e $\varphi_{i 2 l}$. As duas primeiras para cada $i \in \mathbb{N}$ e $l \neq 0$ e a terceira para $i$ ímpar. Para um estudo detalhado dessas funções precisamos fixar o número $l \neq 0$.

\section{Demonstração do item (i)}

Seja $\alpha \in\left(-\frac{\pi}{2}, \frac{\pi}{2}\right)$. O caso $\alpha=\frac{\pi}{2}$ será considerado separadamente. Se $l=1$ então $h_{1}(x, y)=y-(\tan \alpha) x$ e $h_{1}^{-1}(0)=\left\{(x, y) \in \mathbb{R}^{2}: y=(\tan \alpha) x\right\}$.

Os próximos lemas serão usados para estimar o número máximo de zeros da função promediada (4.2.5) quando $l=1$.

Lema 4.2.4. Se $i$ é ímpar então $\varphi_{i 11} \equiv 0$ e $\varphi_{i 11}$ não se anula se $\alpha \neq \pm \frac{\pi}{2}$ e $i$ é par. 
Demonstração: Agora, note que

$$
\begin{aligned}
\varphi_{i 11}(\alpha) & =\int_{\alpha}^{\alpha+\pi} \cos ^{i} \theta \text { sen } \theta d \theta=-\frac{(-1)^{i+1} \cos ^{i+1} \alpha-\cos ^{i+1} \alpha}{i+1} \\
& = \begin{cases}0 & \text { se } i \text { é ímpar, } \\
2 \frac{\cos ^{i+1} \alpha}{i+1} & \text { se } i \text { é par. }\end{cases}
\end{aligned}
$$

Então se $i$ é par a função $\varphi_{i 11}(\alpha)$ se anula se e somente se $\alpha= \pm \pi / 2$.

Lema 4.2.5. Se $i$ é ímpar e $\alpha \neq 0$ então as funções $\varphi_{i 21}$ não se anulam.

Demonstração: Se $i=1$ então

$$
\varphi_{121}(\alpha)=\int_{\alpha}^{\alpha+\pi} \operatorname{sen}^{2} \theta \cos \theta d \theta=-\frac{2}{3} \operatorname{sen}^{3} \alpha
$$

Portanto, se $\alpha \neq 0$, a função $\varphi_{121}$ não se anula.

Se $i \geq 1$ e ímpar podemos escrever $i=2 m+1$ para algum $m \in \mathbb{N} \backslash\{0\}$ e de (4.2.6) obtemos

$$
\begin{aligned}
& \varphi_{(2 m+1) 21}(\alpha)=\int_{\alpha}^{\alpha+\pi} \operatorname{sen}^{2} \theta \cos ^{2 m+1} \theta d \theta= \\
& -\frac{\operatorname{sen}^{3} \alpha}{2 m+3}\left(\cos ^{2 m} \alpha+\sum_{j=1}^{m} \frac{2^{j} m(m-1) \ldots(m-j+1)}{(2 m+1)(2 m-1) \ldots(2 m-2 j+3)} \cos ^{2 m-2 j} \alpha\right) \\
& -\left(\frac{\operatorname{sen}^{3} \alpha}{2 m+3}\left(\cos ^{2 m} \alpha+\sum_{j=1}^{m} \frac{2^{j} m(m-1) \ldots(m-j+1)}{(2 m+1)(2 m-1) \ldots(2 m-2 j+3)} \cos ^{2 m-2 j} \alpha\right)\right) \\
& =-2 \frac{\operatorname{sen}^{3} \alpha}{2 m+3}\left(\sum_{j=1}^{m} \frac{2^{j} m(m-1) \ldots(m-j+1)}{(2 m+1)(2 m-1) \ldots(2 m-2 j+3)} \cos ^{2 m-2 j} \alpha\right. \\
& \left.+\cos ^{2 m} \alpha\right) .
\end{aligned}
$$

Assim, $\varphi_{(2 m+1) 20}(\alpha)=0$ se e somente se $\alpha=0$.

Lema 4.2.6. Considerando $i$ par e $\alpha \neq \pm \frac{\pi}{2}$, a função $\varphi_{i 31}$ não se anula. Além disso $\varphi_{i 31} \equiv 0$ quando $i$ é ímpar. 
Demonstração: Temos que

$$
\begin{aligned}
\varphi_{i 31}(\alpha) & =\int_{\alpha}^{\alpha+\pi} \cos ^{i} \theta \operatorname{sen}^{3} \theta d \theta \\
& =\frac{(-1)^{i+3} \cos ^{i+3} \alpha-\cos ^{i+3} \alpha}{i+3}-\frac{(-1)^{i+1} \cos ^{i+1} \alpha-\cos ^{i+1} \alpha}{i+1} \\
& = \begin{cases}0 & \text { se } i \text { é ímpar, } \\
2\left(\frac{\cos ^{i+1} \alpha}{i+1}-\frac{\cos ^{i+3} \alpha}{i+3}\right) \neq 0 & \text { se } i \text { é par. }\end{cases}
\end{aligned}
$$

Assim, a função $\varphi_{i 31}(\alpha)$ é zero se e somente se $i$ é ímpar ou $\alpha= \pm \frac{\pi}{2}$.

Prova do Teorema 4.2.1 (i). Pelos lemas anteriores a função promediada é dada por

$$
\begin{aligned}
f(r) & =\sum_{\substack{i=0 \\
i \text { par }}}^{n_{1}} A_{i}(\alpha)\left(a_{i}-d_{i}\right) r^{i}+\sum_{\substack{i=0 \\
i \text { par }}}^{n_{2}} B_{i}(\alpha)\left(b_{i}+e_{i}\right) r^{i+1}+\sum_{\substack{i=1 \\
i \text { impar }}}^{n_{2}} C_{i}(\alpha)\left(b_{i}-e_{i}\right) r^{i+1} \\
& +\sum_{\substack{i=0 \\
i \text { par }}}^{n_{3}} D_{i}(\alpha)\left(c_{i}-m_{i}\right) r^{i+2}+\left(E^{1}(\alpha) d_{0}^{1}+E^{2}(\alpha) d_{0}^{2}\right) r^{3}
\end{aligned}
$$

com $A_{i}(\alpha)$ e $D_{i}(\alpha)$ diferente de zero se $\alpha \neq \pm \frac{\pi}{2}, B_{i}(\alpha), E^{1}(\alpha), E^{2}(\alpha)>0$, e $C_{i}(\alpha) \neq 0$ se $\alpha \neq 0$.

Então o grau da função promediada é $m=m(1)$, onde $m(1)$ é dado por

(i) $\max \left\{n_{2}+1,3\right\}$, se $\alpha \in\left\{-\frac{\pi}{2}, \frac{\pi}{2}\right\}$;

(ii) $\max \left\{2\left[\frac{n_{1}}{2}\right], 2\left[\frac{n_{2}}{2}\right]+1,2\left[\frac{n_{3}+2}{2}\right], 3\right\}$, se $\alpha=0$;

(iii) $\max \left\{2\left[\frac{n_{1}}{2}\right], n_{2}+1,2\left[\frac{n_{3}+2}{2}\right], 3\right\}$, se $\alpha \notin\left\{0, \pm \frac{\pi}{2}\right\}$,

e portanto o número máximo de zeros da função promediada será $m$ em cada caso.

Como os polinômios $1, r, r^{2}, r^{3}, \ldots, r^{m}$ são funções linearmente independentes podemos aplicar a Proposição 4.1.3 e o mesmo raciocínio usado no Exemplo 4.1.1 para garantir que é possível escolher os coeficientes $a_{i}, b_{i}, c_{i}, d_{i}, e_{i}, m_{i}$ e $d_{0}^{j}$ e $\alpha$ na expressão da função promediada de tal forma que $f(r)$ tenha exatamente $m(1)=\max \left\{2\left[\frac{n_{1}}{2}\right], n_{2}+1,2\left[\frac{n_{3}+2}{2}\right], 3\right\}$ raízes positivas simples, o que garante que o número máximo de zeros é atingido. Após 
tais escolhas, aplicamos o Teorema 3.3.4 para garantir que o sistema (4.2.3) possui no máximo $m(1)$ ciclos limites, cada um com período próximo de $2 \pi$. Isso prova o item (i) do Teorema 4.2.1.

\section{Demonstração do item (ii)}

Para $l=2$ em (4.2.2) temos $h_{2}(x, y)=(y-(\tan \alpha) x)\left(y+\tan \left(\alpha+\frac{\pi}{2}\right) x\right)$. O grau de (4.2.5) quando $l=2$ será determinado usando os próximos lemas.

Lema 4.2.7. A função $\varphi_{i 12}$ não se anula para $\alpha \neq \pm \frac{\pi}{4}$ e $i$ impar. Se $i$ é par então $\varphi_{i 12} \equiv 0$.

Demonstração: Temos

$$
\begin{aligned}
\varphi_{i 12}(\alpha) & =\int_{\alpha}^{\alpha+\frac{\pi}{2}} \cos ^{i} \theta \operatorname{sen} \theta d \theta+\int_{\alpha+\pi}^{\alpha+\frac{3 \pi}{2}} \cos ^{i} \theta \operatorname{sen} \theta d \theta \\
& = \begin{cases}0 & \text { se } i \text { é par, } \\
2 \frac{\cos ^{i+1} \alpha-\operatorname{sen}^{i+1} \alpha}{i+1} & \text { se } i \text { é ímpar. }\end{cases}
\end{aligned}
$$

É fácil ver que $\varphi_{112}(\alpha)=0$ se e somente se $\cos ^{2} \alpha=\operatorname{sen}^{2} \alpha$, isto é, se e somente se, $\alpha= \pm \frac{\pi}{4}, \pm \frac{3 \pi}{4}$.

Se $i \geq 1$ é um natural ímpar então $i+1=2 n$ para algum $n \in \mathbb{N}$ e

$$
\begin{gathered}
\left(\cos ^{2 n-2} \alpha+\sum_{k+j=2(n-1)} \cos ^{k} \alpha \operatorname{sen}^{j} \alpha+\operatorname{sen}^{2 n-2} \alpha\right)\left(\cos ^{2} \alpha-\operatorname{sen}^{2} \alpha\right) \\
=\cos ^{2 n} \alpha-\operatorname{sen}^{2 n} \alpha .
\end{gathered}
$$

Assim, os zeros da função $\varphi_{i 12}(\alpha)$ são $\alpha=\frac{\pi}{4}, \frac{3 \pi}{4}$ no intervalo estabelecido.

Lema 4.2.8. Se $i$ é impar a função $\varphi_{i 22}$ é identicamente nula.

Demonstração: Se $i=1$ então

$$
\varphi_{122}(\alpha)=\frac{1}{3}\left(\cos ^{3} \alpha-\operatorname{sen}^{3} \alpha\right)+\frac{1}{3}\left(-\cos ^{3} \alpha+\operatorname{sen}^{3} \alpha\right)=0
$$


Se $i \geq 1$ é ímpar então usamos a fórmula (4.2.6) para escrever

$$
\begin{aligned}
\varphi_{i 22}(\alpha) & =\frac{\operatorname{sen}^{3}\left(\alpha+\frac{\pi}{2}\right)}{2 m+3}\left(\cos ^{2 m}\left(\alpha+\frac{\pi}{2}\right)+\sum_{j=1}^{m} A(m, j) \cos ^{2 m-2 j}\left(\alpha+\frac{\pi}{2}\right)\right) \\
- & \frac{\operatorname{sen}^{3} \alpha}{2 m+3}\left(\cos ^{2 m} \alpha+\sum_{j=1}^{m} A(m, j) \cos ^{2 m-2 j} \alpha\right) \\
+ & \frac{\operatorname{sen}^{3}\left(\alpha+\frac{3 \pi}{2}\right)}{2 m+3}\left(\cos ^{2 m}\left(\alpha+\frac{3 \pi}{2}\right)+\sum_{j=1}^{m} A(m, j) \cos ^{2 m-2 j}\left(\alpha+\frac{3 \pi}{2}\right)\right) \\
- & \frac{\operatorname{sen}^{3}(\alpha+\pi)}{2 m+3}\left(\cos ^{2 m}(\alpha+\pi)+\sum_{j=1}^{m} A(m, j) \cos ^{2 m-2 j}(\alpha+\pi)\right)
\end{aligned}
$$

onde $A(m, j)=\frac{2^{j} m(m-1) \ldots(m-j+1)}{(2 m+1)(2 m-1) \ldots(2 m-2 j+3)}$.

Assim,

$$
\varphi_{i 22}(\alpha)=0
$$

Lema 4.2.9. Se $i$ é ímpar, os únicos zeros da função $\varphi_{i 32}$ são $\alpha= \pm \frac{\pi}{4}$. Quando $i$ é par a função é identicamente nula.

Demonstração: Com cálculos diretos podemos observar $\varphi_{i 32}(\alpha) \equiv 0$, quando $i$ é par. Caso contrário,

$$
\varphi_{i 32}(\alpha)=\frac{2}{i+3}\left(\operatorname{sen}^{i+3} \alpha-\cos ^{i+3} \alpha\right)+\frac{2}{i+1}\left(-\operatorname{sen}^{i+1} \alpha+\cos ^{i+1} \alpha\right)
$$

Além disso, $\alpha= \pm \frac{\pi}{4}$ são zeros da função $\varphi_{i 32}(\alpha)$ quando $i$ é ímpar. A fim de mostrar que essas são as únicas raízes no intervalo estabelecido, estudaremos o sinal de sua derivada

$$
\begin{aligned}
\varphi_{i 32}^{\prime}(\alpha) & =\frac{2}{i+3}\left((i+3) \operatorname{sen}^{i+2} \alpha \cos \alpha+(i+3) \cos ^{i+2} \alpha \operatorname{sen} \alpha\right) \\
& +\frac{2}{i+1}\left((i+1) \operatorname{sen} \alpha \cos ^{i} \alpha-(i+1) \cos \alpha \operatorname{sen}^{i} \alpha\right) \\
& =-2\left(\cos ^{i} \alpha \operatorname{sen}^{3} \alpha+\cos ^{3} \alpha \operatorname{sen}^{i} \alpha\right)
\end{aligned}
$$

Se $\alpha \in\left(-\frac{\pi}{2}, 0\right), \varphi_{i 32}^{\prime}(\alpha)>0$ e então $\varphi_{i 32}(\alpha)$ é estritamente crescente. Para $\alpha \in\left(0, \frac{\pi}{2}\right)$, 
$\varphi_{i 32}^{\prime}(\alpha)<0$ e então $\varphi_{i 32}(\alpha)$ é estritamente decrescente. Assim, os únicos zeros de $\varphi_{i 32}(\alpha)$ no intervalo estabelecido são $\alpha= \pm \frac{\pi}{4}$.

Prova do Teorema 4.2.1 (ii). Segue dos Lemas 4.2.7 - 4.2.9 que a função promediada é dada por

$$
\begin{aligned}
f(r) & =\sum_{\substack{i=1 \\
i \text { ímpar }}}^{n_{1}} A_{i}(\alpha)\left(a_{i}-d_{i}\right) r^{i}+\sum_{\substack{i=0 \\
i \text { par }}}^{n_{2}}\left(B_{i}(\alpha) b_{i}+\bar{B}_{i}(\alpha) e_{i}\right) r^{i+1} \\
& +\sum_{\substack{i=1 \\
i \text { mimpar }}}^{n_{3}} C_{i}(\alpha)\left(c_{i}-m_{i}\right) r^{i+2}+\left(E^{1}(\alpha) d_{0}^{1}+E^{2}(\alpha) d_{0}^{2}\right) r^{3},
\end{aligned}
$$

onde $A_{i}(\alpha)$ e $C_{i}(\alpha)$ nunca se anulam exceto em $\alpha= \pm \frac{\pi}{4}$ e $B_{i}(\alpha), \bar{B}_{i}(\alpha)$ não possuem zeros.

A função promediada $f(r)$ é um polinômio em $r$ de grau $m=m(2)$ onde $m(2)$ é dado por

1) $\max \left\{2\left[\frac{n_{1}-1}{2}\right]+1,2\left[\frac{n_{2}}{2}\right]+1,2\left[\frac{n_{3}+1}{2}\right]+1,3\right\}$, se $\alpha \notin\left\{-\frac{\pi}{4}, \frac{\pi}{4}\right\}$;

2) $\max \left\{2\left[\frac{n_{2}}{2}\right]+1,3\right\}$ caso contrário.

Além disso, se $\alpha \neq \pm \frac{\pi}{4}$ a função promediada é uma função ímpar e portanto possui no máximo $\frac{m-1}{2}$ raízes positivas. Do Teorema 3.3.4 segue a prova do Teorema 4.2.1 (ii), como feito na demonstração do item (i).

\section{Demonstração do item (iii)}

Quando $l=3$ em (4.2.2) a função $h_{3}$ é dada por

$$
h_{3}(x, y)=(y-(\tan \alpha) x)\left(y-\left(\tan \left(\alpha+\frac{2 \pi}{3}\right)\right) x\right)\left(y-\left(\tan \left(\alpha+\frac{4 \pi}{3}\right)\right) x\right) .
$$

Lema 4.2.10. A respeito da função $\varphi_{i 13}$, as seguintes afirmações são válidas:

(i) $\varphi_{i 13}^{\prime}(\alpha)<0$ em $\left(0, \frac{\pi}{3}\right)$.

(ii) $\varphi_{i 13}(\alpha)$ é uma função par. 
(iii) $\varphi_{i 13}(\alpha)$ é $2 \pi / 3-$ periódica.

(iv) $\varphi_{i 13}(\alpha) \neq 0$, se $\alpha \neq \pm \frac{\pi}{6}$ e $i \neq 0$ é par.

Demonstração: Temos:

$$
\begin{aligned}
\varphi_{i 13}(\alpha) & =\int_{\alpha}^{\alpha+\frac{\pi}{3}} \cos ^{i} \theta \operatorname{sen} \theta d \theta+\int_{\alpha+\frac{2 \pi}{3}}^{\alpha+\pi} \cos ^{i} \theta \operatorname{sen} \theta d \theta+\int_{\alpha+\frac{4 \pi}{3}}^{\alpha+\frac{5 \pi}{3}} \cos ^{i} \theta \operatorname{sen} \theta d \theta \\
= & \frac{(-1)^{i} \cos ^{i+1} \alpha+\cos ^{i+1} \alpha-(-1)^{i+1} \operatorname{sen}^{i+1}\left(\alpha-\frac{\pi}{6}\right)+\operatorname{sen}^{i+1}\left(\alpha-\frac{\pi}{6}\right)}{i+1} \\
& +\frac{(-1)^{i+1} \operatorname{sen}^{i+1}\left(\alpha+\frac{\pi}{6}\right)-\operatorname{sen}^{i+1}\left(\alpha+\frac{\pi}{6}\right)}{i+1} .
\end{aligned}
$$

Assim, $\varphi_{i 13}(\alpha)$ se anula se $i$ é ímpar. Se $i$ é par então

$$
\varphi_{i 13}(\alpha)=\frac{2}{i+1}\left(\cos ^{i+1} \alpha+\operatorname{sen}^{i+1}\left(\alpha-\frac{\pi}{6}\right)-\operatorname{sen}^{i+1}\left(\alpha+\frac{\pi}{6}\right)\right)
$$

Portanto $\varphi_{013}(\alpha) \equiv 0$. A fim de considerarmos o caso $i$ par não nulo, derivamos $\varphi_{i 13}$ em relação à $\alpha$. Usando ferramentas computacionais, como o Mathematica, obtemos

$$
\begin{aligned}
\varphi_{i 13}^{\prime}(\alpha) & =\left(-2 \cos ^{i} \alpha \operatorname{sen} \alpha-(\sqrt{3} \cos \alpha+\operatorname{sen} \alpha)\right. \\
& \left.\left((\sqrt{3} \operatorname{sen} \alpha+\cos \alpha)^{i}-(\sqrt{3} \operatorname{sen} \alpha-\cos \alpha)^{i}\right) / 2^{i}\right) .
\end{aligned}
$$

A menos de menção contrária, os cálculos abaixo serão feitos no intervalo $\left(0, \frac{\pi}{3}\right)$.

(a) $\operatorname{sen} \alpha \cos ^{i} \alpha>0$;

(b) $\sqrt{3} \cos \alpha+\operatorname{sen} \alpha>0$ 
(c) $(\cos \alpha+\sqrt{3} \operatorname{sen} \alpha)^{i}-(-\cos \alpha+\sqrt{3} \operatorname{sen} \alpha)^{i}=$

$$
\begin{aligned}
= & \sum_{\substack{k=1 \\
k \text { impar }}}^{i-1}\left(\begin{array}{c}
i \\
k
\end{array}\right)(\sqrt{3})^{k} \cos ^{i-k} \alpha \operatorname{sen}^{k} \alpha+\sum_{\substack{k=2 \\
k \text { par }}}^{i-2}\left(\begin{array}{l}
i \\
k
\end{array}\right)(\sqrt{3})^{k} \cos ^{i-k} \alpha \operatorname{sen}^{k} \alpha \\
+ & \sum_{\substack{k=1 \\
k \text { impar }}}^{i-1}\left(\begin{array}{l}
i \\
k
\end{array}\right)(\sqrt{3})^{k} \cos ^{i-k} \alpha \operatorname{sen}^{k} \alpha-\sum_{\substack{k=2 \\
k \text { par }}}^{i-2}\left(\begin{array}{c}
i \\
k
\end{array}\right)(\sqrt{3})^{k} \cos ^{i-k} \alpha \operatorname{sen}^{k} \alpha \\
= & 2 \sum_{\substack{k=1 \\
k \text { impar }}}^{i-1}\left(\begin{array}{c}
i \\
k
\end{array}\right)(\sqrt{3})^{k} \cos ^{i-k} \alpha \operatorname{sen}^{k} \alpha>0 .
\end{aligned}
$$

Portanto $\varphi_{i 13}^{\prime}(\alpha)<0$ se $\alpha \in\left(0, \frac{\pi}{3}\right)$, concluindo que $\varphi_{i 13}(\alpha)$ é estritamente decrescente neste intervalo. O item (i) está provado.

Além disso,

$$
\begin{aligned}
\varphi_{i 13}(-\alpha) & =\frac{2}{i+1}\left(\cos ^{i+1}(-\alpha)+\operatorname{sen}^{i+1}\left(-\alpha-\frac{\pi}{6}\right)-\operatorname{sen}^{i+1}\left(-\alpha+\frac{\pi}{6}\right)\right) \\
& =\frac{2}{i+1}\left(\cos ^{i+1}(\alpha)-\operatorname{sen}^{i+1}\left(\alpha+\frac{\pi}{6}\right)+\operatorname{sen}^{i+1}\left(\alpha-\frac{\pi}{6}\right)\right) \\
& =\varphi_{i 13}(\alpha)
\end{aligned}
$$

de onde concluímos a prova do item (ii), i.e., $\varphi_{i 13}(\alpha)$ é uma função par.

Dado que

$$
\begin{aligned}
\varphi_{i 13}\left(\alpha+\frac{2 \pi}{3}\right) & =\frac{2}{i+1}\left(\cos ^{i+1}\left(\alpha+\frac{2 \pi}{3}\right)-\operatorname{sen}^{i+1}\left(\alpha+\frac{5 \pi}{6}\right)+\operatorname{sen}^{i+1}\left(\alpha-\frac{\pi}{2}\right)\right) \\
& =\frac{2}{i+1}\left(-\operatorname{sen}^{i+1}\left(\alpha+\frac{\pi}{6}\right)+\operatorname{sen}^{i+1}\left(\alpha-\frac{\pi}{2}\right)-\operatorname{sen}^{i+1}\left(\alpha+\frac{5 \pi}{6}\right)\right. \\
& =\frac{2}{i+1}\left(\cos ^{i+1}(\alpha)-\operatorname{sen}^{i+1}\left(\alpha+\frac{\pi}{6}\right)+\operatorname{sen}^{i+1}\left(\alpha-\frac{\pi}{6}\right)\right) \\
& =\varphi_{i 13}(\alpha),
\end{aligned}
$$

segue que $\varphi_{i 13}(\alpha)$ é $\frac{2 \pi}{3}$-periódica.

Finalmente, se $i \neq 0$

$$
\varphi_{i 13}(0)=\frac{2^{1-i}}{i+1}\left(2^{i}-1\right) \neq 0
$$

Na Figura 4.1 é possível ver o gráfico de $\varphi_{i 13}(\alpha)$, para $i$ par diferente de zero. 


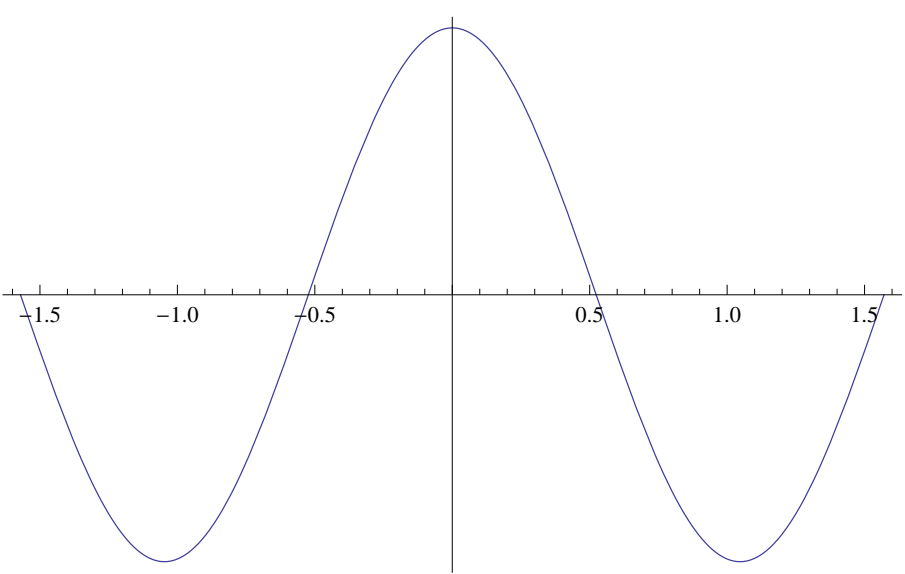

Figura 4.1: Gráfico de $\varphi_{i 13}(\alpha)$, para $i \neq 0$ par

Como $\varphi_{i 13}$ é estritamente decrescente em $\left(0, \frac{\pi}{3}\right)$ e $\varphi_{i 13}\left(\frac{\pi}{6}\right)=0$ segue que $\frac{\pi}{6}$ é o único zero de $\varphi_{i 13}$ em $\left(0, \frac{\pi}{3}\right)$. Analogamente, como $\varphi_{i 13}$ é estritamente crescente em $\left(-\frac{\pi}{3}, 0\right),-\frac{\pi}{6}$ é o único zero neste intervalo.

Agora, suponha que exista um $\alpha_{0} \in\left(\frac{\pi}{3}, \frac{\pi}{2}\right)$ tal que $\varphi_{i 13}\left(\alpha_{0}\right)=0$. Portanto $\varphi_{i 13}\left(\alpha_{0}-\right.$ $\left.\frac{2 \pi}{3}\right)=0$. Mas se $\alpha_{0} \in\left(\frac{\pi}{3}, \frac{\pi}{2}\right)$, então $\alpha_{0}-\frac{2 \pi}{3} \in\left(-\frac{\pi}{3},-\frac{\pi}{6}\right)$ será um zero de $\varphi_{i 13}$, absurdo.

Lema 4.2.11. Se $i$ é impar e $\alpha \notin\left\{0, \pm \frac{\pi}{3}\right\}$ então a função $\varphi_{i 23}$ não se anula.

Demonstração: Se $i=1$ então $\varphi_{123}(\alpha)=\frac{1}{2} \operatorname{sen}(3 \alpha)$. Caso contrário, escrevemos $i=$ $2 m+1$ para algum $m \in \mathbb{N} \backslash\{0\}$ e usando (4.2.6) obtemos

$$
\begin{aligned}
& \varphi_{i 23}(\alpha)= \\
& =2\left[\frac{\operatorname{sen}^{3}\left(\alpha+\frac{\pi}{3}\right)}{2 m+3}\left(\cos ^{2 m}\left(\alpha+\frac{\pi}{3}\right)+\sum_{j=1}^{m} A(m, j) \cos ^{2 m-2 j}\left(\alpha+\frac{\pi}{3}\right)\right)\right. \\
& +\frac{\operatorname{sen}^{3}\left(\alpha+\frac{5 \pi}{3}\right)}{2 m+3}\left(\cos ^{2 m}\left(\alpha+\frac{5 \pi}{3}\right)+\sum_{j=1}^{m} A(m, j) \cos ^{2 m-2 j}\left(\alpha+\frac{5 \pi}{3}\right)\right) \\
& \left.+\frac{\operatorname{sen}^{3}(\alpha+\pi)}{2 m+3}\left(\cos ^{2 m}(\alpha+\pi)+\sum_{j=1}^{m} A(m, j) \cos ^{2 m-2 j}(\alpha+\pi)\right)\right]
\end{aligned}
$$

$\operatorname{com} A(m, j)=\frac{2^{j} m(m-1) \ldots(m-j+1)}{(2 m+1)(2 m-1) \ldots(2 m-2 j+3)}$.

Usando o software Mathematica avaliamos as expressões de $\varphi_{i 23}(\alpha) \operatorname{com} i=2 m+1$, para alguns valores de $m$. Abaixo, listamos essas expressões $(m=1,2, \ldots, 7)$, 
(i) $\varphi_{323}(\alpha)=1 / 8 \operatorname{sen}(3 \alpha)$,

(ii) $\varphi_{523}(\alpha)=-61 / 1.120 \operatorname{sen}(3 \alpha)$,

(iii) $\varphi_{723}(\alpha)=-31 / 630 \operatorname{sen}(3 \alpha)+1 / 384 \operatorname{sen}(9 \alpha)$,

(iv) $\varphi_{923}(\alpha)=-26.123 / 887.040 \operatorname{sen}(3 \alpha)+7 / 1.536 \operatorname{sen}(9 \alpha)$,

(v) $\varphi_{11,23}(\alpha)=(1 / 92.252 .160)(-1.612 .309 \operatorname{sen}(3 \alpha)+389.550 \operatorname{sen}(9 \alpha))$,

(vi) $\varphi_{13,23}(\alpha)=(1 / 5.535 .129 .600)(-60.534 .421 \operatorname{sen}(3 \alpha)+$ 17.493.735 $\operatorname{sen}(9 \alpha)+135.135 \operatorname{sen}(15 \alpha))$,

(vi) $\varphi_{15,23}(\alpha)=(1 / 376.388 .812 .800)(-2.715 .648 .724 \operatorname{sen}(3 \alpha)+$ $811.055 .280 \operatorname{sen}(9 \alpha)+29.864 .835 \operatorname{sen}(15 \alpha))$.

De suas expressões conclui-se que $\varphi_{i 23}(\alpha)=a_{m} \operatorname{sen}(3 \alpha)+b_{m} \operatorname{sen}(9 \alpha)+c_{m} \operatorname{sen}(15 \alpha)+\ldots$, com $a_{m}, b_{m}, c_{m} \in \mathbb{R}$. Portanto os zeros dessa função são $0,-\frac{\pi}{3}, \frac{\pi}{3}$.

Seguindo o raciocínio do caso $l=2$ pode-se provar o seguinte Lema:

Lema 4.2.12. A função $\varphi_{i 33}$ não se anula para $\alpha \neq \pm \frac{\pi}{6}$ e $i$ par. Se $i$ é ímpar então $\varphi_{i 33} \equiv 0$.

Prova do Teorema 4.2.1 (iii). Dos Lemas 4.2.10 - 4.2.12 concluímos que, no caso $l=3$ a função promediada possui a seguinte expressão

$$
\begin{aligned}
f(r) & =\sum_{\substack{i=2 \\
i \text { par }}}^{n_{1}} r^{i} A_{i}(\alpha)\left(a_{i}-d_{i}\right)+\sum_{\substack{i=0 \\
i \text { par }}}^{n_{2}} r^{i+1}\left(b_{i} B_{i}(\alpha)+e_{i} \bar{B}_{i}(\alpha)\right) \\
& +\sum_{\substack{i=1 \\
i \text { impar }}}^{n_{2}} r^{i+1} C_{i}(\alpha)\left(b_{i}-e_{i}\right)+\sum_{\substack{i=2 \\
i \text { par }}}^{n_{3}} r^{i+2} D_{i}(\alpha)\left(c_{i}-m_{i}\right) \\
& +\left(E^{1}(\alpha) d_{0}^{1}+E^{2}(\alpha) d_{0}^{2}\right) r^{3}
\end{aligned}
$$

onde $B_{i}(\alpha), \bar{B}_{i}(\alpha)>0$ para todo $\alpha, C_{i}(\alpha)$ não se anula se $\alpha \notin\left\{0, \pm \frac{\pi}{3}\right\}$ e $A_{i}(\alpha), D_{i}(\alpha)$ são diferente de zero se $\alpha \neq \pm \frac{\pi}{6}$.

Assim, $f(r)$ é uma função polinomial de grau $m=m(3)$, onde 
1) $m(3)=\max \left\{2\left[\frac{\mathrm{n}_{1}}{2}\right], 2\left[\frac{\mathrm{n}_{2}}{2}\right]+1,2\left[\frac{\mathrm{n}_{3}+2}{2}\right], 3\right\}$ se $\alpha \in\left\{0,-\frac{\pi}{3}, \frac{\pi}{3}\right\}$.

2) $m(3)=\max \left\{\mathrm{n}_{2}+1,3\right\}$ se $\alpha \in\left\{-\frac{\pi}{6}, \frac{\pi}{6}\right\}$

3) $m(3)=\max \left\{2\left[\frac{\mathrm{n}_{1}}{2}\right], \mathrm{n}_{2}+1,2\left[\frac{\mathrm{n}_{3}+2}{2}\right], 3\right\}$ se $\alpha \notin\left\{0, \pm \frac{\pi}{6}, \pm \frac{\pi}{3}\right\}$.

Aplicando o Teorema 3.3.4 como feito anteriormente, concluímos que $m(3)$ é um limitante superior para o número de ciclos limites do sistema (4.2.3) quando $l=3$.

Lembramos que é possível escolher os coeficientes da perturbação de tal forma que a função promediada $f(r)$ tenha exatamente $m$ raízes positivas. Portanto, como discutido anteriormente, a prova do Teorema 4.2.1 está concluída.

\subsubsection{Exemplos}

Nesta seção, ilustraremos o Teorema 4.2.1 pelo estudo da existência de soluções periódicas para três sistemas diferenciais generalizados contínuos por partes do tipo Kukles.

Exemplo 4.2.13 (Uma linha de descontinuidade). Considere $l=1, \alpha=0$ e as funções

$$
\begin{array}{ll}
f_{n_{1}}^{1}(x)=24+x+x^{2}, & f_{n_{1}}^{2}(x)=12+2 x-\frac{103}{2} x^{2}, \\
g_{n_{2}}^{1}(x)=2 x+x^{2}, & g_{n_{2}}^{2}(x)=-\frac{100}{\pi}+x-\frac{(80+\pi)}{\pi} x^{2}, \\
h_{n_{3}}^{1}(x)=1+3 x+15 x^{2}, & h_{n_{3}}^{2}(x)=1-3 x-\frac{45}{4} x^{2} .
\end{array}
$$

Tome as constantes $d_{0}^{1}=1$ e $d_{0}^{2}=-1$. Sob essas condições temos $n_{1}=n_{2}=n_{3}=2$, $m=4$ e o seguinte sistema descontinuo:

$$
Z(x, y)= \begin{cases}X_{1}(x, y) & \text { se } h(x, y)>0 \\ X_{2}(x, y) & \text { se } h(x, y)<0\end{cases}
$$

onde $h(x, y)=y, X_{1}(x, y)=\left(\begin{array}{c}y \\ -x-\varepsilon F_{1}(x, y)\end{array}\right) e X_{2}(x, y)=\left(\begin{array}{c}y \\ -x-\varepsilon F_{2}(x, y)\end{array}\right)$ sendo $F_{1}(x, y)=24+x+x^{2}+\left(2 x+x^{2}\right) y+\left(1+3 x+15 x^{2}\right) y^{2}+y^{3} e$

$$
F_{2}(x, y)=12+2 x-\frac{103}{2} x^{2}+\left(-\frac{100}{\pi}+x-\frac{(80+\pi)}{\pi} x^{2}\right) y+\left(1-3 x-\frac{45}{4} x^{2}\right) y^{2}-y^{3} .
$$


Portanto a função promediada é dada por

$$
\begin{aligned}
f(r) & =24 \int_{0}^{\pi} \operatorname{sen} \theta d \theta+12 \int_{\pi}^{2 \pi} \operatorname{sen} \theta d \theta-\frac{100}{\pi} r \int_{\pi}^{2 \pi} \operatorname{sen}^{2} \theta d \theta+ \\
& +r^{2}\left(\int_{0}^{\pi} \cos ^{2} \theta \operatorname{sen} \theta d \theta+\int_{0}^{\pi} \operatorname{sen}^{3} \theta d \theta+\int_{\pi}^{2 \pi} \operatorname{sen}^{3} \theta d \theta+\right. \\
& \left.-\frac{103}{2} \int_{\pi}^{2 \pi} \operatorname{sen} \theta \cos ^{2} \theta d \theta\right)+r^{3}\left(\int_{0}^{\pi} \cos ^{2} \theta \operatorname{sen}^{2} \theta d \theta+\right. \\
& \left.+\int_{0}^{\pi} \operatorname{sen}^{4} \theta d \theta-\frac{(80+\pi)}{\pi} \int_{\pi}^{2 \pi} \operatorname{sen}^{2} \theta \cos ^{2} \theta d \theta\right)+ \\
& +r^{4}\left(15 \int_{0}^{\pi} \cos ^{2} \theta \operatorname{sen}^{3} \theta d \theta+\frac{45}{4} \int_{\pi}^{2 \pi} \operatorname{sen}^{3} \theta \cos ^{2} \theta d \theta\right)= \\
& =r^{4}-10 r^{3}+35 r^{2}-50 r+24,
\end{aligned}
$$

cujas raízes são $r=1,2,3,4$. Pelo Teorema 3.3.4 segue que para $\varepsilon \neq 0$ suficientemente pequeno o sistema diferencial descontínuo (4.2.7) tem quatro ciclos limites.

Exemplo 4.2.14 (Duas linhas de descontinuidade). Considere $l=2, \alpha=0$, as funções

$$
\begin{array}{ll}
f_{n_{1}}^{1}(x)=4+2 x-3 x^{2}+2 x^{3}, & f_{n_{1}}^{2}(x)=7+3 x+9 x^{2}+2 x^{3}, \\
g_{n_{2}}^{1}(x)=3+5 x, & g_{n_{2}}^{2}(x)=-3+2 x, \\
h_{n_{3}}^{1}(x)=9+x, & h_{n_{3}}^{2}(x)=5-x .
\end{array}
$$

Tomando as constantes $d_{0}^{1}=1$ e $d_{0}^{2}=-1$, temos $n_{1}=3, n_{2}=n_{3}=1$ e $m=3$. Assim, obtemos o seguinte sistema descontínuo

$$
Z(x, y)= \begin{cases}X_{1}(x, y) & \text { se } h(x, y)>0 \\ X_{2}(x, y) & \text { se } h(x, y)<0\end{cases}
$$

onde $h(x, y)=y, X_{1}(x, y)=\left(\begin{array}{c}y \\ -x-\varepsilon F_{1}(x, y)\end{array}\right)$ e $X_{2}(x, y)=\left(\begin{array}{c}y \\ -x-\varepsilon F_{2}(x, y)\end{array}\right)$, sendo $F_{1}(x, y)=4+2 x-3 x^{2}+2 x^{3}+(3+5 x) y+(9+x) y^{2}+y^{3} e$

$$
F_{2}(x, y)=7+3 x+9 x^{2}+2 x^{3}+(-3+2 x) y+(5-x) y^{2}-y^{3} .
$$


A função promediada desse sistema é dada por

$$
f(r)=r^{3}-r
$$

cujas raízes são $r=0,1,-1$. Portanto, pelo Teorema 3.3.4 segue que para $\varepsilon \neq 0$ suficientemente pequeno, o sistema diferencial contínuo por partes (4.2.8) possui um ciclo limite.

Exemplo 4.2.15 (Três linhas de descontinuidade). Considere $l=3, \alpha=\frac{\pi}{4}$, as funções

$$
\begin{array}{ll}
f_{n_{1}}^{1}(x)=3+2 x+x^{2}, & f_{n_{1}}^{2}(x)=-2+7 x+x^{2}, \\
g_{n_{2}}^{1}(x)=2 x, & g_{n_{2}}^{2}(x)=-\frac{12}{\pi}+(2-22 \sqrt{2}) x, \\
h_{n_{3}}^{1}(x)=1+3 x+2 x^{2}, & h_{n_{3}}^{2}(x)=1+2 x+(2+8 \sqrt{2}) x^{2},
\end{array}
$$

e as constantes $d_{0}^{1}=d_{0}^{2}=-\frac{8}{\pi}$. Nesse caso, $n_{1}=n_{3}=2, n_{2}=1, m=4$ e o sistema descontinuos é

$$
Z(x, y)= \begin{cases}X_{1}(x, y) & \text { se } h(x, y)>0 \\ X_{2}(x, y) & \text { se } h(x, y)<0\end{cases}
$$

onde $h(x, y)=y, X_{1}(x, y)=\left(\begin{array}{c}y \\ -x-\varepsilon F_{1}(x, y)\end{array}\right)$ e $X_{2}(x, y)=\left(\begin{array}{c}y \\ -x-\varepsilon F_{2}(x, y)\end{array}\right)$, com $F_{1}(x, y)=3+2 x+x^{2}+2 x y+\left(1+3 x+2 x^{2}\right) y^{2}-\frac{8}{\pi} y^{3} e$

$$
F_{2}(x, y)=-2+7 x+x^{2}+\left(-\frac{12}{\pi}+(2-22 \sqrt{2}) x\right) y+\left(1+2 x+(2+8 \sqrt{2}) x^{2}\right) y^{2}-\frac{8}{\pi} y^{3} .
$$

A função promediada é dada por

$$
f(r)=r^{4}-6 r^{3}+11 r^{2}-6 r
$$

cujas raízes são $r=0,1,2,3$. Assim, pelo Teorema 3.3.4 segue que para $\varepsilon \neq 0$ suficientemente pequeno o sistema descontínuo (4.2.9) possui três ciclos limites. 


\subsubsection{Conjectura}

Através do estudo dos casos com uma, duas e três retas de descontinuidade, concluímos que a estimativa do grau da função promediada se torna complicada a medida que o número de retas aumenta e até onde chegamos não fomos capazes de estabelecer uma expressão geral para ela em função do número de retas $l$. Entretanto, observando algumas particularidades chegamos à seguinte conjectura.

Conjectura 4.2.16. Assuma que $j=1,2$, os polinômios $f_{n_{1}}^{j}(x), g_{n_{2}}^{j}(x)$ e $h_{n_{3}}^{j}(x)$ possuam grau $n_{1} \geq 1, n_{2} \geq 1$ e $n_{3} \geq 1$ respectivamente, $d_{0}^{j}$ é uma constante não nula e $l \in \mathbb{N}$. Então para $|\varepsilon|$ suficientemente pequeno o sistema diferencial generalizado descontínuo do tipo Kukles (4.2.3) possui no máximo $m(l)$ ciclos limites, onde

$$
\begin{aligned}
& \text { i) } m(l)=\max \left\{2\left[\frac{n_{1}}{2}\right], n_{2}+1,2\left[\frac{n_{3}+2}{2}\right], 3\right\} \text { se l é impar; } \\
& \text { ii) } m(l)=\max \left\{\left[\frac{n_{1}-1}{2}\right],\left[\frac{n_{2}}{2}\right],\left[\frac{n_{3}+1}{2}\right], 1\right\} \text { se l é par; }
\end{aligned}
$$

Observação 4.2.17. Note que no Exemplo 4.1.1 a teoria do averaging nos permite concluir a existência de uma cota inferior para o número de ciclos limites do sistema diferencial, enquanto que o Teorema 4.2.1 exibe uma cota superior. Essa diferença ocorre pelo fato de que a função promediada obtida no Exemplo 4.1.1 não é polinomial e não conseguimos estimar seu número máximo de zeros. Em contrapartida esse número pode ser encontrado para a família de sistemas diferenciais descontínuos do tipo Kukles, dado que a função promediada é uma função polinomial. 


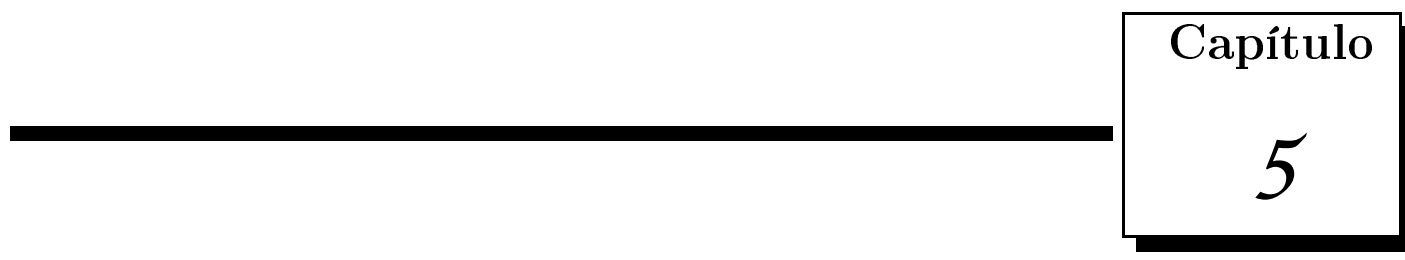

\section{Considerações finais}

Nessa dissertação estudamos a teoria do averaging desenvolvida para a investigação sobre o número de ciclos limites que bifurcam de um centro quando perturbados em classes de sistemas com descontinuidades. Apresentamos ainda duas aplicações dessa técnica, sendo uma delas motivada e obtida pela generalização de trabalhos anteriores de Llibre e Mereu, para uma classe de sistemas diferenciais planares que chamamos do tipo Kukles generalizado, para detalhes ver Capítulo 4.

Além disso, durante o desenvolvimento dessa dissertação, tivemos oportunidade de trabalhar com outras recentes generalizações da teoria do averaging, que extendem a teoria para outras classes de sistemas diferenciais tanto contínuos quanto descontínuos e estão apresentados em [26] e [27]. Essa investigação ocorreu durante visita do professor Jaume Llibre ao ICMC-USP, com apoio do projeto PVE, Ciência sem Fronteiras. Esse estudo, concluído recentemente, não está descrito nessa dissertação, mas todos os detalhes podem ser encontrados no trabalho disponível em

http://icmc.usp.br/CMS/Arquivos/arquivos_enviados/ESTAGIO-BIBLIO_171_Serie \%20Mat\%20399.pdf.

Nossos planos são dar continuidade ao trabalho iniciado no mestrado durante o doutorado, onde pretendemos estudar bifurcação de conjuntos minimais através de perturbações contínuas e descontínuas de sistemas reversíveis. A obtenção de técnicas adequadas a esse estudo bem como suas aplicações são nossos objetivos. 


\section{Referências Bibliográficas}

[1] O. B. Almeida, Teoria do grau e aplicações. Dissertação (Mestrado em Matemática) - CCT/UFCG, Campina Grande, (2006).

[2] A. A. Andronov, A.A. Vitt, S. E. Khaikin, Theory of oscillators, International Series of Monographs In Physics 4 (1966), Pergamon Press.

[3] V. I. ARNOL'D, Loss of stability of self-oscillations close to resonance and versal deformations of equivariant vector fields, Funct. Anal. Appl. 11 (1977), 85-92.

[4] V. I. ARnoL'D, Mathematical methods of classical mechanics. Graduate Texts in Mathematics, 60. New York: Springer-Verlag, 1989.

[5] V. I. ARnol'D, Ten problems, Adv. Soviet. Math. 1 (1990), 1-8.

[6] J. C. Artés, F. Dumortier, J. Llibre, Qualitative theory of planar differential systems. New York: Springer, 2006.

[7] E. A. Barbashin, Introduction to the Theory of Stability (T. Lukes, Ed.), Noordhoff, Groningen, 1970.

[8] A. D. Bazykin, Nonlinear Dynamics of Interacting Populations, River-Edge, NJ: World Scientific, (1998).

[9] B. Brogliato, Nonsmooth Mechanics, New York: Springer-Verlag, 1999.

[10] F. Browder, Fixed point theory and nonlinear problems, Bull. Amer. Math. Soc. 9 (1983), 1-39. 
[11] A. BuiC̆ degree, Bull. Sci. Math. 128 (2004), 7-22.

[12] J. CORTÉS, Discontinuous Dynamical Systems - A tutorial on notions of solutions, nonsmooth analysis, and stability, January,2007.

[13] J. ÉCALLE, Introduction aux fonctions analysables et preuve constructive de la conjecture de Dulac. Paris: Actualités Math. Hermann, 1992.

[14] A. F. FiLippov, Differential equations with discontinuous righthand side. Mathematics and Its Applications, Kluwer Academic Publishers, Dordrecht, 1988.

[15] I.S. Gradshteyn, I.M. Ryshik, Table of Integrals, Series and Products Edited by A. Jeffrey. Academic Press, New York, $5^{\text {th }}$ edition, 1994.

[16] C. HenRY, Differential equations with discontinuous righthand side for planning procedure, J. Econom. Theory 4 (1972), 541-551.

[17] D. Hilbert, Mathematical problems. Reprinted from Bull. Amer. Math. Soc. 8 (1902), 437-479. Bull. Amer. Math. Soc. (N.S.) 37 (2000), 407-436.

[18] D. Hilbert, Mathematische probleme. In Nachr. Ges. Wiss., editor, Second Internat. Congress Math. Paris, 1900, Göttingen Math.-Phys. Kl. (1900), 253-297.

[19] Y. IL'Yashenko, Finiteness theorem for limit cycles, Trans. of Math. Monogr. Am. Math. Soc. 94 (1991).

[20] T. Iто, A Filippov solution of a system of differential equations with discontinuous right-hand sides, Economic Letters 4 (1979), 349-354.

[21] N. N. Krasovskil. Stability of motion. Applications of Lyapunov's second method to differential systems and equations with delay, Stanford, CA: Stanford University Press, 1963. Translated from Russian by J. L. Brenner.

[22] I.S. KukLES, Sur quelques cas de distinction entre un foyer et un centre, Dokl. Akad. Nauk SSSR 43 (1944), 208-211. 
[23] J. Llibre, A. C. Mereu, Limit cycles for generalized Kukles polynomial differential systems. Nonlinear Analysis 74 (2011), 1261-1271.

[24] J. Llibre, A. C. Mereu, Limit cycles for a class of discontinuous generalized Lienard polynomial differential equations. Electron. J. Differential Equations. 195 (2013), 8pp.

[25] J. Llibre, A. C. Mereu, Limit cycles for discontinuous quadratic differential systems with two zones, J. Math. Anal. Appl. 413 (2014), 763-775.

[26] J. Llibre, D. Novaes, On the periodic solutions of discontinous piecewise differential systems, Preprint 2014.

[27] J. Llibre, D. Novaes, M. A. Teixeira, Higher order averaging theory for finding periodic solutions via Brouwer degree, Nonlinearity 27 (2014), 563-583.

[28] J. Llibre, D. Novaes, M.A.Teixeira, On the birth of limit cycles for non-smooth dynamical systems, to appear in Bull. Sci. Math.

[29] J. Llibre, G. Rodríguez, Configuration of limit cycles and planar polynomial vector fields, J. Differential Equations 198, (2004), 374-380.

[30] J. Llibre, G. Świrzcz, On the limit cycles of polynomial vector fields Dyn. Contin. Discrete Impuls. Syst. Ser. A Math. Anal.18 (2011), 203-214.

[31] J. Murdock, J. A. Sanders, F. Verhulst, Averaging methods in nonlinear dynamical systems. 2. ed. New York: Springer, 2007.

[32] B. E. PAden, S. S. SAStry, A Calculus for computing Filippov's differential inclucion with application to the variable structure control of robot manipulators IEEE Transactions on Circuits and Systrms 34(1987), no 1, 73-82.

[33] L. PERKo, Differential Equations and Dynamical Systems, 2nd. ed., Springer-Verlag, 1996.

[34] A. C .Rezende, Dois métodos para a investigação de ciclos limites que bifurcam de centros. Dissertação (Mestrado em Matemática), ICMC/Usp, (2011). 
[35] J. A. SAnders, F. Verhulst, Averaging methods in nonlinear dynamical systems. Applied Mathematical Sciences, 59. Springer-Verlag, New York, 1985.

[36] J. Sotomayor, Lições de equações diferenciais ordinárias . Projeto Euclides. Rio de Janeiro: IMPA, 1979.

[37] J. Sotomayor, M. A. TeixeIRA, Regularization of Discontinuous Vector Field, International Conference on Differential Equation, Lisboa, 1995, World Sci. Publ., River Edge, NJ, 1998,pp 207-223.

[38] F. Verhulst, Nonlinear differential equations and dynamical systems. Berlin: Springer-Verlag, 1989. 